Published in final edited form as:

Prog Retin Eye Res. 2008 July ; 27(4): 331-371. doi:10.1016/j.preteyeres.2008.05.001.

\title{
Vascular Endothelial Growth Factor in Eye Disease
}

\author{
J.S. Penn ${ }^{\mathrm{a}}$, A. Madan ${ }^{\mathrm{b}}$, R.B. Caldwell ${ }^{\mathrm{c}}$, M. Bartoli ${ }^{\mathrm{c}}$, R.W. Caldwell ${ }^{\mathrm{c}}$, and M.E. Hartnett ${ }^{\mathrm{d}}$ \\ aVanderbilt University School of Medicine, Nashville, TN \\ bStanford University School of Medicine, Palo Alto, CA \\ 'Medical College of Georgia, Augusta, GA \\ dUniversity of North Carolina School of Medicine, Chapel Hill, NC
}

\begin{abstract}
Collectively, angiogenic ocular conditions represent the leading cause of irreversible vision loss in developed countries. In the U.S., for example, retinopathy of prematurity, diabetic retinopathy and age-related macular degeneration are the principal causes of blindness in the infant, working age and elderly populations, respectively. Evidence suggests that vascular endothelial growth factor (VEGF), a $40 \mathrm{kDa}$ dimeric glycoprotein, promotes angiogenesis in each of these conditions, making it a highly significant therapeutic target. However, VEGF is pleiotropic, affecting a broad spectrum of endothelial, neuronal and glial behaviors, and confounding the validity of anti-VEGF strategies, particularly under chronic disease conditions. In fact, among other functions VEGF can influence cell proliferation, cell migration, proteolysis, cell survival and vessel permeability in a wide variety of biological contexts. This article will describe the roles played by VEGF in the pathogenesis of retinopathy of prematurity, diabetic retinopathy and age-related macular degeneration. The potential disadvantages of inhibiting VEGF will be discussed, as will the rationales for targeting other VEGF-related modulators of angiogenesis.
\end{abstract}

\section{Keywords}

retina; angiogenesis; vascular endothelial growth factor; age-related macular degeneration; diabetic retinopathy; retinopathy of prematurity

\section{Introduction}

\subsection{Vascular Endothelial Growth Factor (VEGF)}

Vascular endothelial growth factor (VEGF), a dimeric glycoprotein of approximately 40 $\mathrm{kDa}$, is a potent, endothelial cell mitogen that stimulates proliferation, migration and tube formation leading to angiogenic growth of new blood vessels. It is essential for angiogenesis during development; the deletion of a single allele arrests angiogenesis and causes embryonic lethality (Ferrara et al., 1996). In mammals, the VEGF family consists of seven members: VEGF-A (typically, and hereafter, referred to as VEGF), VEGF-B, VEGF-C, VEGF-D, VEGF-E, VEGF-F and PIGF (placental growth factor) (Fig. 1A). Alternative

\section{(C) 2008 Elsevier Ltd. All rights reserved.}

Corresponding author: John S. Penn, Ph.D., Vanderbilt Eye Institute, 8016 Medical Center East, North Tower, Vanderbilt University School of Medicine, Nashville, TN 37232-8808.

Publisher's Disclaimer: This is a PDF file of an unedited manuscript that has been accepted for publication. As a service to our customers we are providing this early version of the manuscript. The manuscript will undergo copyediting, typesetting, and review of the resulting proof before it is published in its final citable form. Please note that during the production process errors may be discovered which could affect the content, and all legal disclaimers that apply to the journal pertain. 
splicing results in several VEGF variants. In humans, these include the relatively abundant $\mathrm{VEGF}_{121}, \mathrm{VEGF}_{165}, \mathrm{VEGF}_{189}$ and $\mathrm{VEGF}_{206}$, and several less abundant forms (Fig. 1B). The solubility of these splice variants (collectively referred to as $\mathrm{VEGF}_{\mathrm{XXX}}$ ) is dependent on heparin binding affinity. $\mathrm{VEGF}_{206}$ and $\mathrm{VEGF}_{189}$ bind very tightly to heparin and, thus, remain sequestered in the extracellular matrix. $\mathrm{VEGF}_{165}$ binds heparin with less affinity, but also can be associated with the matrix, and $\mathrm{VEGF}_{121}$ lacks heparin-binding capacity, rendering it highly soluble. Investigations in mice genetically engineered to express less than the full complement of splice variants confirm that the relative solubility of VEGF splice variants strongly affects their specific bioactivities (Takahashi and Shibuya, 2005).

Moreover, plasmin and various metalloproteinases can cleave $\mathrm{VEGF}_{165}$, resulting in an Nterminal 113-amino acid peptide that is non-heparin-binding, but retains its bioactivity (Keyt et al., 1996; Ferrara et al., 2003). Our understanding of the relative expression of the different VEGF isoforms under normal or pathological conditions and the molecular regulators of VEGF alternative splicing is relatively limited.

Recently, discovery of the so called "VEGF $\mathrm{XXXb}_{\mathrm{X}}$ isoforms" has sparked new interest in the molecular events that regulate VEGF expression (for review, see Ladomery et al., 2007). The $\mathrm{VEGF}_{\mathrm{XXXb}}$ isoforms share approximately $94-98 \%$ homology with the corresponding $\mathrm{VEGF}_{\mathrm{XXX}}$ isoforms and have the same length. However, due to alterations in the Cterminus they bind to VEGF receptors, but do not fully activate them and act as "dominant negative splice variants" (Bates et al., 2002). Studies showing that $\mathrm{VEGF}_{165 \mathrm{~b}}$ is downregulated in angiogenic tissues (Ladomery et al., 2007) suggest a primary role for this isoform in controlling VEGF activity in health and disease. Administration of VEGF ${ }_{165 \mathrm{~b}}$ has been shown to inhibit retinal angiogenesis in the mouse model of oxygen-induced retinopathy (Konopatskaya et al., 2006).

\subsection{VEGF Receptors}

VEGFR-1/Flt-1 (fms-like tyrosine kinase) and VEGFR-2/KDR/Flk-1 (kinase insert domaincontaining receptor/fetal liver kinase), along with structurally related receptors, Flt-3/Flk-2 and VEGFR-3/Flt-4, belong to the receptor tyrosine kinase family (Fig. 1A) (Hanks and Quinn, 1991; Blume-Jensen and Hunter, 2001). VEGFR-1 and -2 are primarily involved in angiogenesis, (Yancopoulos et al., 2000) whereas Flt-3 and Flt-4 are involved in hematopoiesis and lymphangiogenesis (Jussila and Alitalo, 2000). The VEGFRs contain an approximately 750-amino-acid-residue extracellular domain, which is organized into seven immunoglobulin-like folds. Adjacent to the extracellular domain is a single transmembrane region, followed by a juxtamembrane domain, a split tyrosine-kinase domain that is interrupted by a 70 -amino-acid kinase insert, and a C-terminal tail.

VEGF receptor activation requires dimerization. Guided by the binding properties of the ligands, VEGFRs form both homodimers and heterodimers (Rahimi, 2006). The signal transduction properties of the VEGFR heterodimers, compared with homodimers, remain to be fully elucidated. Dimerization of VEGFR is accompanied by activation of receptor kinase activity, leading to autophosphorylation. Site-directed mutagenesis studies have demonstrated that the Tyr1214 residue, located in the carboxy terminus of VEGFR-2, is required for the ligand-dependent autophosphorylation of the receptor and its ability to activate signaling proteins. Signal transduction is propagated when activated VEGF receptors phosphorylate $\mathrm{SH} 2$ domain-containing protein substrates.

In addition to VEGFRs, VEGF serves as a ligand to another family of receptors, the neuropilins. Neuropilins are 120- to 130-kDa non-tyrosine kinase receptors that mediate critical functions in tumor cells and the nervous and vascular systems. In endothelial cells, neuropilins serve as receptors for the class 3 semaphorins and co-receptors for VEGF family members. The role of Neuropilin-1 (NRP-1) in mediating VEGF activity is now being 
elucidated. VEGF signaling through NRP-1 stimulates endothelial cell migration and adhesion. The addition of an anti-NRP-1 antibody suppressed the mitogenic effects of VEGF $_{165}$ on bovine retinal endothelial cells (RECs) (Oh et al., 2002). In another in vitro model, the VEGF-dependent differentiation of a subset of human bone marrow-derived cells into vascular precursors, and subsequent proliferation of these cells, required the activation of a VEGFR-2/NRP-1-dependent signaling pathway (Fons et al., 2004). Finally, VEGF promotion of the synthesis and release of prostacyclin $\left(\mathrm{PGI}_{2}\right)$, an important mediator of angiogenesis, is thought to be mediated via NRP-1 binding (Neagoe et al., 2005). The angiogenic effects regulated through VEGF binding to NRP-2 are less well characterized and appear to be modulated differently from the effects controlled by NRP-1. For example, VEGF selectively up-regulates NRP-1, but not NRP-2, on endothelial cells (Oh et al., 2002).

BIAcore analysis has shown NRP-1 to interact with VEGFR-1, greatly reducing its binding affinity for VEGF 165 (Fuh et al., 2000). Co-culture systems of endothelial cells and breast carcinoma cells indicate that NRP-1 significantly enhances VEGF 165 binding to VEGFR-2 (Soker et al., 2002). In aortic endothelial cells, NRP-2 interacted with VEGFR-1, but less is known at present about how this influences VEGF bioactivity (Gluzman-Poltorak et al., 2001). Finally, using multiple in vitro systems, NRP-2 was shown to interact with VEGFR-3, leading to lymphangiogenic activity, but no interaction was seen between NRP-2 and VEGFR-2 (Karpanen et al., 2006).

\subsection{VEGF Signaling}

Few SH2 domain-containing proteins have been shown to interact directly with VEGFR-2. Phospholipase C- $\gamma$ (PLC $\gamma$ ) binds to phosphorylated Tyr1175 (Tyr1173 in the mouse), and mediates the activation of the mitogen-activated protein kinase (MAPK) cascade, leading to proliferation of endothelial cells (Takahashi et al., 2001) (Fig. 2). PLC $\gamma$ activates protein kinase $\mathrm{C}$ via the production of diacylglycerol and increased concentrations of intracellular calcium. A Tyr1173Phe mutation of VEGFR-2 causes embryonic lethality due to vascular defects, mimicking the defects of VEGFR-2 ${ }^{-/}$mice (Sakurai et al., 2005). These data demonstrate an essential function of the Tyr1173 residue during vascular development.

In addition to PLC $\gamma$, the adaptor molecule, Shb, also binds to phosphorylated Tyr1175. VEGF-induced migration and PI3K activation is inhibited by small interfering RNA (siRNA)-mediated knockdown of Shb in endothelial cells (Holmqvist et al., 2004). The serine/threonine kinase, Akt, is activated downstream of PI3K and mediates endothelial cell survival (Fujio and Walsh, 1999). Akt also regulates nitric oxide (NO) production by direct phosphorylation and activation of endothelial NO synthase (eNOS). Finally, phosphorylated Tyr1175 is known to interact with Sck (Igarashi et al., 1998; Sakai et al., 2000), an adaptor molecule that binds Grb2, and participates in MAPK signaling in the epidermal growth factor pathway (Thelemann et al., 2005).

Another important phosphorylation site in VEGFR-2 is Tyr951 (Tyr949 in the mouse), a binding site for the signaling adaptor VRAP (VEGF receptor-associated protein) (Matsumoto et al., 2005). The phosphorylated Tyr951-VRAP pathway has been shown to regulate endothelial cell migration (Matsumoto et al., 2005; Zeng et al., 2001). Reduced microvessel density and tumor growth in $\mathrm{VRAP}^{-/-}$mice confirm an essential function for this residue in endothelial cells of the angiogenic phenotype (Matsumoto et al., 2005). VEGF induces the formation of a complex between VRAP and Src (Matsumoto et al., 2005), indicating that VRAP might regulate Src activation and its signaling downstream of VEGFR-2.

Mice that express a Tyr1212Phe (corresponding to the human Tyr1214) VEGFR-2 mutant are viable and fertile (Sakurai et al., 2005). However, phosphorylation of Tyr1212/1214 has 
been implicated in VEGF-induced actin remodeling through the sequential activation of CDC42 and p38 MAPK (Lamalice et al., 2004). Inhibition of p38 MAPK augments VEGFinduced angiogenesis in the chicken chorioallantoic membrane (CAM) assay (Issbrucker et al., 2003; Matsumoto et al., 2002), without an accompanying increase in vascular permeability (Issbrucker et al., 2003). Moreover, p38 MAPK induces phosphorylation of heat-shock protein-27 (HSP27), a molecular chaperone that positively regulates VEGFinduced actin reorganization and migration (McMullen et al., 2005; Rousseau et al., 1997).

The existence of multiple ligands and receptors provides initial diversity to VEGF bioactivity. Groupings of receptor homo- and heterodimers, activated by both common and specific ligands, further augment the diversity of VEGF signaling. A final level of diversity is provided by activation of distinct signaling intermediates downstream of each VEGF receptor. The combination of these features yields an elaborate signaling network capable of regulating the extremely complex angiogenic cascade.

\subsection{Regulation of VEGF Expression}

During hypoxia, VEGF gene expression increases via several different mechanisms (Dor et al., 2001). These mechanisms include increased transcription, mRNA stability, and protein translation using an internal ribosomal entry site, as well as increased expression of oxygen regulated protein 150, a chaperone required for intracellular transport of proteins from the endoplasmic reticulum to the Golgi apparatus prior to secretion (Chen and Shyu, 1995; Forsythe et al., 1996; Levy et al., 1996; Levy et al., 1998; Ozawa et al., 2001).

The increase in VEGF transcription is largely mediated via hypoxia inducible factor-1 (HIF-1) (Fig. 3). HIF-1 is a heterodimeric transcription factor composed of two subunits the constitutively produced HIF- $1 \beta$ subunit and the inducible component, HIF-1a (Wang and Semenza, 1995). Under normoxic conditions, HIF-1a is inactivated and targeted for proteasomal degradation by hydroxylation, whereas under hypoxic conditions the specific hydroxylases are inhibited, resulting in the rescue of HIF-1a from degradation (Schofield and Ratcliffe, 2004). When this occurs, HIF-1a complexes with HIF-1 $\beta$, translocates into the nucleus and binds to a specific sequence in the $5^{\prime}$ flanking region of the VEGF gene, the hypoxia responsive element (HRE) (Ikeda et al., 1995; Laughner et al., 2001; Shima et al., 1996; Wenger, 2002; Forsythe et al., 1996). The importance of interaction between HIF-1a and the VEGF promoter has been confirmed in studies of HIF-1 $\mathrm{a}^{-/-}$mouse embryonic stem cells, in which basal expression of VEGF mRNA remains low in response to hypoxia (Carmeliet et al., 1998; Iyer et al., 1998).

Two additional isoforms of HIF, known as HIF- $2 a$ and HIF-3 $a$, have been identified by screening for proteins that associate with HIF-1 $\beta$ (Ratcliffe, 2007). HIF-2 $\alpha$ appears to be closely related to HIF-1 $a$ and can promote HRE-dependent gene transcription. While structurally and functionally similar, HIF- $1 a$ and HIF- $2 a$ appear to exert different biological functions, as demonstrated in studies using knockout mice (Hu et al., 2003). For example, while HIF-1a antagonizes c-Myc function, inhibiting renal cell carcinoma (RCC) growth, HIF-2a promotes cell cycle progression in hypoxic RCC and many other cell lines (Gordan et al., 2007). Interestingly, the most distantly related isoform, HIF-3a, appears to antagonize HRE-dependent gene expression, suggesting a possible negative influence on hypoxiainduced gene expression. Additional study is needed to determine if HIF-2a or HIF- $3 a$ is involved in the regulation of retinal VEGF expression.

Clearly, post-transcriptional events are also important in the regulation of VEGF production in the diseased retina, as underscored by the correlation of polymorphisms within the $5^{\prime}$ untranslated region (UTR) of the VEGF gene with the occurrence of age-related macular degeneration (AMD). The $3^{\prime}$ UTR and the $5^{\prime}$ UTR of the VEGF gene are important sites of 
regulation controlling mRNA stability and the rate of translation through the internal ribosomal entry site (IRES) (for review, see Yoo et al., 2006).

Evidence for the increase in VEGF mRNA stability in response to hypoxia comes from in vitro mRNA degradation assays that have led to the identification of adenylate/uridylate-rich elements (AREs) in the 3' UTR of VEGF mRNA. VEGF mRNA is extremely labile in normoxic conditions, with a half-life of less than 1 hour, as compared with the average halflife of 10 to 12 hours for eukaryotic mRNA. During hypoxia, the half-life of VEGF mRNA increases by two to three-fold (Levy et al., 1996) due to a stabilizing effect of HuR, a 36 $\mathrm{kDa}$ RNA-binding protein, which binds with high affinity to AREs in the $3^{\prime}$-UTR of VEGF mRNA, protecting it from degradation by endonucleases (Brennan and Steitz, 2001; Robinow et al., 1988).

Post-transcriptional regulation can also occur in the in $5^{\prime}$-UTR of VEGF mRNA. This region contains multiple IRES. These are specific sites of attachment to the ribosomal machinery, which provide sites for initiation of translation alternative to the classical $5^{\prime}$ capand elF-dependent translational system (for review, see van der Velden and Thomas, 1999). Several IRES have been identified in the $5^{\prime}$-UTR of VEGF mRNA, and these provide alternative sites of translational control of VEGF expression (Bornes et al., 2004). Notably, evidence suggests that IRES sites in the VEGF $5^{\prime}$-UTR can potentially control the generation of alternatively spliced VEGF (Bornes et al., 2004; Huez et al., 2001).

Another regulatory mechanism consists of increased production of oxygen regulated protein 150 (ORP150) in response to hypoxia. Studies using human macrophages transfected with adenovirus coding for ORP150 showed that overexpression of ORP150 resulted in increased VEGF secretion in hypoxia. Evidence suggests that under hypoxic conditions, ORP150 functions as a molecular chaperone to facilitate VEGF protein transport and secretion (Ozawa et al., 2001). VEGF is not only regulated by hypoxia.

VEGF function is also affected by insulin like growth factor 1 (IGF-1) which plays an important role in retinal vascularization. Several lines of evidence, including in vitro studies, support the notion that IGF-1 is critical for vessel development (King et al., 1985; Grant et al., 1993). Preterm infants with reduced serum levels of IGF-1 have a higher incidence of development of retinopathy (Hellstrom et al., 2003). Mice null for the IGF-1 gene have retarded retinal vascular growth, compared to wild type controls (Hellstrom et al., 2001). However, the action of IGF-1 is not mediated by decreasing VEGF expression, as the amount of VEGF mRNA is similar in knock out and wild type control mice; instead IGF-1 acts by decreasing VEGF activation of the Akt signaling pathway. Both MAPK and Akt pathways have been shown to be necessary for endothelial cell survival (Smith et al., 1999).

\subsection{Retinal Expression of VEGF and VEGFR}

The influence of VEGF in retinal diseases is profound. It has been implicated in a large number of retinal diseases and conditions including, but not limited to, highly prevalent conditions like AMD and diabetic retinopathy; less common disorders such as retinopathy of prematurity, sickle cell retinopathy and retinal vascular occlusion; and as a non-causal, but important, secondary influence in neovascular glaucoma (Bock et al., 2007) and inherited retinal dystrophies (Penn et al., 2000). Collectively, these conditions, all of which have critical angiogenic components, account for the vast majority of irreversible vision loss in developed countries.

At least five retinal cell types have the capacity to produce and secrete VEGF. These include the retinal pigmented epithelium (RPE) (Miller et al., 1997), astrocytes (Stone et al., 1995), Müller cells (Robbins et al., 1997), vascular endothelium (Aiello et al., 1995) and ganglion 
cells (Ida et al., 2003). These cells differ widely in their responses to hypoxia; in vitro studies show that Müller cells and astrocytes generally produce the greatest amounts of VEGF under hypoxic conditions (Morrison et al., 2007; Aiello et al., 1995; Hata et al., 1995). To date, the relative capacity of these cells to produce specific splice variants remains unclear, as do the patterns of splice variant production throughout retinal development and aging.

The distinct roles of the different VEGF splice variants in retinal vascular development is being explored, however, in mice expressing only a single variant (Stalmans et al., 2002). Vascular development was normal in the retinas of mice expressing only $\mathrm{VEGF}_{164}$ $\left(V E G F^{164 / 164}\right.$ ), indicating that this variant is sufficient for directing normal vascular growth and remodeling. In contrast, retinas of $V E G F^{120 / 120}$ mice exhibited severe vascular defects, displaying retarded venous and severely flawed arterial development. $V E G F^{188 / 188}$ mice had normal development of retinal veins but little or no arterial growth.

Evidence for the expression patterns and roles of VEGFR in retinal tissues comes from a variety of species and experimental venues. In the human retina VEGFR-1 and -2 can be expressed by neural, glial and vascular elements. In adults, expression is generally restricted to the inner nuclear layer (Müller cells and amacrine cells), the ganglion cell layer, and the retinal vasculature (Stitt et al., 1998). However, during retinal neurogenesis VEGFR-2 is also expressed by neural progenitor cells (Hashimoto et al., 2006). Notably, neural cell VEGFR-2 can be activated by VEGF in vitro (Yang et al., 1996). In cultured retinal pericytes VEGFR-1, but not -2, is expressed (Takagi et al., 1996), whereas in cultured RPE cells, both receptors are expressed and are induced by oxidative stress (Sreekumar at al., 2006). In the mouse, ganglion cells express both receptors, but only VEGFR-2 is increased by intraocular inoculation with herpesvirus (Vinores et al., 2001). Studies in newborn mice using the VEGFR-specific kinase inhibitor, SU5416, indicate that Müller cell survival or proliferation during retinal development is VEGFR- and MAPK-dependent (Robinson et al., 2001). In a study of patients with diabetic retinopathy, VEGFR-1 expression dominated in normal retina, but was not increased in the diabetic retina, while VEGFR-2 levels were increased, particularly in the vascular elements (Smith et al., 1999). Finally, VEGFR-1 and -2 are found on uterine smooth muscle cells in vivo. When these cells are cultured in vitro, VEGFR-1 can be phosphorylated and is capable of inducing smooth muscle cell proliferation (Brown et al., 1997). To date, neither VEGFR-1 nor -2 has been identified in retinal smooth muscle cells.

This article will review the role of VEGF in angiogenesis related to three blinding conditions: retinopathy of prematurity, diabetic retinopathy and age-related macular degeneration. These conditions constitute the leading causes of irreversible vision loss in infants, and working age and elderly Americans, respectively. That VEGF is believed to play a causal role in all three of these disorders underscores its profound impact in eye disease. VEGF antagonists have already proven their value in tumor angiogenesis and choroidal neovascularization, and new VEGF antagonists are being tested pre-clinically and clinically for other ocular indications. Only with a more complete understanding of VEGF and its retinal and choroidal activities can we hope to develop better strategies to prevent, retard or repair the damage caused by ocular neovascularization.

\section{Retinopathy of Prematurity}

Retinopathy of prematurity (ROP), a neovascularizing disease affecting preterm infants, is one of the most common causes of childhood blindness in the world. Recent estimates indicate that each year in the United States, 68\% of the approximately 10,000 babies born with a birth weight of less than 1250 grams will develop ROP. Thirty six percent of these 
infants will progress to severe ROP, a condition that can lead to retinal detachment and blindness. The incidence of the disease is highly correlated with the gestational age of the infant at birth. That is, the more immature the infant, the higher the likelihood of developing severe ROP (CRYO-ROP Cooperative Group, 1988; ETROP Cooperative Group, 2003). ROP is caused by perturbation of the process of normal vascular development of the retina. Its correlation with gestational age stems from the fact that the retina is one of the last organs to be vascularized in the human fetus. In very premature infants, the retina is nearly avascular at birth. The process of retinal vascular development normally occurs in the hypoxic uterine environment, but it must occur in a relatively hyperoxic extra-uterine environment in these infants. For reasons defined in the following paragraphs, this leads to arrested growth of retinal blood vessels, followed by their unregulated growth into the vitreous cavity, with potentially catastrophic consequences.

\subsection{Pathogenesis of ROP}

The pathogenesis of ROP is hypothesized to consist of two distinct phases (Madan and Penn, 2003). In the initial phase, normal retinal vascular growth is retarded. This occurs as a consequence, primarily, of exposure to extra-uterine hyperoxia aggravated by therapeutic oxygen delivery, but it may also be due to other noxious stimuli, and/or to the premature withdrawal of certain maternally derived factors at the time of birth. Relative retinal hypoxia results from the increasing metabolic demands of the developing neural retina that are unmet secondary to the attenuation of blood vessels. This leads to the second phase of ROP, consisting of the release of VEGF and other angiogenic factors, producing excessive growth of abnormal leaky blood vessels into the vitreous, followed by vitreous hemorrhage and tractional retinal detachment. This second phase is probably encouraged by the weaning of infants from oxygen therapy, but removal from therapy is not required for the neovascular response to occur. Understanding the process of normal retinal vascularization can provide important clues regarding the molecular mechanisms underlying the pathogenesis of the two phases of ROP.

\subsection{Development of the Retinal Vasculature}

The process of normal vascularization has been extensively examined in the kitten, mouse and rat retinas (Ashton et al., 1957; Ashton, 1961; Ashton, 1970; Blanks and Johnson, 1983; Connolly et al., 1988; Chan-Ling et al., 1990; Smith et al., 1994; Stone et al., 1995; Dorrell and Friedlander, 2006). Several studies have also examined the process in the human fetal retina (Michaelson, 1948; Nilausen, 1958; Cogan, 1963; Ashton, 1970; Nishimura and Taniguchi, 1982; Penfold et al., 1990; Gariano et al., 1994; Hughes et al., 2000).

Development of the retinal vasculature follows a common pattern in all species (Dorrell and Friedlander, 2006), but there are some dissimilarities as well (Ashton, 1968; Gariano et al., 1994). For example, in humans the process occurs primarily during the latter half of gestation, whereas in rodents the process is completed in the first two weeks after birth (Michaelson et al., 1954; Ashton, 1970; Stone et al., 1995; Engerman and Meyer, 1965). Generally, the completion of retinal vascular development is coincident with eye opening in mammalian species.

Retinal tissue is provided with oxygen and nutrients by the adjacent choriocapillary plexus, which supplies the avascular photoreceptor layers, and by the superficial and deep capillary plexuses within the retina, supplying its inner layers (Yu and Cringle, 2001). The superficial plexus lies immediately beneath the inner limiting membrane, while the deep plexus permeates the inner nuclear layer. The endothelial cells lining the vessels in the retinal capillary plexuses form tight junctions and provide an important part of the blood-retinal barrier, while the choroidal vessels are fenestrated, lacking this barrier (Raviola, 1977; Campochiaro, 2000). At the choroid/retina interface, barrier function is provided by tight 
junctions between adjacent retinal pigment epithelial cells. Additionally, prior to the formation of the retinal vasculature, a transient network of vessels called the hyaloid vascular system forms to nourish the immature lens. The vessels of the hyaloid extend from their source at the optic nerve head to the posterior surface of the lens, where they bifurcate to form the dense capillary arbor known as the tunica vasculosa lentis. This network regresses during mid-gestation in the human and two to three weeks post-natally in the mouse and rat, coinciding with the period of retinal vascular development (Gogat et al., 2004; Fruttiger, 2007).

2.2.1 The Superficial Plexus-Retinal astrocytes guide the formation of the retinal vasculature in mammals. Immediately prior to the development of the vascular plexus, a network of astrocytes radiates from the optic nerve head across the surface of the immature retina in a central to peripheral pattern. This network forms the scaffold upon which the primary vascular plexus is formed (Ling and Stone, 1988; Fruttiger et al.,1996). Astrocytes express VEGF, which promotes endothelial cell proliferation and migration within this superficial plane (Stone et al., 1995). The formation of this primary vascular plexus begins in the region around the optic disc at the base of the hyaloid artery. A network of capillaries spreads across the developing neural layer along the inner surface of the retina towards the periphery. Because of its location, this vessel network is often called the superficial plexus. In rodents, the development of the superficial plexus begins at birth, while in humans the process begins at about 16 weeks gestation (Provis, 2001; Saint-Geniez and D'Amore, 2004; Dorrell and Friedlander, 2006).

VEGF expression in the retina is closely linked to retinal vascular development. On postnatal day zero (P0), intense VEGF expression is noted in the innermost nerve fiber layer in the mouse retina. From P0 - P7, expression increases and is greater at the leading edge of blood vessel growth (Gariano et al., 2006). As blood vessel growth proceeds, there is a gradual decrease in VEGF expression (Fig. 4). The pattern suggests a strong correlation between the presence or absence of physiologically patent blood vessels and the level of local VEGF expression.

Studies indicate that development of the retinal vasculature may occur by both vasculogenesis, de novo formation of blood vessels from mesodermal precursor cells (angioblasts), and angiogenesis, sprouting of new vessels from existing blood vessels (Ashton, 1966; Ashton, 1970; Flower et al., 1985; McLeod et al., 1987; Kretzer and Hittner, 1988; Chan-Ling et al., 1990; Jiang et al., 1995; Risau and Flamme, 1995; Risau, 1997; Hughes et al., 2000). "Spindle-cells", so called for their shape, have been identified within the inner plexiform layer of the immature mammalian retina (Flower et al., 1985; McLeod et al., 1987; Stone and Dreher, 1987; Watanabe and Raff, 1988; Hughes et al., 2000; Taomoto et al., 2000). These cells are purported to coalesce to form vascular cords, which subsequently form the patent vessels that constitute the superficial vascular plexus. By a process that is not yet completely understood, the vessels then undergo remodeling, leading to the development of mature arteries, veins and capillaries. The identification of vascular precursor cells in the retinas of various species supports the notion that vasculogenesis participates in the formation of the primary plexus (Chan Ling et al., 2004). However, some evidence argues in favor of angiogenesis as being the mechanism driving formation of the primary plexus (Gariano et al., 1996; Provis, 2001; Fruttiger, 2002; Rehman et al., 2003). The controversy appears to be centered around the methods used to establish the presence of the precursor cells (Chan-Ling et al., 2004; McCleod and Hasegawa, 2006; Urbich and Dimmeler, 2004). Some investigators have used non-specific ADPase or Nissl staining to identify vascular precursor cells (Hughes et al., 2000; Lutty and McLeod, 1992), but the absence of specific markers of endothelium, such as VEGFR-2 and CD31 (also known as PECAM-1 for platelet endothelial cell adhesion molecule), in these cells has caused other 
investigators to question whether they are vascular precursors, astrocyte precursors or microglia (Fruttiger, 2002; Provis and Sandercoe, 2000). Still other investigators argue for combined contributions from both angiogenesis and vasculogenesis in the development of the primary vessel plexus. Their findings indicate that, in addition to clearly defined endothelial cells, specific subpopulations of hematopoietic stem cells (HSCs) can selectively target the retinal astrocyte template and participate in the formation of a stable retinal vasculature (Dorrell et al., 2002). Unlike spindle cells, which are believed to migrate through the retina from the optic nerve head, these stem cells would presumably be delivered to specific sites of retinal vessel growth by the circulation.

Studies have cited VEGFR-2 transcripts, identified in cells in the avascular human fetal retina, as providing evidence for the presence of vascular precursor cells in the primordial plexus of the human retina (Chan-Ling et al., 2004; Gogat et al., 2004). However, this argument is confounded by the expression of VEGFR-2 by a variety of non-vascular cell types (Ferrara et al., 2003; Otrock et al., 2007). It is possible that there may be speciesspecific differences in the process of retinal vascularization. Further work is needed to confirm the mechanism of formation of these early retinal blood vessels; the answers will have important implications for the understanding and prevention of ROP.

There is a close correlation between migration of retinal astrocytes, expression of VEGF and development of the superficial vascular plexus; the avascular fovea in humans does not contain astrocytes (Chan-Ling et al., 1992; Holash and Stewart, 1993; Gariano et al., 1994; Jiang et al., 1994; Sandercoe et al., 1999; Dorrell et al., 2002; Fruttiger, 2007). Initially, superficial blood vessels follow the central to peripheral gradient of retinal astrocyte maturation towards the periphery of the retina, which is reached by P8 in mice, P12 in rats and 32 weeks gestation in the human. Yet, while there are substantial differences in its time of onset and completion, the spatial pattern of vascular development in the human retina differs only slightly from that in rodents and the cat, in that the vessels do not always strictly follow the astrocyte scaffolding throughout the retina (Provis et al., 1997). Such interspecies correlations are notable, because formation of the human retinal vasculature occurs in utero, where arterial oxygen tension is $30 \mathrm{~mm} \mathrm{Hg}$ or less, while the cat and rat retinal vasculatures are formed after birth, at substantially higher arterial oxygen tensions. Under these disparate circumstances, the controlling molecular influences in the two cases could differ markedly.

Those who believe that the primordial vessel network develops via an angiogenic mechanism argue that the process is largely governed by VEGF. As the network gradually covers the retina, the increased oxygen delivery to the retinal tissue triggers a negative feedback loop, decreasing VEGF expression and endothelial cell proliferation, while increasing astrocyte differentiation (West et al., 2005). Alternatively, those who believe that vasculogenic growth drives the formation of the primary network, contend that the process is not dependent on metabolic demand and physiologic hypoxia (Hughes et al., 2000). Their case is supported by several observations: 1) VEGF expression is not detected in the inner layers of the human retina until after the time when active retinal vasculogenesis has been observed (Provis et al., 1997); 2) vasculogenesis is established at about 15 weeks gestation, a period prior to active neuronal differentiation (Provis et al., 1985) and although neuronal maturation is highest in the perifoveal region during this period, this region is vascularized much later in gestation; and 3) although VEGF null mice are not viable and have abnormal blood vessels, vessel formation does occur in these animals (Carmeliet et al., 1996).

2.2.2 The Deep Plexus-Following behind the progression of the superficial plexus to the retinal periphery, another wave of new blood vessels forms. These vessels sprout from the superficial plexus and extend into the deeper layers of the retina, forming the deep 
vascular plexus. CD34 staining, confirmatory for endothelial cells, demonstrates that the increase in vascular density of the superficial plexus and the growth of the deep plexuses occur by angiogenesis (Hughes et al., 2000; Fruttiger, 2007). In the human fetus, this process begins around 25 weeks gestation, simultaneous with the appearance of the visual evoked potential (Leaf et al., 1995; Hughes et al., 2000; Dreher and Robinson, 1998;). The role of hypoxia-induced VEGF expression in this angiogenic process is widely accepted. The "physiologic hypoxia" created by the increasing metabolic demands of the developing neural components of a largely avascular retina is the driving mechanism for this angiogenic growth. Low tissue $\mathrm{PO}_{2}$, hypoxia, results in secretion of retinal VEGF, and this leads to additional elaboration of the superficial vessel network and development of the deep network (Chan-Ling et al., 1990; Chan-Ling et al., 1995b; Stone et al., 1995; Zhang and Stone, 1997; Salhia et al., 2000). It is interesting to speculate that, because of their spatial distributions, astrocytes control the final growth phase and refinement of the superficial capillary network, while Müller cells drive growth of the deep network. In any case, increased blood vessel growth and capillary density relieves hypoxia, thus matching vessel formation to tissue oxygen demand. This hypothesis, however, still does not explain the lack of blood vessels in the fovea, a region with high metabolic activity. It is possible that the lack of retinal astrocytes in this region, and the subsequent lack of VEGF expression, may be responsible for the avascularity (Hughes et al., 2000), or it may be that an as yet unidentified angiostatic factor discourages vessel growth in this retinal region.

Retinal vessels extend toward the sclera until the interface of the inner nuclear layer and the photoreceptor layers is reached, where the "deepest" vessel layer develops (Hughes et al., 2000; Provis, 2001; Fruttiger, 2002; Gariano, 2003). This is followed by formation of an intermediate vessel layer at the vitread edge of the inner nuclear layer. These two layers of vessels, bordering the inner nuclear layer on its distal and proximal surfaces, constitute the deep plexus (Fig. 5). Vascular growth in the perifoveal and temporal retinal regions, like that of the deep plexus, occurs by angiogenesis alone (Connolly et al., 1988; Stone et al., 1995; Hughes et al., 2000). In contrast to the formation of the primary plexus, development of the deep plexus is independent of retinal astrocytes.

Again, vascular growth is closely regulated by supply and demand of oxygen. High oxygen tension suppresses hypoxia-induced VEGF production, and less VEGF results in less blood vessel growth. With development of the capillary plexuses and the resulting increase in oxygen tension, a capillary-free zone develops around the major blood vessels, followed by vessel retraction in the superficial plexus. This process is particularly evident around the oxygen-rich arteries, where, in the rat and kitten retinas, the diameter of the capillary-free zone is dependent on the animal's ambient oxygen environment (Michaelson, 1948; Campbell, 1951; Phelps, 1990) (Fig. 6). Interestingly, vascular pruning in the developing retina does not result from apoptosis, but from endothelial cell migration from retracting vessels into the surrounding newly developing vessels (Hughes et al., 2000). The process of natural pruning can be accelerated by experimental exposure to hyperoxia. And, although VEGF stimulates endothelial cell migration, administration of VEGF can prevent vessel regression under hyperoxic conditions, indicating that its role in vessel regression during normal retinal vascular development is complex and potentially paradoxical (Alon et al., 1995).

Maturation of the newly developed vascular plexus occurs when pericytes are recruited to the endothelial plexus. Pericyte recruitment, which lags behind the formation of the vascular plexus, involves the migration of pericytes in an arteriole-to-venule direction, progressively, until all of the vessels are ensheathed by pericytes. This occurs by three weeks of age in the rodent retina (Benjamin and Hemo, 1998). The differentiation of immature mesenchymal cells into pericytes upon contact with endothelial cells is associated with increased VEGF 
expression. VEGF produced by differentiated pericytes plays an important role in pericyte spreading over endothelial cells in both normoxic and hyperoxic conditions (Darland and Massingham, 2003; Benjamin and Hemo, 1998). Other factors, including PDGF- $\beta$ and TGF$\beta$ secreted by endothelial cells, and the angiopoietins also play a role in pericyte recruitment (Lindahl et al., 1997; Hirschi and D'Amore, 1996; Suri et al., 1996). Mature vessels covered with pericytes appear less responsive to VEGF (Benjamin and Hemo, 1998; Alon et al., 1995). This may explain the extreme vulnerability of the premature infant retina to hyperoxia, in contrast to the more mature infant retina.

\subsection{VEGF and Retinal Vascular Development}

VEGF has been identified as the key factor driving the development and growth of blood vessels (Ferrara and Davis-Smyth, 1997). New vessel growth is a complex process requiring activation of several receptors and growth factors. Of these growth factors, VEGF is essential for proper physiological angiogenesis (Ferrara, 2004). Increased VEGF expression is seen in tissues undergoing angiogenesis, and an increase in VEGF receptors is seen on target endothelial cells in the vicinity (Jakeman et al., 1993; Robbins et al., 1998). In the developing mouse, VEGF expression is first seen in trophoblast cells within a few days of implantation, and expression is highest in the choroid plexus and ventricular epithelium (Breier et al., 1992). Mouse embryos with disruption of one allele of the VEGF gene have defective vascularization and reduced nucleated red blood cells within blood islands, as well as developmental anomalies in the forebrain and heart outflow tract (Carmeliet et al., 1996; Ferrara et al., 1996). In addition to exerting a mitogenic influence on endothelial cells, VEGF also functions as a survival factor (Alon et al., 1995; Ferrara and Davis-Smyth, 1997; Gerber et al., 1998a, 1998b). These survival effects appear to be developmentally regulated, as inhibition of VEGF results in apoptotic changes only in the neonatal vasculature (Gerber et al., 1999).

The expression of VEGF in the developing retina has been examined in various species and in the human fetus. Evidence for the interaction between VEGF, VEGF receptors, and neuroglial cells of the retina in normal vascular development comes from several studies (Alon et al., 1995; Stone et al., 1995; Yang and Cepko, 1996; Provis et al., 1997; Robinson et al., 2001; Feeney et al., 2003; Gogat et al., 2004). In both animals and humans, VEGF expression is initially seen in the astrocytes of the neuroblastic layer adjacent to the inner limiting membrane near the optic disc, and it advances towards the periphery with a gradual down-regulation in the central retina. VEGF expression always precedes the advancing blood vessels until the vessels reach the peripheral margin of the retina (Chan-Ling et al., 1995b; Stone et al., 1995; Pierce et al., 1996; Provis et al., 1997). With advancing age, in rat and kitten retinas, VEGF expression disappears from the superficial layer, and there is a second wave of VEGF expression in the Müller cells of the inner nuclear layer. This correlates with the formation of the deep vascular plexus. Similar findings were reported in the human fetal retina with a strong spatial correlation between VEGF expression and increased neuronal differentiation and metabolic activity (Provis et al., 1997). Taken collectively, these studies demonstrate that VEGF expression in the retina is temporally and spatially related to the development of the vasculature.

The choriocapillaris develops prior to the development of the retinal vasculature (Gogat et al., 2004). VEGF mRNA in retinal pigment epithelial cells (RPE), along with Flk-1 mRNA on neighboring endothelial cells in the rat, kitten and human fetal retina suggest that VEGF plays a role in the development of the choroidal vasculature (Yi et al., 1998; Gogat et al., 2004). This expression is not related temporally or spatially to normal retinal vessel development, and VEGF expression by RPE is not down-regulated by hyperoxia (Stone et al., 1995). Vessel tone in the choroidal vasculature does not respond to hyperoxia, thus allowing for greater diffusion of oxygen under such conditions into the inner retina. 
Although the vessels of the choroid plexus likely play a role in making the immature retina susceptible to hyperoxic injury, the neovascularization or aberrant growth of blood vessels seen in ROP arises only from the superficial and deep plexuses of the retina. These vessels lose their dependence on VEGF as they mature and become associated with mural cells, again, possibly explaining the absence of their sensitivity in full-term infants (Alon et al., 1995).

\subsection{Regulation of VEGF Gene Expression by Oxygen Tension}

As discussed above, oxygen tension and VEGF play a key role in retinal blood vessel growth. VEGF gene expression is tightly regulated by oxygen tension, a unique feature that helps tissues adjust vascular supply to oxygen demand (Goldberg and Schneider, 1994). Current knowledge suggests that under normoxic conditions, VEGF is produced by retinal cells at a level adequate to support existing blood vessels. VEGF expression is decreased in response to hyperoxia, both in cultured cells and in astrocytes and Müller cells in retinas of P10 rats and P7 mice exposed to hyperoxic breathing conditions (Alon et al., 1995; Pierce et al., 1996). The decrease in VEGF expression by hyperoxia is hypothesized to play an important role in the first stage of ROP. Hyperoxic episodes, as are periodically seen in preterm infants on oxygen therapy, result in suppression of VEGF expression, increased apoptosis and vasoattentuation (Fig. 4) (Alon et al., 1995; Pierce et al., 1996). In contrast, hypoxia, an important stimulus for blood vessel growth, results in increased VEGF gene expression (Shweiki et al., 1992; Levy et al., 1995; Forsythe et al., 1996). Paracrine secretion of VEGF in response to hypoxia occurs from numerous ocular cells, including RECs, retinal pericytes, Müller cells, and astrocytes (Adamis et al., 1993; Aiello et al., 1995a; Eichler et al., 2004; Robbins et al., 1997).

In the normal mouse retina, there is a virtual absence of capillaries and VEGF mRNA expression around arteries and in the region surrounding the optic disc. Hyperoxic exposure of the mouse or rat at P7 causes an expansion of these capillary-free regions by decreasing expression of VEGF mRNA and increasing vessel attenuation (Fig. 6). It is hypothesized that the strongly attenuative effect of hyperoxia on the central vasculature in mice is the result of oxygen diffusion from arterial blood in the arteries of optic nerve region where they are close proximity to one another (Claxton and Fruttiger, 2003). Exposing P0 mice to hyperoxia for 7 days resulted in an avascular retina until at least P8 and increased expression of VEGF in the surrounding astrocytes. The explanation forwarded for the absence of an angiogenic response despite increased VEGF expression is that, at P0, the retinal blood vessels have not yet developed and therefore the effect of hyperoxia is not easily transmitted to the retinal tissue as elevated $\mathrm{PO}_{2}$. High VEGF levels in this situation are unable to trigger angiogenesis in the hypoxic retina because the region immediately around the hyaloid artery is hyperoxic from diffusion of oxygen and is capillary free, meaning that it is unable to sprout vessels into the surrounding hypoxic retina (Claxton and Fruttiger, 2003).

\subsection{VEGF and Abnormal Retinal Vascularization}

Sixty years ago, Michaelson first suggested the presence of an angiogenic factor responsible for neovascularization in retinopathy (Michaelson, 1948), but it was not until the early 1980s that VEGF was identified as the key angiogenic molecule (Senger et al., 1983; Keck et al., 1989). VEGF is a relatively endothelial cell-specific mitogen, promoting endothelial cell growth and survival (Alon et al., 1995; Ferrara and Davis-Smyth, 1997; Gerber et al., 1998a, 1998b). VEGF also causes increased vessel permeability and induction of a fenestrated phenotype, two features of VEGF bioactivity that are responsible for the increased leakiness and retinal hemorrhages seen in severe ROP (Roberts and Palade, 1995; Bates and Curry, 1997). 
Several studies in humans and animals point to the role of VEGF in retinopathy. VEGF levels are increased in ocular fluid from patients with diabetic retinopathy (Adamis et al., 1994; Aiello et al., 1994) and other retinal neovascularizing diseases (Pe'er et al., 1995). In situ hybridization analyses showed the proliferation of blood vessels to be accompanied by an induction of retinal VEGF expression only in the retinal layer effected by decreased perfusion (Pe'er et al., 1995). Also, transgenic mice with overexpression of VEGF in the photoreceptor layer develop intraretinal and subretinal neovascularization (Okamoto et al., 1997). In a single report in the literature, in situ hybridization studies of an autopsy specimen with stage 3 ROP showed increased VEGF mRNA expression in the peripheral avascular region in the ganglion cell layer and inner nuclear layer, confirming the results from the animal studies described below (Young et al., 1997).

Much of the evidence for the role of VEGF in ROP has been acquired through careful studies in animal models of oxygen-induced retinopathy (OIR) (Madan and Penn, 2003). All of the models have used hyperoxia to initiate vasoattenuation (Chan-Ling et al., 1992; Penn et al., 1993; Smith et al., 1994). The mouse model of retinopathy is one of the most commonly used models of ROP (Smith et al., 1994). In this model, P7 mice are exposed to a $75 \%$ oxygen environment for a period of 5 days to initiate vasoattenuation and are then returned to room air, which produces retinal hypoxia (Fig. 7). The vasoattenuative response in the central retina occurs within 2 days, along with loss of astrocytes from the same region (Gu et al., 2002). Neovascularization in this model is seen at P17 at the boundary between the avascular central retina and the mid-peripheral retinal vessels that are the origin of the new growth. One of the main differences between normal and pathological retinal vessel development is that abnormal neovascular tufts develop in place of normally sprouting vessels in capillary-depleted areas (Fig. 8). These tufts often lack physiologically patent lumena, meaning that they do not efficiently carry oxygen, which prevents them from addressing the hypoxia that underlies their growth and may be responsible for their persistence.

The rat model developed by Penn yields a pattern of retinopathy that is similar to that seen in the human infant suffering ROP, one of peripheral avascularity (Penn et al., 1993). Seeking to mimic the retinal tissue oxygen pattern of a newborn infant on oxygen therapy, this model employs an exposure paradigm in which the oxygen is cycled between $50 \%$ and $10 \%$ every 24 hours for the first 2 weeks of life. Neovascular tufts develop upon removal to room air in the retinal mid-periphery, at the interface of the regions of vascular and avascular retina (Fig. 9). Abnormal intraretinal vessel growth is observed late in the oxygen exposure period, while the first signs of neovascular tuft formation are seen at 2 days postexposure, and the surface of the retina is breached by the vessel tufts at approximately 4 days post-exposure.

Additional knowledge of the response of immature retinal vessels to oxygen has been obtained by studies performed in developing kittens and puppies, although much less is known about the role of VEGF in these species. The level of retinal vascular development in the newborn kitten is more advanced than that of the newborn mouse or rat and is similar to that of a preterm infant at 28 weeks gestation (Patz, 1957). Retinal vessels of the newborn kitten are exquisitely sensitive to oxygen tension, and they severely vasoconstrict in response to extreme hyperoxia. Under continued hyperoxia, this responsiveness persists until the vessels become mature. The pattern of vasoproliferation in the kitten retina is distinctly different from that of the human. Like the mouse and rat models, retinal detachment rarely occurs in the kitten retina (Gole et al., 1982). In contrast to the kitten, the beagle puppy retina is more vascularized at birth, and the rate of vascularization is faster. However, the pattern and severity of reaction to extreme hyperoxia is similar to that of 
kittens (Flower et al., 1985; McCleod et al., 1998). Finally, retinal detachment occurs with greater frequencyin the beagle model than in other species.

Vascular endothelial growth factor expression patterns are disrupted in both the rat and mouse models of OIR. In situ hybridization and western blot experiments have shown VEGF expression to be decreased during the hyperoxic phase of oxygen-exposed mice compared to normal control animals, with an increase in VEGF mRNA and protein expression in the Müller cells of the inner nuclear layer within six hours of removal to room air (Pierce et al., 1995; Pierce et al., 1996; Ozaki et al., 1999). Notably, HIF-1 expression is increased in the inner nuclear layer prior to any increase in VEGF mRNA expression in OIR mice (Ozaki et al., 1999). VEGF levels remain high throughout the period of neovascularization in the mouse and rat models. VEGF mRNA as measured by in situ hybridization is increased in the inner nuclear layer, especially around the Müller cells, at a time preceding neovascular growth in the rat model (Dorey et al., 1996), as is protein detected by immunohistochemistry (Robbins et al., 1997). A similar increase in Flt-1 and Flk-1 protein occurs with the onset of neovascularization on day 16 (Robbins et al., 1998). In mice, intravitreal administration of exogenous VEGF prior to oxygen exposure resulted in a decrease in the vasoattenuation normally associated with hyperoxia, thus indicating that VEGF is required for vessel growth and survival in this scenario (Pierce et al., 1996). Similarly, a decrease in VEGF expression in the astrocytes and Müller cells was seen in P10 rats exposed to $80 \%$ oxygen. Administration of exogenous $\mathrm{VEGF}_{165}$ prior to exposure to hyperoxia rescued the vessels from hyperoxia-induced apoptosis and vasoattenuation, thus providing hope for the therapeutic use of VEGF or VEGF analogs in preventing regression of vessels in the early phase of ROP (Alon et al., 1995). Likewise, inhibition of VEGF by injection of VEGF receptor chimeric proteins, anti-VEGF antibodies, or antisense oligonucleotides in the mouse model of retinopathy during the neovascular phase has been shown to decrease abnormal blood vessel growth (Aiello et al., 1995b; Adamis et al., 1996; Robinson et al., 2001).

Finally, in an attempt to limit retinal hypoxia during the late, vasoproliferative phase, investigators have administered supplemental oxygen to animals (kitten, mouse, rat) during the period of recovery in room air. Generally, this treatment has been shown to attenuate neovascularization, and it appears to do so by decreasing VEGF expression (Phelps, 1988; Berkowitz and Zhang, 2000; Chan-Ling et al., 1995a; Pierce et al., 1996; Stone et al., 1996). These findings inspired the implementation of the STOP-ROP multicenter clinical trial described below.

\subsection{Other Factors and Retinal Vascular Development}

Other factors, such as IGF-1 (discussed above) play important roles in the development of blood vessels. Some of these factors may exert their influences by VEGF-dependent mechanisms. For example, erythropoietin, angiopoietin and Platelet-derived growth factor all regulate aspects of vascular development.

2.6.1 Erythropoietin (EPO)—EPO is a glycoprotein hormone produced in the fetal liver and adult kidney, which plays a major role in increasing production of red blood cells in response to anemia or hypoxia (Krantz, 1991). Expression of EPO and EPO receptor mRNA is present in the neural retina as early as 8 weeks gestation, although its role remains unclear (Juul et al., 1998). EPO gene expression is inducible by hypoxia. This increase is mediated by HIF-1a and HIF-1a like factor (HLF) (Wang and Semenza, 1995; Ema et al., 1997). Indirect evidence for the role of EPO in retinal neovascularization comes from studies of HLF knock down mice (HLF +/-), with 20\% HLF expression in the retina compared to levels in wild type mice. Under normoxia, the retinal vasculature of these mice is similar to 
that of wild type mice. However, in mice exposed to the oxygen-induced retinopathy paradigm, EPO mRNA (in contrast to VEGF mRNA) is reduced during the post-oxygen exposure phase and retinal vascularization is almost completely suppressed (Watanabe et al., 2005). Likewse, intraperitoneal injection of EPO into the HLF +/- mice increased the formation of neovascular tufts. In contrast, intraocular injections of soluble EPO receptor at day 17 (maximal neovascularization) in the oxygen-induced retinopathy wild type mice decreased neovascularization in a dose dependent manner. These studies indicate that VEGF alone is not adequate for neovascularization and that the interplay between VEGF and growth factors such as EPO is important for oxygen induced retinopathy.

Other evidence comes from clinical studies showing increased vitreous EPO levels in diabetic retinopathy compared to non-diabetic retina (Watanabe et al., 2005). Preterm infants are often treated with EPO to prevent anemia. The incidence of severe ROP was not increased in EPO-treated infants in several large clinical trials. However, a meta analysis of these trials showed that neovascular ROP is increased in EPO-treated infants (Ohlsson and Aher, 2006). A few single center studies have linked EPO use to increased incidence of ROP (Brown et al., 2006; Romagnoli et al., 2000). Collectively, these data suggest that EPO administration during the second phase of ROP may increase neovascularization.

2.6.2 Angiopoietin-The Angiopoietins (Ang-1 and Ang-2), along with the Tie2 receptor, constitute another ligand-receptor system in vascular development (Yancopoulos et al., 2000). Mice null for Ang-1 or Tie-2 die in utero (Sato et al., 1995; Suri et al., 1996). Although vascular tube formation occurs, the vessels remain immature from a lack of association of endothelial cells with underlying matrix and an inability to recruit supporting cells. Ang-2 is a competitive inhibitor of Ang-1. It binds to Tie-2 but is unable to initiate phosphorylation, thus providing a destabilizing signal for quiescent cells and allowing for remodeling of vessels either by regression in the absence of VEGF or angiogenesis in the presence of VEGF (Maisonpierre et al., 1997).

Ang-1 mRNA expression is high early in retinal vascular development and low in the later stages (P10-P16), while Ang 2 mRNA is low initially, peaks at P8 with development of the deep capillary plexus, and then plateaus. Ang- 2 is predominantly expressed by horizontal cells in the inner nuclear layer of the retina and a subset of amacrine and ganglion cells (Hackett SF 2000). In the oxygen-induced retinopathy mouse model, Ang-1 mRNA expression remains low during the post-oxygen exposure phase. In contrast Ang-2 mRNA, like VEGF mRNA, is increased within 6 hours of removal from hyperoxia and peaks at P17. Intravitreal injection of either sFlt-1 (to decrease VEGF) or sTie-receptor (to decrease Ang-2) or both, at P12 and P17, resulted in decreased neovascularization, but was maximal in animals injected with both sFlt-1 and sTie2-receptor (Takagi et al., 2003). Thus, inhibition of both VEGF and Ang-2 yields greater anti-angiogenesis.

2.6.3 PDGF-PDGF- $\alpha$ and PDGF- $\beta$ are mitogenic agents that recruit pericytes to the outer walls of blood vessels, increasing stability and allowing for branching (Betsholtz, 2004).

PDGF has the same pattern of expression as VEGF in the developing retina (Simpson et al., 1999). The role of PDGF in retinal angiogenesis is illustrated by PDGF null mice, which have reduced brain capillary pericytes and vessel abnormalities, including aneurysms (Lindahl et al., 1997).

In the rat ROP model, administration of a PDGF receptor inhibitor in the post-oxygen exposure phase resulted in increased VEGF and VEGFR-2 expression and increased retinal neovascularization (Wilkinson-Berka et al., 2004). Increased pericyte apoptosis and degeneration was also noted. Similarly, PDGF-deficient mice have an increased propensity for neovascularization (Hammes et al., 2002). Absence of pericytes possibly weakens the 
vessel wall producing tissue hypoxia followed by increased VEGF expression and neovascularization.

\subsection{Implications for Prevention and Treatment of ROP}

The above studies suggest that an exaggeration of normal compensatory mechanisms results in ROP. A decrease in VEGF expression in response to relative hyperoxia occurs in the first phase of ROP. This is followed by retinal hypoxia, leading to a compensatory increase in VEGF expression seen in the second phase of ROP, ultimately producing neovascularization. These findings have important implications for designing future preventive and treatment strategies for ROP. Treatments designed to prevent early phase ROP should avoid conditions that result in decreased VEGF or should consist of administration of VEGF analogs. Treatment of ROP during the vasoproliferative phase, should consist of strategies that decrease retinal hypoxia or employ the use of VEGF antagonists or other selective antiangiogenic agents that prevent abnormal, but allow for normal blood vessel growth. One feature that makes ROP amenable to treatment is the welldefined, short time period of insult and resultant neovascularization. One of the concerns of using VEGF or VEGF analogs during the first phase of ROP is the risk of stimulating abnormal blood vessel growth with increased permeability in other tissues and organs. However, the local delivery of VEGF analogs to the effected vessels, for example topically or by intravitreal injection, provides hope for such therapy, should a selective analog become available.

Placental growth factor (PIGF), a member of the VEGF family, plays an important role in retinal vascular development and hyaloid regression. It shows a temporal expression pattern in the inner neural retina that is distinct from VEGF (Feeney et al., 2003). Unlike VEGF, PIGF binds selectively to VEGFR-1 and thus its ability to activate tyrosine phosphorylation of VEGFR-2 is limited (VEGFR-1 may still elicit its role in angiogenesis by heterodimerization with VEGFR-2 and VEGFR-3) (Rahimi et al., 2000; Autiero et al., 2003; Dixelius et al., 2003). PlGF apparently does not lead to increased angiogenesis in response to hypoxia (Clauss et al., 1996; Cao et al., 1997; Shibuya et al., 1999; Simpson et al., 1999). In a study by Shih and co-workers, using the mouse model of OIR, intravitreal administration of PIGF prior to hyperoxic exposure protected the retinal vessels from hyperoxia-induced vasoattenuation without increasing neovascularization (Shih et al., 2003). Although the details of the signal transduction pathways underlying this protection are unknown, it is possible that the combination of decreased VEGF along with an increase in VEGFR-1 expression in the hyperoxic retina allowed for the rescue of blood vessels without increased abnormal proliferation.

Studies in preterm infants have demonstrated a relationship between exposure to high levels of oxygen and development of ROP (Patz et al., 1952; Kinsey et al., 1977; Lucey and Dangman, 1984; Bancalari et al., 1987). Although the precise oxygen saturations to avoid remain unknown, avoiding hyperoxia, and particularly avoiding swings between hyperoxia and hypoxia, in the first few weeks of life has been shown to decrease the severity of ROP (Flynn et al., 1987; Askie and Henderson-Smart, 2000; Tin et al., 2001; Chow et al., 2003; Anderson et al., 2004; York et al., 2004). The relationship between hyperoxia and subsequent neovascularization appears more complex than suggested previously. In mice, exposure to hyperoxia results in retinal vasoattenuation, secondary to decreased VEGF (Claxton and Fruttiger, 2003). Studies in the neonatal rat model of retinopathy developed by Penn have shown that variable oxygen exposure results in a greater degree of neovascularization in comparison to constant hyperoxia (Penn et al., 1993; Penn et al., 1994; Penn et al., 1995). This occurred despite the existence of large areas of retinal avascularity in constant hyperoxia-exposed rats. It is possible that variable oxygen results in greater mitogenic activity or production of VEGF, or causes sequestration of VEGF during 
hyperoxic episodes, leading to an accumulation over time in the variable environment (Werdich et al., 2004; McColm et al., 2004). These experiments argue for tighter control of oxygen saturation fluctuations in premature infants.

In the mouse OIR model, Gu and colleagues noted a transition in the sensitivity of the retinal vessels to hyperoxia between P11 and P15 (Gu et al., 2002). Surprisingly, prolonged exposure to hyperoxia beyond this period resulted in complete and normal revascularization of the central ischemic retina unlike mice recovered after P12 in room air. In contrast to studies conducted in kittens (Phelps and Rosenbaum, 1984; Phelps and Rosenbaum, 1987), revascularization in prolonged hyperoxia-exposed mice occurred earlier compared to those raised in room air (Chan-Ling et al., 1995). Also, these animals did not show any loss of astrocytes in the central avascular retina (Gu et al., 2002). Similar studies have suggested a role for glial cells in retinal vascular development and neovascularization (Chan-Ling et al., 1992; Jiang et al., 1994; Jiang et al., 1995; McLeod et al., 1998). The sharp transition in the response to hyperoxia between $\mathrm{P} 11$ and $\mathrm{P} 15$ seen in the study by Gu suggests, as did the studies by Penn utilizing variable oxygen, that our understanding of the underlying mechanisms of hyperoxia-induced retinopathy remains incomplete. Notably, a similar process is seen in the human retina; peak neovascularization is seen mainly between $35-37$ weeks post-conceptional age and is independent of post-natal age (Palmer et al., 1991).

The rationale for the supplemental therapeutic oxygen for prevention of retinopathy of prematurity (STOP-ROP) trial consisted of avoiding retinal hypoxia, and thus VEGF production during the vasoproliferative phase of ROP (STOP-ROP Multicenter Study Group, 2000). Preterm infants with pre-threshold ROP were randomized to receive supplemental oxygen to keep their oxygen saturations either $88-94 \%$ or $96-99 \%$. However, there were no statistically significant differences in the rate of progression to threshold ROP between the two groups. There may be several reasons for the lack of demonstrated success of the treatment. First, supplemental oxygen may have been administered too late in the course of the disease. As mentioned above, results from animal studies have shown that the effect of hyperoxia on the retinal vasculature is dependent on the developmental stage. After a critical period, the vessels are no longer responsive to hyperoxia (Gu et al., 2002).

Selection criteria for including infants may not have been optimal, or the oxygen saturations chosen may not have been high enough to prevent retinal hypoxia and decrease VEGF production. Another possibility is that other factors besides VEGF, factors that are less oxygen sensitive, are involved in the pathogenesis of the vasoproliferative phase of ROP.

The use of antiangiogenic agents, which are currently administered for adult age-related macular degeneration, remains a possibility for patients with ROP. Clearly, systemic administration of antiangiogenic therapies is not practical in the context of ROP, as angiogenesis is necessary for organogenesis throughout the infant's body. Likewise, it will be important to limit uptake of locally injected antiangiogenic material from the eye into the circulation. Even if delivery of angiostatic therapies can be restricted to the retina, the potential exists for undesirable consequences. As previously stated, VEGF and its receptors also play important roles in the development of the neural retina. Mice treated with VEGFR antagonists demonstrate dystrophic retinal neurogenesis. Moreover, background angiogenesis is a critical component of normal retinal vascular development in the premature infant. An angiogenic inhibitor that is selective for abnormal blood vessels would be optimal, but has not been developed. Finally, when considering the development of angiostatic theapies it is important to understand how other factors (e.g., erythropoietin, angiopoietin, platelet-derived growth factor, etc.) participate in the control of normal differentiation and growth of the retinal vasculature, and how anti-VEGF and other approaches might influence the actions of these factors. These issues underscore the 
challenge of antiangiogenic therapy in the developing retina and suggest that it should be undertaken only with appropriate caution.

\section{Diabetic Retinopathy}

\subsection{Clinical Features of Diabetic Retinopathy}

Diabetic retinopathy is the most frequent complication of diabetes and the leading cause of blindness in developed countries worldwide. Approximately $75 \%$ of all diabetic patients show clinical signs of retinopathy within 15 years after onset of diabetes and more than $10 \%$ develop visual impairment within this period (Klein et al., 1984; Klein and Klein, 1995; Sjolie et al., 1997). In the United States, diabetic retinopathy accounts for $8 \%$ of legal blindness, making diabetes the leading cause of blindness in working age adults. Diabetic retinopathy has a clinical progression pattern characteristic of ischemic retinopathy (for review, see Aiello et al., 1998; Frank, 2004; Klein and Klein, 1997). The initial alterations are manifested by biochemical signs of oxidative stress and cellular signs of subclinical inflammation. The earliest vascular changes include adhesion of leukocytes to the vessel wall (leukostasis), aggregation of platelets, altered blood flow, degeneration of pericytes and thickening of basement membranes. Blockage of the retinal capillaries may cause localized hypoxia, triggering increased tissue production of angiogenic factors, including VEGF. The release of VEGF and other angiogenic factors causes loosening of the vascular endothelial cell-cell junctions, leading to increased vascular permeability. Paradoxically, despite the increased production of VEGF, a potent survival factor for endothelial cells, microvascular endothelial cells degenerate, leading to capillary closure and formation of acellular, nonperfused capillaries.

With disease progression, vascular alterations, such as microaneurysms, dot/blot hemorrhages, cotton-wool spots, venous beading and vascular loops are evident upon ophthalmoscopic examination. The vascular leakage increases, and blood and fluid accumulate within the retinal tissue, forming exudative deposits. This condition is referred to as non-proliferative diabetic retinopathy (NPDR). Tissue swelling caused by accumulation of excess interstitial fluid can alter metabolic processes and ion fluxes within retinal neurons and glia. When the edema affects the macula it can result in neuronal distortion leading to decreased visual acuity (for review, see (Kent et al., 2000). The condition of diabetic macular edema (DME) is somewhat more common in patients with type 2 diabetes (7.5\%) than with type 1 diabetes (5.9\%) (Hirai et al., 2008).

In some patients, the NPDR progresses to proliferative diabetic retinopathy (PDF). PDR is characterized by growth of new blood vessels on the surface of the retina, analogous to those that form during late ROP. The new blood vessels are fragile and may break, leaking blood into the neural retina and vitreous, thus clouding the vitreous and compromising vision. With advanced PDR, fibro-vascular scar tissue grows from the retinal surface into the vitreous cavity. If untreated, this can cause retinal detachment resulting in blindness.

While microvascular changes clearly play an important role in diabetic retinopathy, a growing body of evidence suggests that neural cells also show significant functional alterations early in the course of diabetes (for review, see (Antonetti et al., 2006)). Clinical studies have demonstrated altered electroretinograms and impairment of color vision, and contrast sensitivity even before the clinical diagnosis of diabetic retinopathy (Ghirlanda et al., 1997; Han et al., 2004). Recent studies using visual field testing have shown field defects in patients with little of no vascular signs of retinopathy. Studies in rats have shown that retinal neurons undergo apoptosis within one month after the onset of experimental diabetes (Barber et al., 1998). The Ins2 $2^{\text {Akita }}$ diabetic mouse model has been found to have 
increased levels of the apoptosis marker caspase 3 activity early in the disease process (Barber et al., 2005).

Multicenter clinical trials have firmly demonstrated that intensive insulin therapy reduces the risks of diabetes complications, including diabetic retinopathy (DCCT, 1993; UKPDS, 1998). These studies have shown that intensive insulin therapy and tight control of blood glucose levels dramatically reduces the risk of onset and delays the progression of diabetic retinopathy for patients with either type 1 or type 2 diabetes. The insulin therapy normalized not only hyperglycemia, but also prevented dyslipidemia and normalized insulin content as well as other factors, such as inflammatory cascades. Thus it is possible that a variety of factors in addition to hyperglycemia contribute to diabetes complications.

The specific causes of retinal injury and disease progression in diabetic patients are not yet clear. However, studies of clinical specimens and various experimental models strongly support the concept that the elevation of blood and tissue glucose levels stimulates the production of angiogenic factors. While many growth factors and cytokines have been implicated in retinal disease, VEGF has been identified as a primary mediator of the vascular alterations in diabetic retinopathy (for review, see Duh and Aiello, 1999; Frank, 2004; Miller et al., 1997). High glucose, oxidative stress, hypoxia and inflammatory reactions, all of which occur in diabetes, have been shown to induce increased expression of VEGF. Moreover, tissue VEGFR2, which transduces VEGF's permeability-inducing and growth-promoting signals, is significantly increased in diabetes (Gilbert et al., 1998; Hammes et al., 1998).

\subsection{Vascular Inflammation and Diabetic Retinopathy}

As has been noted above, diabetic retinopathy in patients is characterized by a period of vascular injury and increased permeability which may be followed by active proliferation of new vessels (Aiello et al., 1998; Frank, 2004). Both of these processes involve increases in the expression levels of VEGF (Caldwell et al., 2005; Duh and Aiello, 1999). Although experimental diabetes in rodent models does not progress to proliferative diabetic retinopathy, many of the early vascular changes seen in patients are evident, such as increased permeability, microaneurysm formation, and leukostasis. The latter is caused by up-regulation of inflammatory mediators including the leukocyte adhesion molecule ICAM-1 (Joussen et al., 2004). Studies have shown that VEGF requires ICAM-1 to induce early vascular changes in experimental diabetes (Lu et al., 1999; Miyamoto et al., 1999). Mice deficient in the genes encoding for the leukocyte adhesion molecules CD18 and ICAM-1 demonstrate significantly fewer adherent leukocytes in the retinal vasculature as well as fewer damaged RECs and less vascular leakage after 11 or 15 months of diabetes (Joussen et al., 2004). These data strongly suggest that chronic, low-grade, sub-clinical inflammation is responsible for many of the vascular lesions seen in patients with diabetic retinopathy.

An association between the development of diabetes-related cardiovascular complications and increased expression of inflammatory markers has been well established by populationbased studies. Indeed sub-clinical chronic inflammation is considered to be a constant partner of the hyperglycemic milieu and is also found in other pathological conditions including obesity, hypertension and atherosclerosis (Alexandraki et al., 2006). Evidence of inflammation is also found in clinical specimens from patients with diabetic retinopathy (Esser et al., 1995; Lutty et al., 1997; Matsumoto et al., 2002; Meleth et al., 2005; Mitamura et al., 2001). In a recent study of 93 patients with diabetic retinopathy, severity of retinopathy was found to correlate with circulating levels of inflammatory cytokines as well as with plasma levels of triglycerides, cholesterol and hemoglobin $\mathrm{A}_{1 \mathrm{c}}$ (Meleth et al., 2005). These observations further support the hypothesis that inflammation contributes to the 
development of diabetic retinopathy. Studies in animals and tissue culture models have strongly implicated the development of retinal vascular inflammation with diabetes-induced increases in VEGF expression (Joussen et al., 2002).

\subsection{Oxidative Stress and Diabetic Retinopathy}

Studies showing that diabetes-induced retinal vascular damage can be prevented by treatments that reduce reactive oxygen species (ROS) formation support the role of oxidative stress in diabetic retinopathy (Du et al., 2003; Kowluru et al., 2000; Kowluru et al., 2001). Diabetes and high glucose conditions can increase the formation of ROS by multiple biochemical pathways, including glucose auto-oxidation, activation of the polyol pathway which can decrease levels of reduced glutathione and/or increase advanced glycation end products and activation of PKC which activates NAD(P)H oxidase (Fig. 10) (Brownlee, 2001; Brownlee, 2005; Inoguchi et al., 2003a; Inoguchi et al., 2003b; Jain, 2006). All of these events have been suggested to be initiated by superoxide overproduction by the mitochondrial electron-transport chain (Brownlee, 2005). However, while mitochondrial-derived ROS are important for the initiation of oxidative stress responses in models of hyperglycemia, studies in other models indicate that activity of NADPH oxidase is required in order to sustain sufficient levels of ROS formation for the transduction of specific cellular responses (Kimura et al., 2005; Lee et al., 2006b; Schafer et al., 2003). Data showing increased activity of $\mathrm{NAD}(\mathrm{P}) \mathrm{H}$ oxidase in retinas and vascular tissues of diabetic patients and animals and in high glucose-treated endothelial cells suggest that $\mathrm{NAD}(\mathrm{P}) \mathrm{H}$ oxidase is an important source of ROS in the diabetic milieu (Al-Shabrawey et al., In Pressa; Al-Shabrawey et al., In Press-b; Al-Shabrawey et al., 2006; Ellis et al., 1998; Griendling et al., 2000; Inoguchi et al., 2003b; Sonta et al., 2004). Studies using a mouse model for ischemic retinopathy have revealed that superoxide production by $\mathrm{NAD}(\mathrm{P}) \mathrm{H}$ oxidase has a primary role in VEGF expression and retinal neovascularization (Al-Shabrawey et al., 2005). Moreover, activity of NAD(P)H oxidase has also been implicated in high glucosemediated increases in VEGF expression in the diabetic retina. Recent studies indicate that increased expression of the NAD(P)H oxidase catalytic subunit NOX2 (previously known as gp91phox), is correlated with early signs of diabetic retinopathy, including increases in ROS and VEGF, and breakdown of the blood-retinal barrier (Al-Shabrawey et al., In Press-a). Furthermore, inhibiting NAD(P)H oxidase or deleting NOX2 has been found to block the actions of diabetes in causing leukostasis and breakdown of the blood-retinal barrier (AlShabrawey et al., In Press-b).

Increased levels of oxidative stress have been linked to increased production of VEGF upon high glucose treatment in vitro and in the diabetic retina (El-Remessy, 2003b; Ellis et al., 1998; Ellis et al., 2000; Kuroki et al., 1996; Obrosova et al., 2001). The mechanisms by which oxidative stress contributes to VEGF overexpression are not fully understood. However, inhibiting nitric oxide synthase (NOS) has been shown to prevent signs of diabetic retinopathy in rats (Du et al., 2002; El-Remessy, 2003b; Kowluru et al., 2000), suggesting that formation of reactive nitrogen species plays a role in the pathology. Recent studies also indicate a role for peroxynitrite in diabetic retinopathy. Peroxynitrite is formed by the rapid combination of NO with superoxide at near diffusion levels. It is a highly reactive species that can alter protein function due to tyrosine nitration, thiol oxidation or S-nitrosylation reactions. Studies of endothelial cells treated with high glucose in culture showed that high glucose-induced superoxide formation leads to an increase in intracellular calcium that activates endothelial NOS (NOS3) to form NO, but also causes NOS3 "uncoupling" to generate superoxide as well as NO (Fig. 10) (El-Remessy, 2003a; Graier et al., 1999; Graier et al., 1997; Graier et al., 1996) resulting in peroxynitrite formation. Research demonstrating increased levels of the peroxynitrite biomarker nitrotyrosine in retinas of diabetic patients and experimental animal models supports this concept and implies a role for peroxynitrite in 
the development of diabetic complications (Abu El-Asrar et al., 2004; Du et al., 2002; ElRemessy, 2003b; Kowluru and Odenbach, 2004; Obrosova et al., 2005). Studies in animal and tissue culture models have demonstrated that increased peroxynitrite formation is directly correlated with diabetes, high glucose-induced VEGF expression, and increased retinal vascular permeability (El-Remessy, 2003a; El-Remessy, 2003b; El-Remessy et al., 2006; El-Remessy et al., 2005). This cycle can be interrupted by treatments that reduce superoxide or peroxynitrite levels or inhibit NOS activity. Further support for the role of peroxynitrite in diabetes-induced increases in VEGF expression is provided by studies showing that peroxynitrite increases VEGF expression in retinal endothelial cells (Platt et al., 2005).

\subsection{VEGF and Diabetic Retinopathy}

Studies of clinical specimens have shown a strong correlation between increases in intraocular VEGF levels and the development of PDR (for review, see Duh and Aiello, 1999). VEGF also is thought to have a key role in the pathogenesis of diabetic macular edema (DME) due to its action in increasing vascular permeability. Studies in diabetic animal models have shown that retinal vascular alterations resembling background diabetic retinopathy are associated with increases in retinal VEGF levels and increased expression of VEGFR2 (Gilbert et al., 1998; Hammes et al., 1998). Furthermore, blocking VEGF function can prevent diabetes-induced permeability increases in both patients (Avery et al., 2006; Haritoglou et al., 2006; Jorge et al., 2006; Starita et al., 2007) and animal models (Ideno et al., 2007; Ishida et al., 2003; Qaum et al., 2001), implying a direct role for VEGF in this pathology.

Again, VEGF receptors have been identified in retinal neurons, glia, microglia and RPE (Forstreuter et al., 2002; Hollborn et al., 2006; Robinson et al., 2001), and VEGF has been shown to play a key role in retinal development and survival of retinal neurons following ischemia/reperfusion injury (Nishijima et al., 2007; Yang and Cepko, 1996). As has been explained above, diabetes/high glucose stimulates increases in VEGF expression, and agents that block VEGF activity can prevent diabetes-induced retinal damage. However, because of VEGF's function in supporting neuronal as well as endothelial cell integrity, the actions of these anti-VEGF drugs may have undesirable side effects. A better understanding the molecular events specifically linked to pathological increases in VEGF expression is of fundamental importance for the development of new pharmacological tools to "fine tune" VEGF expression in the diabetic retina.

\subsection{Regulation of VEGF Gene Expression in Diabetic Retinopathy}

3.5.1 VEGF expression-VEGF gene expression is a tightly regulated process. Overexpression has profound pathological consequences in a variety of disease conditions, including diabetic retinopathy (Duh and Aiello, 1999; Miller et al., 1997). Thus, the biological activity of VEGF is tightly linked to its expression level. In the diabetic retina many factors can contribute to over-expression of VEGF, including tissue hypoxia, growth factors, inflammatory cytokines and reactive oxygen species. Each of these factors has been shown to modulate the activity of important molecular regulators of VEGF gene expression in other disease models. However, to date, we do not have a clear picture of how VEGF gene regulation is altered by diabetes. The following sections discuss the regulation of VEGF gene expression and its relevance in diabetes.

3.5.2 Transcriptional Regulation of VEGF Expression-Tissue hypoxia is certainly the most studied regulator of VEGF gene transcription. Studies in mice diabetic for five months have shown that diabetes-induced decreases in retinal capillary density are associated with retinal hypoxia as shown by localization and quantification of the hypoxia 
marker pimonidazole (de Gooyer et al., 2006) However, the role of hypoxia in the initiation of VEGF over-expression in the diabetic retina is uncertain in that increases in VEGF production are evident as early as one week after the induction of diabetes, which is long before any signs of retinal capillary closure or REC dropout are evident. The early onset of VEGF upregulation may imply a role for inflammatory and redox stimuli in promoting VEGF expression. On the other hand, it has been suggested that hyperglycemia-induced leukostasis could result in multiple foci of transient hypoxia due to reversible occlusion of the retinal capillaries by adherent leukocytes (Joussen et al., 2004).

As previously stated, transcriptional regulation of the VEGF gene in hypoxic conditions has been mainly attributed to activity of the heterodimeric helix-loop-helix transcription factor hypoxia-inducible factor-1 (HIF-1) (Forsythe et al., 1996). Little is known about the role of HIF-1a in diabetes-induced upregulation of VEGF expression in the retina. However, HIF-1a has been implicated in disruption of the blood-retinal barrier in diabetic animals treated with intensive insulin therapy during early diabetes (Poulaki et al., 2002).

In addition to hypoxia, a number of factors can contribute to the over-expression of VEGF during diabetic retinopathy, including TGF- $\beta$, TNF- $\alpha$, IGF-1, advanced glycation end products and oxidative stress, all of which are increased during diabetes (Fig. 11) (Cohen et al., 1996; Goad et al., 1996; Hoffmann et al., 2002; Li et al., 1995; Pertovaara et al., 1994; Ryuto et al., 1996). The mechanisms by which these factors contribute to up-regulation of retinal VEGF transcription are not yet clear. However, ROS have been shown to induce VEGF transcription by a process involving the activity of STAT3 (signal transducer and activator of transcription factor 3 (Fig. 12) (Al-Shabrawey et al., submitted; Bartoli et al., submitted; El-Remessy, 2003b; El-Remessy et al., 2006; Platt et al., 2005). STAT3 has been implicated in the stimulation of angiogenesis by inducing VEGF expression (for review, see Chen and Han, 2007). In addition, VEGF induces activation of STAT3 and promotes its autocrine expression in RECs (Bartoli et al., 2000; Bartoli et al., 2003). Of interest, STAT3 activation is stimulated by inflammatory pathways (Hirano et al., 2000). Importantly, STAT3 has been shown to play a critical role in the process of HIF-1a activation and VEGF expression in multiple growth signaling pathways (Xu et al., 2005). In conditions of diabetes or high glucose treatment, STAT3 activation occurs in a redox-dependent manner involving $\mathrm{NAD}(\mathrm{P}) \mathrm{H}$ oxidase activity and peroxynitrite formation (Al-Shabrawey et al., submitted; Bartoli et al., submitted; Platt et al., 2005). These observations imply that redox-dependent activation of STAT3 may play a very significant role in the induction of VEGF expression in the diabetic retina in response to both inflammatory and hypoxic conditions.

In addition to HIF-1 and STAT3, several other transcription factors and co-activators have been implicated in the regulation of VEGF expression, including Sp1 (Shi et al., 2001) and AP-1 (Damert et al., 1997). Both can work alone or in coordination with other transcriptional regulators. Hypoxia, oxidative stress and cytokines can all increase the activity of these transcriptional regulators, suggesting their potential contribution to the induction of VEGF expression in the diabetic retina.

3.5.3 Post-transcriptional Regulation of VEGF Expression-The importance of post-transcriptional events in the regulation of the VEGF gene in normal and pathological conditions is well established. However, very little is known about whether and how diabetes and high glucose may alter VEGF post-transcriptional regulation. Whether posttranscriptional mechanisms are involved in hyperglycemia-induced up-regulation of VEGF production remains to be determined. However, it is possible that both mechanisms mRNA stabilization and activation of multiple internal ribosomal entry sites (IRES) - are involved. The role of the RNA-binding protein HuR in the up-regulation of VEGF production in the diabetic retina is supported by evidence linking this molecular mechanism 
to VEGF production in response to stress and hypoxia. IRES-dependent production of alternatively spliced VEGF isoforms may also contribute to the pathological effects of VEGF in the diabetic retina.

3.5.4 VEGF Alternative Splicing-It has been suggested that the different VEGF splice variants play different roles in retinopathy in that $\mathrm{VEGF}_{165}$ is a potent inducer of retinal vascular inflammation as well as a mediator of neuronal survival, whereas VEGF $_{121}$ promotes neuronal survival, but does not induce vascular inflammation (Ishida et al., 2003; Nishijima et al., 2007). Decreased levels of the dominant negative splice variant VEGF $_{165 b}$ were found in vitreous samples from patients with diabetic retinopathy, suggesting that a molecular switch between the $\mathrm{VEGF}_{165}$ and $\mathrm{VEGF}_{165 \mathrm{~b}}$ could be involved the development of diabetic retinopathy (Perrin et al., 2005). More study is needed to define the specific molecular events that regulate VEGF mRNA splicing and to see how this process is altered in the diabetic retina.

3.5.5 VEGF autocrine expression in endothelial cells-VEGF expression has been described mainly as a paracrine event. However, VEGF's autocrine ability to stimulate its own production in the microvascular endothelium has been described in hypoxia, brain tumors, when the cell-to-cell junctions are disrupted or during in vitro angiogenesis induced by advanced glycation end products (AGE) (Schaefer et al., 2002; Yamagishi et al., 1997). Moreover, studies in mouse support a role for neuronal VEGF in both paracrine and autocrine signaling in the maintenance of neurons and endothelial cells in the developing central nervous system (Ogunshola et al., 2002) Studies of retinal glial cells have shown that hypoxia-induced expression of MT1-MMP is mediated by VEGF in an autocrine fashion (Noda et al., 2005). The role of VEGF autocrine expression in diabetic retinopathy is not yet known, but high glucose and peroxynitrite both appear to induce VEGF expression in cultured RECs (Bartoli et al., submitted; Platt et al., 2005). It appears likely that pathological overgrowth of the retinal microvasculature caused by diabetes is due in part to VEGF's autocrine actions triggering VEGF overexpression. Parallel actions of high glucose and oxidative stress might inhibit the production of proteins that normally inhibit VEGF expression, enhancing this autocrine process. This hypothesis is supported by studies showing that decreased levels of endostatin and PEDF are correlated with increased VEGF levels in patients with diabetic retinopathy (Boehm et al., 2003; Funatsu et al., 2003) and that treatment with endostatin or PEDF can reduce VEGF- or AGE-induced retinal vascular permeability in vivo (Takahashi et al., 2003; Yamagishi et al., 2006).

\subsection{Regulation of VEGF Function in Diabetic Retinopathy}

3.6.1 VEGF and Vascular Inflammation-VEGF is a potent inducer of vascular inflammation. VEGF treatment of endothelial cells in vitro increases their expression of ICAM-1 and MCP-1 (Hong et al., 2005; Lu et al., 1999; Melder et al., 1996). The role of VEGF in causing inflammation during diabetic retinopathy has also been demonstrated by studies showing that increases in VEGF expression are correlated with increased levels of ICAM-1 immunoreactivity and leukostasis in the retinas of diabetic animals (Hammes et al., 1998; Joussen et al., 2001; Joussen et al., 2002; Miyamoto et al., 1999; Murata et al., 1996; Qaum et al., 2001). The VEGF $_{165}$ isoform is thought to be critically involved in the retinal vascular inflammatory response to diabetes in that isoform-specific blockade of $\mathrm{VEGF}_{165}$ suppresses leukostasis and restores normal blood-retinal barrier function in diabetic rats (Ishida et al., 2003). This concept is further supported by data showing that intravitreal injection of $\mathrm{VEGF}_{165}$ induces expression of ICAM-1 in the retinal vasculature of mice (Lu et al., 1999) and increases ICAM-1 levels and leukostasis in rats (Ishida et al., 2003). 
3.6.2 VEGF and Vascular Permeability-During diabetes, breakdown of the bloodretinal barrier occurs at the level of the vascular endothelium (for review, see Antonetti et al., 1999b). The permeability defect has been correlated with increased ocular levels of VEGF in clinical specimens and experimental animals (Adamis et al., 1994; Aiello et al., 1994; El-Remessy, 2003b; Qaum et al., 2001; Takeda et al., 2001). Studies with a variety of VEGF blocking strategies have demonstrated the role of VEGF in the permeability response of diabetic animals (Ideno et al., 2007; Ishida et al., 2003; Qaum et al., 2001). Clinical trials in patients with diabetic macular edema have shown beneficial effects of anti-VEGF agents in reducing retinal vascular permeability as assessed by fluorescein angiography and decreasing macular edema as shown by optical coherence tomography (Avery et al., 2006; Haritoglou et al., 2006; Jorge et al., 2006; Starita et al., 2007). Recent data indicate that intravitreal injections of PEDF can also reduce vascular hyper-permeability in diabetic rats by a process associated with decreased levels of a variety of inflammatory mediators, including VEGF, VEGFR-2, MCP-1, TNF-a and ICAM-1 (Zhang et al., 2006a, 2006b).

Studies of the mechanisms by which VEGF induces the vascular permeability increase indicate that multiple pathways are involved. In an in vivo study in which VEGF was applied topically or by intradermal injection, Roberts and Palade showed that VEGF treatment is followed by a rapid increase in permeability of capillaries and post-capillary venules that is associated with the formation of fenestrae, a trans-cellular permeability route (Roberts and Palade, 1995). Subsequent in vitro work with cultured RECs showed that VEGF treatment caused a rapid, but transient, increase in transcellular permeability that resolved within 1 hour, followed by a delayed but sustained increase in paracellular permeability beginning 4-6 hours after VEGF treatment (Fig. 13) (Behzadian et al., 2003; Feng et al., 1999a). The transient increase in transcellular permeability was found to be mediated by a mechanism of transcytotic transport in caveolin-coated vesicles that required activity of NOS3 and was accompanied by nuclear translocation of NOS3 and VEGFR2 (Fig. 14) (Feng et al., 1999a; Feng et al., 1999b). By contrast, the delayed increase in permeability was found to be mediated by a mechanism involving nuclear translocation of $\beta$-catenin (Fig. 15), increased expression of the receptor for urokinase plasminogen activator (UPAR) and pericellular proteolysis (Behzadian et al., 2003).

Earlier studies had indicated that TGF- $\beta$, which is known to increase VEGF expression (Behzadian et al., 1998), causes an increase in REC permeability by inducing the activation of MMP-9 (Behzadian et al., 2001). Membrane-bound pro-MMP-9 is activated by plasmin (Makowski and Ramsby, 1998) and VEGF induces expression of uPAR (Mandriota et al., 1995), which initiates plasmin formation. Therefore, it appears likely that the VEGFinduced permeability results from disruption of endothelial cell-to-cell attachments by uPAR-mediated initiation of a cascade of proteolytic activities on the cell surface (Fig. 16). This pericellular proteolysis alters cell-cell and cell-substrate attachments, generating leaky vessels and allowing the endothelial cells to penetrate the underlying basement membrane, migrate and proliferate, thereby setting the stage for retinal neovascularization.

The above in vitro results are consistent with in vivo data showing that diabetes-induced increases in vascular permeability are associated with increases in expression of VEGF and uPAR and with oxidative stress as evidenced by increased formation of NO, superoxide and peroxynitrite (El-Remessy, 2003b). This work also showed that inhibiting NOS activity or scavenging peroxynitrite blocked the diabetes-induced increase in oxidative stress, normalized VEGF and uPAR expression and preserved the blood-retinal barrier. These results imply a causal relationship between oxidative stress, VEGF overexpression, uPA/ uPAR activation and hyperpermeability. The potential role of the uPA/uPAR system in diabetic retinopathy has also been supported by studies showing that the diabetes-induced increase in retinal vascular permeability does not occur in diabetic mice that lack the uPAR 
gene (Behzadian et al., 2004) or in diabetic rats treated with a uPAR blocker (Navaratna et al., 2007).

Identifying the downstream mediators of the VEGF-induced permeability increase is important for the development of new treatment strategies. The role of an alteration in endothelial cell junction adhesion in diabetes-induced permeability has been supported by studies showing a decreased level of the tight junction protein occludin in rats rendered diabetic with streptozotocin or treated with intraocular injections of VEGF (Antonetti et al., 1998; Barber et al., 2000). The VEGF-induced hyperpermeability response appears to be associated with phosphorylation of occludin and another junction protein, ZO-1 (Antonetti et al., 1999a). Subsequent work has demonstrated that VEGF-induced phosphorylation of occludin involves the activation of PKC and that phosphorylation of occludin is also associated with hyperpermeability in diabetes (Harhaj et al., 2006). Studies in tissue culture cells have further suggested that VEGF-induced increases in endothelial cell permeability involves phosphorylation of VE-cadherin which results in beta-arrestin-dependent endocytosis of VE-cadherin (Gavard and Gutkind, 2006). A similar mechanism may be involved in diabetes-induced permeability increases in that treatment of endothelial cells with advanced glycosylation end products has been shown to cause a reduction of VEcadherin staining on the cell surface and increased permeability (Navaratna et al., 2007a).

Studies of the VEGF signaling pathway in vascular endothelial cells indicate that activation of VEGFR-2 involves the formation of protein complex between VEGFR-2 and c-Src, and c-Src activation is required for VEGF induction of NO and prostacyclin formation (He et al., 1999). Further studies demonstrated that VEGF stimulation of cultured RECs promotes expression of the UPAR gene by inducing and transcriptional activation of $\beta$-catenin and showed that the VEGF-induced increase in paracellular permeability involves activation of the uPA/uPAR system (Behzadian et al., 2003). The role of $\beta$-catenin-mediated uPAR expression in VEGF-induced hyperpermeability is supported by data showing that the action of PEDF in blocking VEGF-induced permeability increases involves the inhibition of $\beta$ catenin cytosolic redistribution and nuclear translocation and blockade of uPAR expression (Yang et al., 2007). Interestingly, recent data suggest that inhibition of VEGF-induced permeability by PEDF involves upregulation of gamma-secretase activity and a decrease in VEGF-induced association of VEGFR-1 with $\beta$-catenin and VE-cadherin (Boulton et al., 2007). Further definition of the VEGF-induced signaling pathways leading to these events should aid in the development of more specific and efficient therapies for the treatment of diabetic macular edema.

3.6.3 VEGF and Retinal Angiogenesis-The action of VEGF in stimulating angiogenesis is well established (Ferrara, 1999; Ferrara, 2001). However, recent data indicate that alterations in vasculogenesis could also be involved in diabetic retinopathy (Asahara et al., 1997; Butler et al., 2005; Guthrie et al., 2005; Kelly et al., 2005; Lee et al., 2006a). In this context, vasculogenesis refers to the process by which circulating bone marrow-derived endothelial progenitor cells (EPCs) are recruited into growing vessels. In vitro studies of EPCs isolated from diabetic patients have shown impairment in their proliferation, adhesion and incorporation into vascular structures, suggesting that EPC alterations may contribute to vascular dysfunction during diabetes (Tepper et al., 2002). Studies in a mouse model of diabetes showed impairment of vasculogenesis in response to hind limb ischemia (Schatteman et al., 2000). Studies in a model of ischemic retinopathy have shown that bone marrow-derived hematopoietic stem cells participate in retinal neovascularization (Grant et al., 2002). Investigations using cell-based gene therapy in the mouse OIR model showed that these cells can rescue and stabilize degenerating vessels, and the cells can inhibit new vessel formation in a mouse model of retinal degeneration (Otani et al., 2002). By contrast, studies using animal models for ocular vascular damage, including 
diabetes, ischemia/reperfusion injury and OIR, have shown that cells from normal donors attach and assimilate into the existing vasculature. However, cells from diabetic donors uniformly were unable to integrate into damaged vasculature (Caballero et al., 2007). These findings further indicate that healthy EPCs can effectively repair injured retina and that there is defective repair of vasculature in patients with diabetes.

3.6.4 VEGF and Cell Survival-The function of VEGF in promoting endothelial cell survival has been well documented (Duh and Aiello, 1999). However, even though levels of VEGF and VEGFR2 are increased in the diabetic retina, endothelial cell survival remains compromised, as shown by formation of acellular capillaries and increased numbers of apoptotic RECs (Duh and Aiello, 1999; Mizutani et al., 1996). This apparent contradiction between increased VEGF expression and impaired endothelial cell survival suggests that the VEGF pro-survival signaling pathway in RECs may be altered by the diabetic milieu.

The VEGF pro-survival pathway is mediated by VEGFR-2-dependent activation of the PI3K/Akt signaling pathway (Gerber et al., 1998a; Gerber et al., 1998b). However, VEGFinduced activation of VEGFR2 also activates the serine/threonine protein kinase p38 MAPK (Rousseau et al., 1997). This kinase is an important modulator of the pro-apoptotic process in various cell types, including endothelial cells. Blockade of VEGF-induced PI3K or Akt signaling enhances the activation of p38 MAPK, leading to increased apoptosis (Gratton et al., 2001). Treatment of RECs with high glucose medium or exogenous peroxynitrite has been shown to trigger a similar phenomenon of accelerated apoptosis even in the presence of exogenous VEGF (El-Remessy et al., 2005; Gu et al., 2003). This pro-apoptotic effect is correlated with increased phosphorylation of p38 MAPK, decreased Akt-kinase activity, and tyrosine nitration of the P85 and P110 subunits of PI3K, implying that peroxynitrite in high glucose impairs endothelial cell survival (Fig. 17) (El-Remessy et al., 2005). In vitro studies have shown that the $\mathrm{p} 85$ regulatory subunit of PI3K is a target for peroxynitrite-induced tyrosine nitration and that tyrosine nitration blocks the interaction of $\mathrm{p} 85$ with the $\mathrm{p} 110$ catalytic subunit (Hellberg et al., 1998), indicating that peroxynitrite can alter cell survival responses mediated by PI3K. Increased levels of tyrosine nitration are also observed in retinal proteins of diabetic rats (El-Remessy, 2003a; El-Remessy, 2003b), suggesting that peroxynitrite plays an important role in mediating the suppressive effects of diabetes on endothelial cell survival. These results suggest that peroxynitrite may be ultimately responsible for the pathophysiology previously attributed to NO alone (Misko et al., 1998; Salvemini et al., 1998).

\subsection{Targeting VEGF in Diabetic Retinopathy}

3.7.1 Laser therapy-Laser photocoagulation is the recommended treatment for clinically significant DME or advanced PDR (for review, see Frank, 2002; Frank, 2004). This treatment is presumed to work by destroying retinal tissue and thereby decreasing metabolic demand and diminishing retinal hypoxia and VEGF expression. While the therapy is effective in most patients, the loss of retinal tissue can decrease peripheral vision, impair night vision and change color perception. Moreover, the retinopathy will sometimes progress despite appropriate treatment. Therefore, there is a great need for the development of new therapies to prevent and treat diabetic retinopathy.

3.7.2 Inhibiting PKC—Preclinical studies have provided strong evidence implicating the activation of PKC $\beta$ in the upregulation of VEGF expression during diabetic retinopathy (for review, see Sheetz and King, 2002). Large scale clinical trials testing the efficacy of the specific PKC $\beta$ inhibitor LY333531 for patients with diabetic retinopathy showed that the treatment reduced vision loss, need for laser treatment and macular edema progression, 
while increasing occurrence of visual improvement in patients with nonproliferative retinopathy (Aiello et al., 2006).

3.7.3 Inhibiting VEGF Function-Macugen and Lucentis have been approved by the FDA for treatment of AMD (see section 4.11). Avastin, while approved only for systemic treatment in cancer, has also been found to be effective for AMD. Although these agents have not been specifically approved for use in diabetic patients, clinical trials are in progress to test their efficacy in treatment of DME. In a phase II clinical trial, patients treated with intraocular injections of Macugen had better visual acuity outcomes, were more likely to show reduction in central retinal thickness and were deemed less likely to need additional therapy with photocoagulation at follow-up as compared with the sham-injected control subjects (Cunningham et al., 2005). A study with ten patients showed that intraocular injections of Lucentis significantly reduced foveal thickness and improved visual acuity (Nguyen et al., 2006). In another study of 32 patients, intravitreal injections of Avastin were associated with a rapid regression of retinal and iris neovascularization secondary to PDR (Avery et al., 2006). Another small uncontrolled study showed improvement of visual acuity and decreased retinal thickness after intravitreal injection of Avastin (Haritoglou et al., 2006). Yet another study showed that intravitreal Avastin injections were followed by reduction of fluorescein leakage from persistent active neovascularization without loss of vision in patients with diabetic retinopathy (Jorge et al., 2006). While strategies to increase PEDF levels have shown promise for the treatment of wet AMD (Campochiaro, 2007), this approach has not been tested in diabetic patients.

3.7.4 Targeting VEGF expression-An important goal for future drug development work will be to develop pharmacological tools to fine tune VEGF expression in diseased tissue, including the diabetic retina. This might be addressed by inhibiting transcription or translation of specific VEGF isoforms, by increasing and/or preserving levels of $\mathrm{VEGF}_{165 \mathrm{~b}}$, by blocking HuR binding to the nascent VEGF mRNA or by suppressing IRES-dependent translation.

While drugs that specifically target components of the VEGF transcriptional and translational machinery are not yet available for use in diabetic patients, this strategy has shown promise for the treatment of cancer. For example, several inhibitors of HIF-1 activity have been developed and tested for their efficacy in blocking tumor neovascularization (Rapisarda et al., 2002). The immunosuppressant rapamycin has been shown to exhibit antiangiogenic activity by suppressing VEGF expression (Guba et al., 2002). The effects of rapamycin on VEGF expression involve the inhibition of PI3K/Akt-dependent mTOR (mammalian target of rapamycin) activation, which results in decreased transactivating function of HIF-1 (Hudson et al., 2002; Treins et al., 2002; Zhong et al., 2000).

Recent data indicate that inhibitors of 3-hydroxy-3-methylglutaryl-coenzyme A (HMG$\mathrm{CoA}$ ) reductase, lipid lowering drugs known as statins, can prevent VEGF over-expression in the diabetic retina (Al-Shabrawey et al., submitted; Miyahara et al., 2004). The beneficial effects of statin treatment in the diabetic retina were found to correlate with their ability to suppress redox-dependent activation of STAT3 (Al-Shabrawey et al., submitted). Statins have also been shown to inhibit angiotensin-induced activation of STAT3 in vitro (Horiuchi et al., 2003) and to prevent VEGF expression induced by advanced glycation end-products (Okamoto et al., 2002; Scalia and Stalker, 2002; Weis et al., 2002). These and other important therapeutic effects of statins are due not only to their lipid lowering ability, but also to their potent antioxidant and anti-inflammatory properties (Sowers, 2003). These pleiotropic effects involve the inhibition of the isoprenylation/activation of small $\mathrm{G}$ proteins RhoA and Rac-1 (Liao, 2002). Statin-mediated inhibition of RhoA and Rac-1 restores normal redox conditions in vascular endothelial cells by normalizing NOS3 expression and 
activity and by blockade of Rac-1-induced NAD(P)H oxidase activity (Delbosc et al., 2002). Together, these observations suggest that STAT3 activation and STAT3-dependent VEGF expression in the diabetic retina are downstream to the elevation of ROS by the superoxide generator NAD $(\mathrm{P}) \mathrm{H}$ oxidase (Al-Shabrawey et al., submitted). Therefore, the protective effects of statin therapy against diabetic retinopathy can be attributed to its antioxidant activity and ability to inhibit STAT3 activation. The efficacy, side effects and toxicity of statins are well established after more than a decade of clinical use. Specific clinical studies determining their efficacy in preventing diabetic retinopathy will be important.

Blockade of STAT3 function prevents diabetes-induced increases in VEGF expression, arrests leukostasis and preserves normal blood-retinal barrier function in rats (Bartoli et al., submitted). Therefore, direct blockade of STAT3 activation in the retina should be considered as a potential treatment for diabetic retinopathy. Drugs targeting the activation of STAT3 directly have not been tested in diabetic retinopathy, but are under development for the treatment of cancer (for review, see Deng et al., 2007; Klampfer, 2006).

3.7.5 Antioxidants-As has been discussed above, the role of oxidative stress in diabetic vascular dysfunction and VEGF-over-expression is well established. This concept is supported by data showing that antioxidants such as vitamin E prevent some of the vascular dysfunction associated with diabetes in animal models (Kunisaki et al., 1995). In one study of diabetic patients, treatment with high-dose vitamin $\mathrm{E}$ for four months reversed diabetesinduced alterations in retinal blood flow (Bursell et al., 1999). However, studies on the effect of antioxidant supplementation in diabetic patients have largely been limited to examination of blood levels of biochemical markers of oxidative stress and other risk factors for retinopathy (Jain, 2006). Therefore, the implications of the data for the treatment of diabetic patients remain uncertain. Clearly, a more precise definition of the specific oxidants induced by diabetes and their molecular targets is needed in order to develop more effective treatments.

Recent studies in diabetic animals have focused attention on peroxynitrite as a therapeutic target for reducing oxidative stress during diabetic retinopathy. Studies have shown that diabetes- and high glucose-induced dysfunction in the peripheral vasculature involves the inactivation of endothelium-derived NO by its combination with superoxide anion to form peroxynitrite. This concept has been supported by research showing nitrotyrosine formation in the plasma of diabetic patients (Ceriello et al., 2001). Elevated nitrotyrosine levels have also been observed in blood vessels and retinas from diabetic rats (El-Remessy, 2003b; ElRemessy et al., 2006; Kowluru, 2003; Kowluru and Koppolu, 2002; Soriano et al., 2001). Moreover, exogenous peroxynitrite increases VEGF expression in cultured RECs (Platt et al., 2005). Increases in retinal nitrotyrosine levels and lipid peroxidation have been shown to correlate with VEGF overexpression and breakdown of the blood-retinal barrier, and treatments that reduce peroxynitrite formation have been shown to block the early signs of diabetic retinopathy (El-Remessy, 2003b; El-Remessy et al., 2006).

Moreover, VEGF itself has been shown to promote the formation of superoxide anion and peroxynitrite in cultured endothelial cells (El-Remessy et al., 2007; Ushio-Fukai et al., 2002). Furthermore, formation of both oxidants appears to be required for transduction of VEGF's angiogenic signal. Thus, treatments that block the formation of superoxide and peroxynitrite may improve diabetic retinopathy by inhibiting the VEGF intracellular signaling pathway. 


\section{Age-related Macular Degeneration (AMD)}

\subsection{AMD Overview}

Age-related macular degeneration (AMD) is one of the leading causes of vision loss worldwide (Resnikoff et al., 2004). The neovascular form comprises only $10 \%$ of AMD, but accounts for close to $90 \%$ of legal blindness from it. Recently, chemotherapeutic agents that inhibit actions of VEGF have been found to be effective in improving vision in approximately $40 \%$ of eyes with neovascular AMD, whereas previous modalities (i.e., photocoagulation, photodynamic therapy, radiation therapy, angiostatic steroids, corticosteroids and other anti-angiogenic drugs) were considered successful when vision did not decline and resulted in marginal improvements in vision less than $15 \%$ of the time (Blinder et al., 2003; Marcus and Peskin, 2004; D'Amico et al., 2003; Gragoudas et al., 2004).

AMD develops late in life, yet is strongly associated with certain common genetic mutations (Klein et al., 2005; Maller et al., 2006; Li et al., 2006b; Haines et al., 2005; Edwards et al., 2005; Rivera et al., 2005). Evidence supports that both genetic and environmental factors play a role in the pathogenesis (Seddon et al., 2006b). The paradox of a strong genetic component in a disease with late onset suggests that cumulative insults throughout, or stressors late in, life are important in tipping the balance between expected clinical aging and abnormal macular changes in susceptible patients. It also offers the possibility of preventive therapy once the important stressors and knowledge of an individual's risk profile and susceptibilities are determined. From studies focused on either genetic or environmental influences in AMD, what appears common to neovascular AMD in both groups is the pathologic expression of VEGF.

4.1.1 VEGF and AMD—Although current treatments that target VEGF have demonstrated the best clinical outcomes to date (Dugel, 2006; Rosenfeld et al., 2006b; Rosenfeld et al., 2005), there is concern about broadly inhibiting VEGF activity in AMD. VEGF is a survival factor for the developing (Saint-Geniez et al., 2006) and mature retina (Maharaj et al., 2006). In addition, vision loss can occur from atrophic AMD and geographic atrophy, and patients with neovascular AMD can have geographic atrophy in the same eye (Sunness et al., 1999). Thus, inhibiting VEGF (a survival factor for endothelial and neural cells (Blaauwgeers et al., 1999; Witmer et al., 2003; Saint-Geniez et al., 2006; Korte et al., 1984; Sakamoto et al., 1995; Hoffmann et al., 2000)) could conceivably lead to geographic atrophy and poor visual acuity in some patients with neovascular AMD. It is, therefore, important to titer the degree of VEGF blockade or specifically and selectively inhibit one or a few of the angiogenic actions of VEGF when considering VEGF inhibition as a treatment strategy.

Current evidence supports the notion that VEGF plays a role in the pathogenesis of clinical neovascular forms of AMD. Included in this evidence are the following: 1) VEGF is increased secondary to certain stimuli that are linked epidemiologically to AMD or choroidal neovascularization (CNV) and genetic models of CNV also have increased expression of VEGF, 2) the role of pigment epithelium derived factor (PEDF) in neovascular AMD, such as through regulation of VEGF expression and receptor binding, and 3) the success of anti-VEGF therapies.

\subsection{VEGF in Outer Retinal Health}

VEGF is important in vascular development (Stone et al., 1995), survival of nascent retinal vessels (Saint-Geniez et al., 2006) and in maintaining stable mature vessels (Maharaj et al., 2006). In addition, evidence establishes VEGF signaling as important in the health of the $\mathrm{RPE}$, Bruch's membrane, and choroidal endothelial cells (CECs). In vitro studies 
demonstrate preferential release of VEGF from the basal aspect of the RPE (Blaauwgeers et al., 1999) and histopathologic sections show immunoreactivity of VEGFR-1, -2 , and -3 in the choriocapillaris endothelium (Witmer et al., 2003), evidence that VEGF and its receptors are present together under normal conditions. Further support comes from immunohistochemical localization of the phosphorylated or activated form of VEGFR-2 in the RPE/choroid during ocular development in the mouse (Saint-Geniez et al., 2006). These studies provide additional mechanistic support for historical studies demonstrating the interdependence of the choroid and RPE (Fig. 18) and the role of VEGF as a survival factor. Relevant results include: 1) that RPE induced tube formation of CEC (Sakamoto et al., 1995); 2) that conditional inactivation of VEGF in RPE prevented choriocapillaris development (Marneros et al., 2005); 3) that loss of the choriocapillaris occurred after selective toxins to the RPE (Korte et al., 1984); and 4) that a chimeric toxin to VEGF 165 caused both RPE and CEC death (Hoffmann et al., 2000). In addition, the finding that VEGF receptors are expressed by retinal neurons suggests that it is also important to inner retinal neuronal health (Witmer et al., 2003; Kim et al., 1999). Mice with a deletion in the hypoxia response element and rendered unable to express VEGF in response to hypoxia develop a motor neuron disease similar to human amyotrophic lateral sclerosis (Oosthuyse et al., 2001). This further demonstrates the importance of VEGF as a survival factor, not only for endothelial cells and RPE, but also for neurons.

\subsection{VEGF in Pathologic Neovascularization: Models of Neovascular AMD}

There is mounting evidence that increased VEGF expression is associated with and causes pathologic neovascularization in AMD and in animal models of AMD (Lopez et al., 1996; Frank et al., 1996; Yi et al., 1997; Baffi et al., 2000; Schwesinger et al., 2001;Grossniklaus et al., 2002; Ishida et al., 2003; Wang et al., 2003). VEGF is found in RPE in surgically excised human AMD specimens of CNV (Lopez et al., 1996; Frank et al., 1996; Grossniklaus et al., 2002). VEGF has been localized to CNV in rodent models of laserinduced CNV (Bora et al., 2005; Yi et al., 1997) and genetically modified mouse models of CNV (Ambati et al., 2003). The overexpression of VEGF in RPE caused intrachoroidal neovascularization in one model (Schwesinger et al., 2001). Recombinant adeno-associated virus-mediated expression of $\mathrm{VEGF}_{165}$ delivered through a subretinal injection caused $\mathrm{CNV}$ (Baffi et al., 2000; Wang et al., 2003).

Controversy exists whether VEGF is sufficient to cause choroidal blood vessels to grow through a normal Bruch's membrane and an intact RPE monolayer, which provides the outer blood-retinal barrier, or whether trauma to the RPE/Bruch's membrane region is additionally necessary for the development of neurosensory retinal CNV. A transgenic mouse model with excessive expression of VEGF in the photoreceptors driven by an inducible promoter system coupled to either the rhodopsin or interphotoreceptor retinoid-binding protein promoter was developed (Ohno-Matsui et al., 2002). These mice showed evidence that CNV could develop in the neurosensory retina without the need for injury to Bruch's membrane or the outer retinal region when excessive VEGF provided a chemotactic gradient in the neurosensory retina. The data also provide in vivo proof of the concept derived from in vitro studies that VEGF plays a role in events important in the development of neurosensory retinal CNV, including a reduction in the RPE barrier properties (Hartnett et al., 2003) that permits a chemotactic gradient of VEGF to form at the apical aspect of the RPE in the photoreceptor region. Once the gradient is formed, activated CECs are attracted to migrate through the RPE into the neurosensory retina (Geisen et al., 2006). Besides local CEC migration, there is also evidence in laser-induced CNV models that bone marrow-derived circulating EPCs home to the lesion site and are involved in CNV development (Takahashi et al., 2004; Espinosa-Heidmann et al., 2003; Csaky et al., 2004). 


\subsection{Genetic Polymorphisms in Untranslated Regions of VEGF}

Various stimuli and factors interplay and lead to the same downstream effect of increased VEGF expression in neovascular AMD (Grossniklaus et al., 2002; Zubilewicz et al., 2001; Kalayoglu et al., 2005). And yet, some patients with strong environmental risk factors for AMD may not manifest the signs. These disparate findings increase the complexity of our understanding of AMD pathogenesis. However, genetic evidence offers a framework to begin to understand some of this complexity. Patients with AMD have been reported to have polymorphisms within the untranslated portions of the VEGF gene (introns and the $5^{\prime}$ UTR) where growth factors and transcription factors may bind to initiate the transcription of VEGF (Churchill et al., 2006). Thus, certain gene mutations within UTR may interfere with binding of different factors to ultimately regulate VEGF transcription. This finding may explain some of the individual genetic variability in the expression of VEGF caused by risk factors and stimuli (Churchill et al., 2006) and the subsequent effects on the development of neovascular forms of AMD.

\subsection{RPE-Extracellular Matrix/Bruch's Membrane}

Bruch's membrane is a five-layered ECM consisting of a central elastin layer bordered by two collagen layers, the outer of these two collagenous layers bordered by the cell membrane of the choriocapillaris endothelium and the inner collagenous layer by the RPE basement membrane. Much evidence points to the importance of changes in Bruch's membrane with age and disease that provide a microenvironment supportive for $\mathrm{CNV}$ development. The association of drusen with vision loss in AMD and with CNV highlighted the importance of changes in ECM components (Bressler et al., 1990; Age-Related Eye Disease Study Research Group, 2005). ECM components in aged or diseased specimens of Bruch's membrane and drusen have been found to be composed of complement and inflammatory fragments (Crabb et al., 2002), oxidized compounds and proteins (Hollyfield et al., 2003), lipid (Sheraidah et al., 1993), lipofuscin (Dorey et al., 1989; Delori et al., 2001), apolipoproteins ( $\mathrm{Li}$ et al., 2006a), and AGE (Hoffmann et al., 2002), to name a few.

\subsection{Stimuli for Neovascular AMD and VEGF Expression}

4.6.1 Inflammation and VEGF expression-The most discussed and accrued evidence linking VEGF, neovascular AMD, and certain components of Bruch's membrane and ECM is that regarding inflammation and inflammatory cytokines. In one study, specimens of CNV obtained during surgery from AMD patients were characterized as inflammatory active (more inflammatory cells than fibrosis) or inactive (more fibrosis than inflammation). VEGF immunoreactivity was greater in inflammatory active CNV and was found in RPE to a greater extent than in macrophages (Grossniklaus et al., 2002). In addition, analyses of human blood for indicators of inflammation, such as C-reactive protein and white blood cell count, showed associations of inflammation with AMD (Seddon et al., 2004; Despriet et al., 2006; Shankar et al., 2007). Finally, a common polymorphism in the complement regulatory protein, complement factor $\mathrm{H}$ (CFH) (Tyr402His), was found to be strongly associated with increased risk of AMD, providing further evidence supporting inflammation in AMD (Klein et al., 2005; Maller et al., 2006; Li et al., 2006b; Haines et al., 2005; Edwards et al., 2005; Rivera et al., 2005).

Other evidence from mouse models and human tissue links complement with VEGF and neovascular AMD. The murine knockout model of Ccl-2, a chemokine important in inflammation and in attracting macrophages, developed drusen, VEGF immunoreactivity, and CNV late in life (Ambati et al., 2003). The investigators proposed that lack of the Ccl-2 chemokine led to reduced recruitment of phagocytosing macrophages and reduced turnover of debris in Bruch's membrane, thus leading to drusen. Furthermore, complement deposition is present in drusen in humans with AMD (Hageman et al., 2001; Crabb et al., 2002). 
Complement factors 3 and 5 (Mullins et al., 2000; Johnson et al., 2000), as well as fragments $\mathrm{C} 3 \mathrm{a}$ and $\mathrm{C} 5 \mathrm{a}$, have been reported as important in AMD and in laser-induced CNV (Nozaki et al., 2006). C3a and C5a caused upregulation of VEGF in RPE in vitro and in vivo (Nozaki et al., 2006). Using C3a and C5a receptor knockout mice, the investigators found reduced macrophage recruitment and reduced CNV after laser injury. In addition, VEGF levels were lower in treated knockout mice compared with treated wild type mice (Nozaki et al., 2006). In other mouse models complement factors $\mathrm{C} 3$ and $\mathrm{B}$ were found essential for the formation of laser-induced CNV (Bora et al., 2006; Bora et al., 2005). In these studies, the end-product of complement activation, the membrane attack complex (MAC), was found to be essential for the development of CNV after laser injury and was temporally associated with VEGF production (Bora et al., 2005). Inhibition of the alternative pathway with small interfering RNA (siRNA) directed against factor B resulted in decreased levels of VEGF, MAC, and $\mathrm{CNV}$, whereas siRNA directed against C1q, a factor involved in the classical pathway, did not affect CNV.

Thus, in susceptible patients, reduced turnover of complement can lead to deposition in Bruch's membrane with drusen formation. Complement can lead to increased VEGF release from RPE and from recruited macrophages (Nozaki et al., 2006), and lead to MAC formation (Bora et al., 2005), which is also temporally associated with CNV and VEGF immunoreactivity in laser-induced CNV models (Fig. 18B). CECs can be activated by VEGF to migrate toward the RPE (Fig. 18C). The confluence of events can lead to forms of neovascular AMD (Fig. 18D). In addition to inflammatory stressors, smoking, an environmental factor shown to greatly increase risk of AMD (Seddon et al., 2006a; Seddon et al., 1996), has been reported to increase serum levels of activated complement factors C3 and B (Robbins et al., 1991; Chao et al., 1982). Thus, stimuli that cause complement accumulation and activation conceivably may stress a system that is not regulated efficiently or effectively and increase the risk of neovascular AMD formation through upregulation of VEGF. This line of thinking may also help explain the late onset of AMD with increasing age and other added stressors throughout life.

4.6.2 Oxidative stress and VEGF expression in AMD-Oxidative stress has been proposed in AMD through several mechanisms (Cai et al., 2000; Spaide et al., 2003), including blue light-induced photochemically released oxidants that damage photoreceptors (Margrain et al., 2004), cigarette smoke-related oxidants such as hydroquinone that alter Bruch's membrane (Espinosa-Heidmann et al., 2006), iron induced oxidative damage to the outer retina (Dunaief, 2006), and possibly through AGE within Bruch's membrane (Tian et al., 2005). Oxidants have been reported to increase the deposition of oxidized proteins or other compounds in Bruch's membrane (Espinosa-Heidmann et al., 2006). In addition, photo-oxidation compounds of lipofuscin (i.e., A2E, a pigment that increases with increasing age) in the outer retina can activate the complement pathways (Zhou et al., 2006) and may lead to CNV and VEGF release as discussed in the previous section. In addition, oxidative compounds have been shown to stimulate RPE to release VEGF. Exposure of RPE to oxidative stress with ter-butyl hydrogen peroxide (Kannan et al., 2006) or by glutathione depletion increased the expression of VEGF, VEGFR-1 and VEGFR-2 (Sreekumar et al., 2006). Treatment of RPE with $\mathrm{H}_{2} \mathrm{O}_{2}$ also led to an increased angiogenic phenotype in CECs (Sreekumar et al., 2006). Furthermore, a murine knockout of the antioxidant enzyme, CuZn superoxide dismutase, developed drusen, CNV and RPE dysfunction, believed to be from disruption in RPE $\beta$-catenin cellular integrity (Imamura et al., 2006).

The hypothesis that oxidative stress causes progression of AMD and vision loss is clinically supported by the Eye Disease Case Control Study (EDCC) that showed reduced AMD (both geographic atrophy and neovascular AMD) in age-matched patients who had higher dietary intake of carotenoids and antioxidants (Eye Disease Case Control Study Group, 1992; Eye 
disease Case Control Group, 1993). The Age-Related Eye Disease Study provided proof that certain antioxidants and zinc supplements, beyond what can be achieved through diet alone, reduced the risk of severe AMD and vision loss in humans (Age-Related Eye Disease Study Research Group, 2001). Further studies are needed to understand possible links of diet, antioxidants and VEGF expression, but there has been evidence that nutritional augmentation can reduce pathologic neovascularization and VEGF expression in the mouse OIR model (Neu et al., 2006). Thus oxidants have been shown to cause increased deposition in Bruch's membrane, which may invoke complement activation, inflammatory mechanisms, and create a pro-angiogenic microenvironment with VEGF release from RPE and recruited macrophages, leading to the development of choroidal neovascularization.

4.6.3 Lipids and VEGF-There is evidence that altered lipid biochemistry can increase the risk of AMD, perhaps by affecting lipid trafficking across Bruch's membrane ( $\mathrm{Li}$ et al., 2006a). Clinically, a number of studies have also reported the association of dietary fats with AMD (Chong et al., 2006). Cholesterol and apoE make up sub-RPE deposits in AMD eyes (Curcio et al., 2005; Li et al., 2006a), and Apo-E4 targeted replacement mice on high fat and cholesterol rich diets developed drusen and CNV (Malek et al., 2005). However, the connection between dietary fat, lipids, and VEGF is less clear. Some evidence is provided by the LDL receptor knockout mice that accumulate lipid in Bruch's membrane and have increased immunoreactivity of VEGF in the RPE, outer plexiform layer, and photoreceptor inner segments, particularly after mice are fed a high fat diet (Rudolf et al., 2005). Also, recombinant Apo-E4 suppressed Ccl-2 and VEGF expression by human RPE and suggested a mechanism by which apolipoproteins can differentially regulate chemokines and VEGF in AMD (Bojanowski et al., 2006).

4.6.4 Extracellular Matrix Proteolysis and VEGF-Proteolytic enzymes are involved in neovascular AMD, and VEGF has been implicated in the signaling pathways of these enzymes (Das and McGuire, 2006). Mice without uPA or plasminogen genes were found to resist laser-induced CNV development (Rakic et al., 2003). Moreover, VEGF induced uPA and tissue-plasminogen activator (tPA) in microvascular endothelial cells (Rakic et al., 2003).

Probably the most studied proteolytic enzymes in AMD are the MMPs, particularly MMP-2 and MMP-9. However, their roles are complex and not fully understood. Generally, MMPs are believed to play a role in remodeling of ECM to facilitate cell migration, release sequestered growth factors, including VEGF, or cause post-translational modification of certain factors (Lambert et al., 2003; Das and McGuire, 2006; Notari et al., 2005). Conversely, VEGF can cause MMP release and activation. VEGF was reported to induce MMP-2 and MMP-9 secretion from RPE in vitro (Hoffmann et al., 2006). In addition, MMP activity was increased in microvascular endothelial cells exposed to VEGF (Lamoreaux et al., 1998). Although some studies suggest an overexpression or activation of MMPs in the pathology of neovascular AMD, others provide evidence that oxidants and toxins increase the formation of sub-RPE deposits and reduce the expression of MMP-2, suggesting that failed ECM turnover leads to deposits within Bruch's membrane and subsequent AMD pathology. Likely, interactions between certain MMPs and their inhibitors (tissue inhibitors of metalloproteinases - TIMPs) are necessary in remodeling ECM in health and are disrupted in injury leading to pathologic events in disease.

A polymorphism in TIMP-3 was found in patients with Sorsby's fundus dystrophy, a hereditary condition that is associated with early development of CNV (Weber et al., 1994), but evidence thus far does not show that TIMP-3 plays a strong role in AMD. However, a binding partner to TIMP-3, known as fibulin 3 or epidermal growth factor-containing fibulin-like extracellular matrix protein 1 (EFEMP-1), was found in Malattia Leventinese, a 
genetic condition associated with a specific appearance of drusen. Overlap between TIMP-3 and EFEMP was found in AMD patients and Malattia Leventinese patients (Klenotic et al., 2004), supporting the role of TIMP-3 in certain groups of patients with AMD. Fibulin 5 expression also was found in eyes with AMD. Polymorphisms in fibulin 5 have been found to account for some AMD cases (Stone et al., 2004). The role of MMPs and TIMPs in AMD is complex. Activation of certain MMPs may be important in turning over Bruch's membrane, whereas excess activity may be implicated in processes initiating angiogenesis, such as enzymatic digestion of ECM prior to EC migration. VEGF can play a role in increasing MMP secretion and activity and also is increased by MMP activation. More evidence supports the notion that complicated interactions of MMPs and TIMPs are important in Bruch's membrane turnover in health and disease.

4.6.5 Aging and AGEs_-Aging leads to the formation of AGE and their receptors (RAGE). RAGE are upregulated in basal deposits from human AMD eyes compared to normal samples (Yamada et al., 2006). AGE have been reported to induce CEC proliferation, VEGF expression and MMP-2 expression in vitro (Hoffmann et al., 2002) and to increase expression of VEGF mRNA from RPE (McFarlane et al., 2005). In addition, AGE-activation of RAGE caused ARPE-19 cells to secrete VEGF by an NFrB-dependent mechanism (McFarlane et al., 2005; Ma et al., 2007). Therefore, a mechanism of RPE release of VEGF may be through RAGE activation after accumulation of AGE with age.

\subsection{Classification of Neovascular AMD: the Importance of RPE-EC Interactions}

Early AMD is classified by clinical features of drusen and associated pigmentary changes. Drusen are subretinal deposits within the RPE basement membrane and Bruch's membrane, and have been shown, as discussed earlier, to be composed of a number of compounds [complement or inflammatory components (Hageman et al., 2001; Crabb et al., 2002), cholesterol and apolipoproteins (Li et al., 2006a), and oxidative modification of lipoproteins, ECM components, TIMP-3, serum albumin, glycoproteins, and crystallins (Nakata et al., 2005; Hollyfield et al., 2003; Yamada et al., 2006)], some of which cause or are associated with an overexpression of VEGF.

Vision loss occurs in the later stages of AMD from geographic atrophy, neovascular AMD, or pigment epithelial detachment (some types considered forms of neovascular AMD). The evidence for VEGF's role is stronger in neovascular AMD than in geographic atrophy.

Neovascular AMD is often considered synonymous with CNV, which includes polypoidal choroidal vasculopathy, a form of neovascular AMD common among Japanese (Sho et al., 2003). However, besides CNV, there are retinal vascular forms of neovascular AMD that are also likely associated with abnormal VEGF expression, since improvement in visual acuity can occur after anti-VEGF therapies (Meyerle et al., 2007). The first described was the retinal vascular abnormality/anomalous complex (RVAC) (Hartnett et al., 1992; Hartnett et al., 1996), in which retinal vessels grew into the deep outer retina and formed leaky angiomatous lesions (Fig. 19A). Vision loss was usually from intra- or subretinal fluid or, at times, from associated pigment epithelial detachments (PED). In some cases, chorioretinal anastomoses occurred in association with PED (Kuhn et al., 1995). Other retinal lesions described later that may represent part of the spectrum of pathology of the RVAC or chorioretinal anastomosis include retinal angiomatous proliferation (RAP) (Yannuzzi et al., 2001) and occult choroidal neovascularization and chorioretinal anastomosis (Gass et al., 2003).

It also is clear from fundus imaging and histopathologic studies (Bressler et al., 1988; Chang et al., 1994; Jalkh et al., 1990; Hartnett and Elsner, 1996), that CNV can develop within different planes: in the sub-RPE space and within Bruch's membrane [occult CNV or ill- 
defined hyperfluorescence on fluorescein angiography (Fig. 19B)]; or within the neurosensory retina above the RPE termed well-defined CNV based on the fluorescein angiographic appearance (Fig. 19C) (Hartnett and Elsner, 1996; Bressler et al., 1990; Macular Photocoagulation Study Group, 1996). In occult CNV, vision can remain stable (Bressler et al., 1988; Macular Photocoagulation Study Group, 1996; Chang et al., 1994) or even improve (Jalkh et al., 1990). However, with time, well-defined areas of hyperfluorescence often develop within occult $\mathrm{CNV}$, suggesting a transition from $\mathrm{CNV}$ beneath the RPE to that within the neurosensory retina. When this occurrs, there is usually a rapid decline in vision (Stevens et al., 1997). Later histopathologic analyses of surgical and ocular specimens show strong correlations between the fundus and fluorescein angiographic appearances and the location of CNV, either above or below the RPE. This led to the classification of CNV lesions as type 1, beneath the RPE, type 2, above the RPE and beneath the neurosensory retina, or a combined form (Grossniklaus and Gass, 1998; Grossniklaus and Green, 2004). The reason why distinction between these types of CNV is important is that occult $\mathrm{CNV}$ can be associated with good vision until the transition to neurosensory retinal CNV occurs (Stevens et al., 1997). Since VEGF is a survival factor for RPE and CECs, inhibition of all its bioactivity may not be desirable. Targeting specific VEGF functions, such as those causing neurosensory retinal CNV to develop from occult CNV, could reduce vision loss from neovascular AMD while retaining VEGF's neuroprotective functions.

\subsection{Heterotypic Cell Interactions and VEGF}

Hartnett and co-workers studied the effect of RPE and endothelial cell interactions on RPE barrier integrity and on CEC transmigration (Hartnett et al., 2003). Co-culture of primary bovine RPE and RECs led to reduced barrier integrity of the RPE as determined by reduced transepithelial electrical resistance (TER) and increased permeability, both measures of barrier properties. Co-culture also increased the release of VEGF into conditioned medium and a neutralizing antibody to VEGF restored TER nearly to baseline in RPE in co-culture with EC (Fig. 20) (Hartnett et al., 2003). VEGF released from ARPE-19 in co-culture with CECs provided a chemotactic gradient to promote CEC transmigration (Geisen et al., 2006) (Fig. 21). CEC transmigration was also increased across RPE-generated ECM compared to fibroblast-generated matrix, providing further evidence that, in addition to soluble VEGF, a cell-associated RPE-generated factor or factors (King et al., 2006) appears to play a role in CEC transmigration (Fig. 22).

Therefore, prior to the development of occult CNV, events occur in the RPE/Bruch's membrane region as described earlier (Fig. 19B). Stresses, such as oxidative compounds and complement deposition and activation in Bruch's membrane, can cause RPE to release VEGF mainly from the basal aspect. VEGF reduces RPE barrier integrity and permits VEGF to enter the neurosensory retinal space, where it can act as a chemoattractant to CECs. CECs are stimulated to migrate toward the RPE. Once proximity or contact of CECs with the RPE or its extracellular matrix occurs (occult CNV, Fig. 18C, Fig. 19B), both soluble and RPE cell-associated isoforms of VEGF are expressed (King et al., 2006). Pathways within CECs, including activation of the small rhoGTPase, Rac1, through PI3K, are activated to facilitate CEC transmigration across the RPE (Peterson et al., 2007) (Fig. 23). CECs migrate into the neurosensory retina and develop areas of neurosensory retinal $\mathrm{CNV}$ that can appear as "breakthrough regions" within occult CNV on fluorescein angiography (Figure 19B). In cases of neurosensory retinal CNV, vision rapidly declines (Stevens et al., 1997). 


\subsection{VEGF to PEDF Ratio}

Pigment epithelium derived factor (PEDF), an inhibitor of angiogenesis and a neuronal survival factor, is preferentially released from the apical aspect of the RPE (Maminishkis et al., 2006) and is postulated to inhibit blood vessel growth originating from either the retinal vasculature or the choroid. Evidence from cell culture and in vivo models of neovascularization provides support that PEDF may be an endogenous negative regulator of VEGF expression and also may interfere with VEGF binding to VEGF receptor 2 (Zhang et al., 2006b). In retinal endothelial cells, VEGF downregulated PEDF (Zhang et al., 2006b). There is also evidence that variability in oxygen-induced angiogenic responses in different strains of mice and rats is related to differences in VEGF and PEDF expression, VEGF lower and PEDF higher in the less angiogenic strains (Chan et al., 2005; Gao et al., 2001). In rats, part of the mechanism appears to be from a mediator upstream from the VEGF gene and promoter (Lu et al., 2007).

Clinically, in eyes from patients without AMD, PEDF mRNA was 10 fold greater than was VEGF mRNA and was also more significantly expressed in peripheral RPE than in macular RPE (Kociok and Joussen, 2007). These data support clinical observations that CNV occurs in the macula much more often than in the peripheral retina. Further evidence that PEDF expression may reduce the likelihood of CNV is from immunohistochemistry of VEGF and PEDF in the RPE and choroid of eyes from AMD and control patients. Specifically, PEDF immunoreactivity was less in RPE/Bruch's membrane/choroid from patients who had had AMD than in age-matched controls (Bhutto et al., 2006). There is also evidence that some stresses, including hypoxia and oxidative stress, two factors associated with neovascular AMD and expression of VEGF, increase the VEGF/PEDF ratio tipping the balance of angiogenic stimulation/inhibition to favor the development of neurosensory retinal CNV. Treatment of RPE with $\mathrm{H}_{2} \mathrm{O}_{2}$ reduced the expression of PEDF mRNA compared to that of vehicle-treated RPE. In addition, the conditioned medium from $\mathrm{H}_{2} \mathrm{O}_{2}$-treated RPE induced microvascular endothelial cell tube formation and migration in vitro in association with an equilibrium shift in VEGF/PEDF ratio (Ohno-Matsui et al., 2001). Hypoxia and VEGF downregulated PEDF via post-translational proteolytic degradation by MMP-2 and MMP-9 (Notari et al., 2005). In human disease, PEDF was deficient in vitreous of patients with CNV compared to age-matched patients undergoing vitrectomy and who did not have AMD (Holekamp et al., 2002; Lambert et al., 2003). Furthermore, excised CNV from eyes that had undergone photodynamic therapy were more likely to have higher immunoreactivity to VEGF and less to PEDF when the lesions had patent vessels and neovascularization than excised CNV with occluded vessels and less neovascularization (Tatar et al., 2006).

Shifting the balance of VEGF/PEDF may be a consideration for treatment of neovascular AMD. However, determining the balance in individual cases may be difficult. Evidence thus far supports PEDF as an anti-angiogenic and neuroprotective agent, and increasing PEDF may be one way to tip the balance in favor of reducing pathologic angiogenesis in AMD.

\subsection{Non-proliferative VEGF Functions in Neovascular AMD}

VEGF has several functions in angiogenesis: proliferation, vasopermeability, chemotaxis, and as a pro-inflammatory growth factor and survival factor. However, its role in neovascular AMD has not been fully determined. From clinical studies using optical coherence tomography (OCT) (Fig. 18), the action of VEGF inhibition using recently approved anti-VEGF treatments appears to be in reducing the subretinal and intraretinal fluid rather than in reducing CNV lesion size (Gragoudas et al., 2004). Through in vitro studies, Hartnett and co-workers found that released VEGF increased permeability in RPE (Hartnett et al., 2003), while Geisen and colleagues found that soluble VEGF increased CEC transmigration but did not cause proliferation of human CECs, even though co-culture- 
conditioned medium from RPE and CECs did (Geisen et al., 2006) (Fig. 24). Furthermore, in vivo studies using laser as an injury to induce CNV in mice, (Nozaki et al., 2006) showed that VEGF, increased prior to laser-induced injury, enhanced CNV volume whereas VEGF, increased after laser-induced injury, reduced CNV volume through activation of VEGFR1 and deactivation of VEGFR2. SPARC (secreted protein, acidic and rich in cysteine) was shown in mouse models to reduce CNV volume, but when laser was performed, the SPARC levels declined (Nozaki et al., 2006), and the decline was postulated to explain the CNV growth by a loss of VEGFR1 activation. The potential role of SPARC in human AMD is currently unknown.

In summary, these studies suggest that neutralizing VEGF activity as a treatment in neovascular AMD may depend on the timing of injury and that its effects may not be to reduce $\mathrm{CEC}$ proliferation as much as to target other angiogenic processes such as vasopermeability, CEC chemotaxis, CEC migration, recruitment of macrophages, and CEC survival. There is, however, concern of the long-term effects on retinal cell survival after broad inhibition of VEGF activity (Blaauwgeers et al., 1999; Witmer et al., 2003; SaintGeniez et al., 2006; Korte et al., 1984; Sakamoto et al., 1995; Hoffmann et al., 2000). Finally, successful treatment of CNV through inhibition of VEGF may depend on the composition of the ECM, and anti-VEGF treatment may at times counter the goal to reduce CNV (Nozaki et al., 2006). Therefore, although inhibition of VEGF activity has improved visual outcomes in patients with neovascular AMD, greater understanding of the molecular events is needed to develop more effective, targeted and safer therapies.

\subsection{Treatment to Block Actions of VEGF}

There are several treatments available that block actions of VEGF, or that are being considered for neovascular AMD (Gupta and Zhang, 2005). In theory, inhibiting VEGF action can be achieved at the gene, mRNA, protein, or receptor level. In general, there are also a number of methods of drug delivery to the posterior segment including those involving intravenous, intravitreous (including slow-release formulations, delivery of nanoparticles, or cells altered through gene therapy) or transscleral (Kao et al., 2005) approaches, sometimes in combination with photodynamic therapy (Fattal and Bochot, 2006). At the time of this writing, FDA approved formulations for AMD include macugen (pegaptanib; OSI Eyetech, Pfizer) and lucentis (ranibizumab; Genentech), both intravitreous injections requiring repeated injections. However, avastin (bevacizumab; Genentech) is approved for systemic use in certain metastatic cancers and has been effectively used as an intravenous or intravitreous agent for human neovascular AMD (Rosenfeld et al., 2005; Michels et al., 2005).

4.11.1 Agents that Reduce VEGF Production-Squalamine is an aminosterol with antineoplastic and antiangiogenic activity and was found to reduce CNV in the laser-induced CNV model in the rat (Ciulla et al., 2003). Rapamycin is a macrolide fungicide that decreases VEGF production and was found to reduce CNV in a laser-induced injury model in mouse (Dejneka et al., 2004).

4.11.2 Agents that Bind VEGF-Some treatment strategies are based on binding VEGF through antibodies (e.g., ranibizumab, bevacizumab). Bevacizumab is a humanized monoclonal antibody that has been approved for systemic use in certain cancers. It was found to be effective in reducing subretinal fluid and improving vision in AMD when given systemically (Michels et al., 2005) or into the eye (Rosenfeld et al., 2006b; Rosenfeld et al., 2005). Systemic administration has side effects including hypertension, proteinuria, and vascular events (Yang et al., 2003). It is interesting to note that the mechanism for preeclampsia, a condition arising during pregnancy and associated with similar systemic effects 
as those resulting from systemic bevacizumab (proteinuria, edema, and hypertension), is believed to be promoted by increased levels of circulating soluble VEGFR-1 (s-Flt-1) (Vuorela et al., 2000), which sequesters VEGF and prevents it from downstream signaling. Also, similar side effects were reported in a study testing another anti-VEGF agent when administered systemically, VEGF Trap (Regeneron). VEGF Trap is a molecule that contains immunoglobulin domains of both VEGFR-1 and VEGFR-2 fused to the constant region of human IgG (Saishin et al., 2003). It functions as a high-affinity soluble receptor that binds and neutralizes both VEGF and placental growth factor. Systemic VEGF Trap reduced CNV volume determined by OCT in a trial of 25 patients (Nguyen et al., 2006). Still, the side effects seen from these clinical trials provide further evidence of the importance of VEGF in normal adult homeostasis. Given intravitreally, bevacizumab has been reported as effective (Costa and Jorge, 2006) and safe (Fung et al., 2006; Maturi et al., 2006). However, some reports of RPE tears (Meyer et al., 2006), and choroidal atrophy in specimens from treated patients (Gibran et al., 2007) raise questions about long-term safety.

Ranibizumab is a smaller $\mathrm{F}(\mathrm{ab})$ fragment of the bevacizumab parent compound and is proposed to have better penetration to the RPE. It has been shown to be effective (Rosenfeld et al., 2006a) and safe and is FDA approved for intravitreal use. Ranibizumab was shown to be effective and superior to photodynamic therapy using verteporfin (Brown et al., 2006). Intravitreous injection of bevacizumab or ranibizumab carries about a $0.03 \%$ risk of side effects with every occurrence (Rosenfeld et al., 2006a). Notably, bevacizumab is approximately 20 -fold less expensive than ranibizumab. A head-to-head trial of bevacizumab and ranibizumab has been approved by NEI/NIH for neovascular AMD.

Macugen was the first drug to be FDA approved for intravitreous use as an injection given every 6 weeks. It is an aptamer that binds preferentially to the heparin binding domain of the VEGF protein (Lee et al., 2005), and is marketed to selectively inhibit the "pathologic" VEGF $_{165}$ isoform (Usui et al., 2004). It was reported to have a significant but modest clinical effect, with loss of three lines of visual acuity or less (defined as a successful outcome) achieved in $70 \%$ of treated, but also in 55\% of uninjected controls (Gragoudas et al., 2004). Further studies testing different treatment regimens are ongoing.

4.11.3 Agents that Inhibit VEGF Receptors-VEGFR-2 has a lower affinity for VEGF than does VEGFR-1, but the receptor tyrosine kinase activity of VEGFR-2 is considered more involved in angiogenic processes (Rahimi, 2006). VEGFR-1 may be involved in angiogenic processes as well when it heterodimerizes with VEGFR-2, but it is also believed to sequester VEGF, preventing it from interacting with VEGFR-2 (Hiratsuka et al., 1998). Inhibitors of the kinase component of VEGFR-2 (i.e., SU5416), sometimes in combination with inhibitors of other receptors including platelet-derived growth factor (PDGF) or stem cell factor, have been tested and found effective in animal models of CNV (Miao et al., 2006; Kinose et al., 2005; Takahashi et al., 2006).

\section{Future Directions}

Our initial experience with pegaptanib and ranibizumab has demonstrated the validity of targeting VEGF in the treatment of ocular angiogenesis. Currently, the use of anti-VEGF therapies to treat increased vascular permeability resulting in macular edema is also being investigated, as are other strategies for VEGF inhibition. RNA interference, for example, is a powerful strategy for silencing the expression of specific genes, and its application to VEGF inhibition for CNV is ongoing (Fattal and Bochot, 2006). Two different short interfering RNA molecules, one directed against VEGF [bevasiranib (Cand5)] and the other against VEGFR-1 (Sirna-027), are currently in clinical trials. Phase I results from VEGFR-1 siRNA showed stabilization of vision in $100 \%$ of patients after a single injection. 
The early success of anti-VEGF therapy for neovascular AMD is certainly encouraging.

However, the effects of long-term VEGF inhibition in the eye will require close monitoring. The constitutive expression of VEGF and its receptors in normal adult neuroretina and choriocapillaris (Kim et al., 1999) suggests that it plays an important role in the survival of neuronal as well as vascular elements, and evidence supporting the neuroprotective effects of VEGF continues to emerge (Storkebaum et al., 2005; Sun and Guo, 2007). There are concerns, for example, that long-term suppression of the survival effects of VEGF may serve to promote geographic atrophy in lieu of neovascular AMD. As well, VEGF is important for stem cell and monocyte/macrophage recruitment, maintenance of kidney and lung barrier function and other non-angiogenic functions (Breen, 2007).

Six full decades after Michaelson first proposed the existence of "factor X", a hypothetical stimulus promoting neovascular retinopathy, anti-VEGF strategies have been adopted and have proven valuable in the treatment of blinding eye conditions. VEGF is only the first of many potential targets, and the continued development of other agents promises future combination therapies that will provide greater and sustained efficacy with less likelihood of toxicity. Additional advances in drug delivery will facilitate long-term intraocular delivery of such molecules, further enabling better and safer treatments.

\section{Acknowledgments}

The authors are indebted to Susan E. Yanni for her contributions to the editing and processing of this review. Its preparation was funded in part by EY007533, EY08126, EY07135 and support from RPB (JSP); EY04618, EY011766, VA Merit Review Award (RBC); EY015130, EY017011 (MEH).

\section{References}

Abu El-Asrar AM, Meersschaert A, et al. Inducible nitric oxide synthase and vascular endothelial growth factor are colocalized in the retinas of human subjects with diabetes. Eye. 2004; 18:306313. [PubMed: 15004583]

Adamis AP, Miller JW, et al. Increased vascular endothelial growth factor levels in the vitreous of eyes with proliferative diabetic retinopathy. Am J Ophthalmol. 1994; 118:445-450. [PubMed: 7943121]

Adamis AP, Shima DT, et al. Synthesis and secretion of vascular permeability factor/vascular endothelial growth factor by human retinal pigment epithelial cells. Biochem Biophys Res Commun. 1993; 193:631-638. [PubMed: 8512562]

Adamis AP, Shima DT, et al. Inhibition of vascular endothelial growth factor prevents retinal ischemia-associated iris neovascularization in a nonhuman primate. Arch Ophthalmol. 1996; 114:66-71. [PubMed: 8540853]

Age-Related Eye Disease Study Research Group. A randomized, placebo-controlled, clinical trial of high-dose supplementation with vitamins $\mathrm{C}$ and $\mathrm{E}$, beta carotene, and zinc for age-related macular degeneration and vision loss: AREDS Report No. 8. Arch Ophthalmol. 2001; 119:1417-1436. [PubMed: 11594942]

Age-Related Eye Disease Study Research Group. A Simplified Severity Scale for Age-Related Macular Degeneration: AREDS Report No. 18. Arch Ophthalmol. 2005; 123:1570-1574. [PubMed: 16286620]

Aiello LP, Avery RL, et al. Vascular endothelial growth factor in ocular fluid of patients with diabetic retinopathy and other retinal disorders. N Engl J Med. 1994; 331:1480-1487. [PubMed: 7526212]

Aiello LP, Davis MD, et al. Effect of ruboxistaurin on visual loss in patients with diabetic retinopathy. Ophthalmology. 2006; 113:2221-2230. [PubMed: 16989901]

Aiello LP, Northrup JM, et al. Hypoxic regulation of vascular endothelial growth factor in retinal cells. Arch Ophthalmol. 1995a; 113:1538-1544. [PubMed: 7487623] 
Aiello LP, Pierce EA, et al. Suppression of retinal neovascularization in vivo by inhibition of vascular endothelial growth factor (VEGF) using soluble VEGF-receptor chimeric proteins. Proc Natl Acad Sci U S A. 1995b; 92:10457-10461. [PubMed: 7479819]

Aiello LP, Gardner TW, et al. Diabetic retinopathy. Diabetes Care. 1998; 21:143-156. [PubMed: 9538986]

Alexandraki K, Piperi C, et al. Inflammatory process in type 2 diabetes: The role of cytokines. Ann N Y Acad Sci. 2006; 1084:89-117. [PubMed: 17151295]

Alon T, Hemo I, et al. Vascular endothelial growth factor acts as a survival factor for newly formed retinal vessels and has implications for retinopathy of prematurity. Nat Med. 1995; 1:1024-1028. [PubMed: 7489357]

Al-Shabrawey, M.; Bartoli, M., et al. Mechanisms of Statin's Protective Actions in Diabetic Retinopathy: Role of NAD(P)H Oxidase and STAT3. submitted

Al-Shabrawey M, Bartoli M, et al. Inhibition of NAD $(\mathrm{P}) \mathrm{H}$ oxidase activity blocks vascular endothelial growth factor overexpression and neovascularization during ischemic retinopathy. Am J Pathol. 2005; 167:599-607. [PubMed: 16049343]

Al-Shabrawey M, Rojas M, et al. Preservation of the Blood Retinal Barrier (BRB) in Diabetes by Deletion of gp91phox or Inhibition of NAD(P)H Oxidase. Invest Ophthalmol Vis Sci. 2006; 47:EAbstract 138.

Ambati J, Anand A, et al. An animal model of age-related macular degeneration in senescent Ccl-2- or Ccr-2-deficient mice. Nat Med. 2003; 9:1390-1397. [PubMed: 14566334]

Anderson CG, Benitz WE, et al. Retinopathy of prematurity and pulse oximetry: a national survey of recent practices. J Perinatol. 2004; 24:164-168. [PubMed: 14999216]

Antonetti DA, Barber AJ, et al. Vascular endothelial growth factor induces rapid phosphorylation of tight junction proteins occludin and zonula occluden 1. A potential mechanism for vascular permeability in diabetic retinopathy and tumors. J Biol Chem. 1999a; 274:23463-23467. [PubMed: 10438525]

Antonetti DA, Barber AJ, et al. Vascular permeability in experimental diabetes is associated with reduced endothelial occludin content: vascular endothelial growth factor decreases occludin in retinal endothelial cells. Penn State Retina Research Group. Diabetes. 1998; 47:1953-1959. [PubMed: 9836530]

Antonetti DA, Lieth E, et al. Molecular mechanisms of vascular permeability in diabetic retinopathy. Semin Ophthalmol. 1999b; 14:240-248. [PubMed: 10758225]

Antonetti DA, Barber AJ, et al. Diabetic retinopathy: seeing beyond glucose-induced microvascular disease. Diabetes. 2006; 55:2401-2411. [PubMed: 16936187]

Asahara T, Murohara T, et al. Isolation of putative progenitor endothelial cells for angiogenesis. Science. 1997; 275:964-967. [PubMed: 9020076]

Ashton N. Neovascularization in ocular disease. Trans Ophthalmol Soc U K. 1961; 81:145-161. [PubMed: 13862868]

Ashton N. Oxygen and the growth and development of retinal vessels. In vivo and in vitro studies The XX Francis I Proctor Lecture. Am J Ophthalmol. 1966; 62:412-435. [PubMed: 5950745]

Ashton N. Donders lecture, 1967 Some aspecrs of the comparative pathology of oxygen toxicity in the retina. Br J Ophthalmol. 1968; 52:505-531. [PubMed: 5724439]

Ashton N. Retinal angiogenesis in the human embryo. Br Med Bull. 1970; 26:103-106. [PubMed: 4911762]

Ashton N, Graymore C, et al. Studies on developing retinal vessels. V Mechanism of vaso-obliteration; a preliminary report. Br J Ophthalmol. 1957; 41:449-460. [PubMed: 13446355]

Askie LM, Henderson-Smart DJ. Restricted versus liberal oxygen exposure for preventing morbidity and mortality in preterm or low birth weight infants. Cochrane Database Syst Rev. 2000:CD001077. [PubMed: 10796409]

Autiero M, Luttun A, et al. Placental growth factor and its receptor, vascular endothelial growth factor receptor-1: novel targets for stimulation of ischemic tissue revascularization and inhibition of angiogenic and inflammatory disorders. J Thromb Haemost. 2003; 1:1356-1370. [PubMed: 12871269]

Prog Retin Eye Res. Author manuscript; available in PMC 2013 June 14. 
Avery RL, Pearlman J, et al. Intravitreal bevacizumab (Avastin) in the treatment of proliferative diabetic retinopathy. Ophthalmology. 2006; 113:1695 e1-15. [PubMed: 17011951]

Baffi J, Byrnes G, et al. Choroidal neovascularization in the rat induced by adenovirus mediated expression of vascular endothelial growth factor. Invest Ophthal Vis Sci. 2000; 41:3582-3589. [PubMed: 11006256]

Bancalari E, Flynn J, et al. Influence of transcutaneous oxygen monitoring on the incidence of retinopathy of prematurity. Pediatrics. 1987; 79:663-669. [PubMed: 3575019]

Barber AJ, Lieth E, et al. Neural apoptosis in the retina during experimental and human diabetes. Early onset and effect of insulin. J Clin Invest. 1998; 102:783-791. [PubMed: 9710447]

Barber AJ, Antonetti DA, et al. Altered expression of retinal occludin and glial fibrillary acidic protein in experimental diabetes. The Penn State Retina Research Group. Invest Ophthalmol Vis Sci. 2000; 41:3561-3568. [PubMed: 11006253]

Barber AJ, Antonetti DA, et al. The Ins2Akita mouse as a model of early retinal complications in diabetes. Invest Ophthalmol Vis Sci. 2005; 46:2210-2218. [PubMed: 15914643]

Bartoli M, Platt D, et al. VEGF differentially activates STAT3 in microvascular endothelial cells. Faseb J. 2003; 17:1562-1564. [PubMed: 12824281]

Bartoli, M.; Lemtalsi, T., et al. Diabetes Induces activation of STAT3 via NAD(P)H oxidasedependent induction of Src kinase. submitted

Bartoli M, Gu X, et al. Vascular Endothelial Growth Factor Activates STAT Proteins in Aortic Endothelial Cells. J Biol Chem. 2000; 275:33189-33192. [PubMed: 10961983]

Bates DO, Curry FE. Vascular endothelial growth factor increases microvascular permeability via a $\mathrm{Ca}(2+)-d e p e n d e n t$ pathway. Am J Physiol. 1997; 273:H687-694. [PubMed: 9277485]

Bates DO, Hillman NJ, et al. Regulation of microvascular permeability by vascular endothelial growth factors. J Anat. 2002; 200:581-597. [PubMed: 12162726]

Behzadian MA, El Remessy AB, et al. Diabetes-induced retinal vascular hyperpermeability requires urokinase receptor (uPAR). 2004; 45:E Abstract 1100.

Behzadian MA, Windsor LJ, et al. VEGF-induced paracellular permeability in cultured endothelial cells involves urokinase and its receptor. Faseb J. 2003; 19:19.

Behzadian MA, Wang XL, et al. TGF-beta increases retinal endothelial cell permeability by increasing MMP-9, possible role of glial cells in endothelial barrier function. Invest Ophthalmol Vis Sci. 2001; 42:853-859. [PubMed: 11222550]

Behzadian MA, Wang XL, et al. Effects of hypoxia on glial cell expression of angiogenesis-regulating factors VEGF and TGF-beta. Glia. 1998; 24:216-225. [PubMed: 9728767]

Benjamin LE, Hemo I. A plasticity window for blood vessel remodelling is defined by pericyte coverage of the preformed endothelial network and is regulated by PDGF-B and VEGF. Development. 1998; 125:1591-1598. [PubMed: 9521897]

Berkowitz BA, Zhang W. Significant reduction of the panretinal oxygenation response after $28 \%$ supplemental oxygen recovery in experimental ROP. Invest Ophthalmol Vis Sci. 2000; 41:192531. [PubMed: 10845618]

Betsholtz C. Insight into the physiological functions of PDGF through genetic studies in mice. Cytokine Growth Factor Rev. 2004; 15:215-228. [PubMed: 15207813]

Bhutto IA, McLeod DS, et al. Pigment epithelium-derived factor (PEDF) and vascular endothelial growth factor (VEGF) in aged human choroid and eyes with age-related macular degeneration. Exp Eye Res. 2006; 82:99-110. [PubMed: 16019000]

Blaauwgeers HGT, Holtkamp GM, et al. Polarized vascular endothelial growth factor secretion by human retinal pigment epithelium and localization of vascular endothelial growth factor receptors on the inner choriocapillaris: evidence for a trophic paracrine relation. Am J Path. 1999; 155:421428. [PubMed: 10433935]

Blanks JC, Johnson LV. Selective lectin binding of the developing mouse retina. J Comp Neurol. 1983; 221:31-41. [PubMed: 6643744]

Blinder KJ, Bradley S, et al. Effect of lesion size, visual acuity, and lesion composition on visual acuity change with and without verteporfin therapy for choroidal neovascularization secondary to age-related macular degeneration: TAP and VIP report no. 1. Am J Ophthalmol. 2003; 136:407418. [PubMed: 12967792] 
Blume-Jensen P, Hunter T. Oncogenic kinase signalling. Nature. 2001; 411:355-365. [PubMed: 11357143]

Bock F, König Y, et al. Inhibition of angiogenesis in the anterior chamber of the eye. Ophthalmologe. 2007; 104:336-344. [PubMed: 17372736]

Boehm BO, Lang G, et al. Low content of the natural ocular anti-angiogenic agent pigment epithelium-derived factor (PEDF) in aqueous humor predicts progression of diabetic retinopathy. Diabetologia. 2003; 46:394-400. [PubMed: 12687338]

Bojanowski CM, Shen D, et al. An apolipoprotein E variant may protect against age-related macular degeneration through cytokine regulation. Environ Mol Mutagen. 2006; 47:594-602. [PubMed: 16823865]

Bora NS, Kaliappan S, et al. Complement Activation via Alternative Pathway Is Critical in the Development of Laser-Induced Choroidal Neovascularization: Role of Factor B and Factor H. J Immunol. 2006; 177:1872-1878. [PubMed: 16849499]

Bora PS, Sohn JH, et al. Role of complement and complement membrane attack complex in laserinduced choroidal neovascularization. J Immunol. 2005; 174:491-497. [PubMed: 15611275]

Bornes S, Boulard M, et al. Control of the vascular endothelial growth factor internal ribosome entry site (IRES) activity and translation initiation by alternatively spliced coding sequences. J Biol Chem. 2004; 279:18717-18726. [PubMed: 14764596]

Boulton ME, Cai J, et al. Gamma-Secretase Regulates Vascular Permeability Through the Association of VEGFR-1 With VE-Cadherin and $\beta$-Catenin in Response to PEDF. Invest Ophthalmol Vis Sci. 2007; 48:E-Abstract 1734.

Breier G, Albrecht U, et al. Expression of vascular endothelial growth factor during embryonic angiogenesis and endothelial cell differentiation. Development. 1992; 114:521-532. [PubMed: 1592003]

Breen EC. VEGF in biological control. J Cell Biochem. 2007; 102:1358-1367. [PubMed: 17979153]

Brennan CM, Steitz JA. HuR and mRNA stability. Cell Mol Life Sci. 2001; 58:266-277. [PubMed: 11289308]

Bressler NM, Frost LA, et al. Natural course of poorly defined choroidal neovascularization associated with macular degeneration. Arch Ophthalmol. 1988; 106:1537-1542. [PubMed: 2461191]

Bressler SB, Maguire MG, et al. Relationship of drusen and abnormalities of the retinal pigment epithelium to the prognosis of neovascular macular degeneration. The Macular Photocoagulation Study Group. Arch Ophthalmol. 1990; 108:1442-1447. [PubMed: 1699513]

Brown DM, Kaiser PK, et al. Ranibizumab versus Verteporfin for Neovascular Age-Related Macular Degeneration. N Engl J Med. 2006; 355:1432-1444. [PubMed: 17021319]

Brown LF, Detmar M, et al. Uterine smooth muscle cells express functional receptors (flt-1 and KDR) for vascular permeability factor/vascular endothelial growth factor. Lab Invest. 1997; 76:245-255. [PubMed: 9042161]

Brown MS, Baron AE, et al. Association between higher cumulative doses of recombinant erythropoietin and risk for retinopathy of prematurity. J AAPOS. 2006; 10:143-149. [PubMed: 16678749]

Brownlee M. Biochemistry and molecular cell biology of diabetic complications. Nature. 2001; 414:813-20. [PubMed: 11742414]

Brownlee M. The pathobiology of diabetic complications: a unifying mechanism. Diabetes. 2005; 54:1615-1625. [PubMed: 15919781]

Bursell SE, Clermont AC, et al. High-dose vitamin E supplementation normalizes retinal blood flow and creatinine clearance in patients with type 1 diabetes. Diabetes Care. 1999; 22:1245-1251. [PubMed: 10480765]

Butler JM, Guthrie SM, et al. SDF-1 is both necessary and sufficient to promote proliferative retinopathy. J Clin Invest. 2005; 115:86-93. [PubMed: 15630447]

Caballero S, Sengupta N, et al. Ischemic vascular damage can be repaired by healthy, but not diabetic, endothelial progenitor cells. Diabetes. 2007; 56:960-967. [PubMed: 17395742]

Cai J, Nelson KC, et al. Oxidative damage and protection of the RPE. Prog Retin Eye Res. 2000; 19:205-221. [PubMed: 10674708] 
Caldwell RB, Bartoli M, et al. Vascular endothelial growth factor and diabetic retinopathy: role of oxidative stress. Curr Drug Targets. 2005; 6:511-24. [PubMed: 16026270]

Campbell FW. Influence of a low atmospheric pressure on the development of the retinal blood vessels. Trans Ophthalmol Soc UK. 1951; 71:287.

Campochiaro P, Nguyen QD, et al. Adenoviral vector-delivered pigment epithelium-derived factor for neovascular age-related macular degeneration: results of a phase I clinical trial. Hum Gene Ther. 2006; 17:167-176. [PubMed: 16454650]

Campochiaro PA. Molecular targets for retinal vascular diseases. J Cell Physiol. 2007; 210:575-581. [PubMed: 17133346]

Campochiaro PA. Retinal and choroidal neovascularization. J Cell Physiol. 2000; 184:301-310. [PubMed: 10911360]

Cao Y, Ji WR, et al. Placenta growth factor: identification and characterization of a novel isoform generated by RNA alternative splicing. Biochem Biophys Res Commun. 1997; 235:493-498. [PubMed: 9207183]

Carmeliet P, Dor Y, et al. Role of HIF-1alpha in hypoxia-mediated apoptosis, cell proliferation and tumour angiogenesis. Nature. 1998; 394(6692):485-490. Erratum in: Nature 1998395 (6701), 525 Keshet E [corrected to Keshert E]. [PubMed: 9697772]

Carmeliet P, Ferreira V, et al. Abnormal blood vessel development and lethality in embryos lacking a single VEGF allele. Nature. 1996; 380:435-439. [PubMed: 8602241]

Ceriello A, Mercuri F, et al. Detection of nitrotyrosine in the diabetic plasma: evidence of oxidative stress. Diabetologia. 2001; 44:834-838. [PubMed: 11508267]

Chan CK, Pham LN, et al. Differential expression of pro- and antiangiogenic factors in mouse straindependent hypoxia-induced retinal neovascularization. Lab Invest. 2005; 85:721-733. [PubMed: 15856049]

Chang TS, Freund KB, et al. Clinicopathologic correlation of choridal neovascularizaiton demonstrated by indocyanine green angiography in a patient with retention of good vision for almost four years. Retina. 1994; 14:114-124. [PubMed: 7518604]

Chan-Ling T, Gock B, et al. The effect of oxygen on vasoformative cell division. Evidence that 'physiological hypoxia' is the stimulus for normal retinal vasculogenesis. Invest Ophthalmol Vis Sci. 1995b; 36:1201-1214. [PubMed: 7775098]

Chan-Ling T, Gock B, et al. Supplemental oxygen therapy. Basis for noninvasive treatment of retinopathy of prematurity. Invest Ophthalmol Vis Sci. 1995a; 36:1215-1230. [PubMed: 7775099]

Chan-Ling T, McLeod DS, et al. Astrocyte-Endothelial Cell Relationships during Human Retinal Vascular Development. Invest Ophthalmol Vis Sci. 2004; 45:2020-2032. [PubMed: 15161871]

Chan-Ling T, Tout S, et al. Vascular changes and their mechanisms in the feline model of retinopathy of prematurity. Invest Ophthalmol Vis Sci. 1992; 33:2128-2147. [PubMed: 1607224]

Chan-Ling TL, Halasz P, et al. Development of retinal vasculature in the cat: processes and mechanisms. Curr Eye Res. 1990; 9:459-478. [PubMed: 2166637]

Chao FC, Tullis JL, et al. Alteration in plasma proteins and platelet functions with aging and cigarette smoking in healthy men. Thromb Haemost. 1982; 47:259-264. [PubMed: 6981228]

Chen CY, Shyu AB. AU-rich elements: characterization and importance in mRNA degradation. Trends Biochem Sci. 1995; 20:465-470. [PubMed: 8578590]

Chen Z, Han ZC. STAT3: A critical transcription activator in angiogenesis. Med Res Rev. 2007

Chong EWT, Sinclair AJ, et al. Facts on fats. Clinical \& Experimental Ophthalmology. 2006; 34:464471. [PubMed: 16872345]

Chow LC, Wright KW, et al. Can changes in clinical practice decrease the incidence of severe retinopathy of prematurity in very low birth weight infants? Pediatrics. 2003; 111:339-345. [PubMed: 12563061]

Churchill AJ, Carter JG, et al. VEGF polymorphisms are associated with neovascular age-related macular degeneration. Hum Mol Genet. 2006; 15:2955-2961. [PubMed: 16940309]

Ciulla TA, Criswell MH, et al. Squalamine lactate reduces choroidal neovascularization in a laserinjury model in the rat. Retina. 2003; 23:808-814. [PubMed: 14707832] 
Clauss M, Weich H, et al. The vascular endothelial growth factor receptor Flt-1 mediates biological activities. Implications for a functional role of placenta growth factor in monocyte activation and chemotaxis. J Biol Chem. 1996; 271:17629-17634. [PubMed: 8663424]

Claxton S, Fruttiger M. Role of arteries in oxygen induced vaso-obliteration. Exp Eye Res. 2003; 77:305-311. [PubMed: 12907163]

Cogan DG. Development and Senescence of the Human Retinal Vasculature. Trans Ophthalmol Soc U K. 1963; 83:465-489. [PubMed: 14123166]

Cohen T, Nahari DLW, et al. Interleukin 6 induces the expression of vascular endothelial growth factor. J Biol Chem. 1996; 271:736-741. [PubMed: 8557680]

Connolly SE, Hores TA, et al. Characterization of vascular development in the mouse retina. Microvasc Res. 1988; 36:275-290. [PubMed: 2466191]

Costa RA, Jorge R. Intravitreal Bevacizumab for Choroidal Neovascularization Caused by AMD (IBeNA Study): Results of a Phase 1 Dose-Escalation Study. Invest Ophthalmol Vis Sci. 2006; 47:4569-4578. [PubMed: 17003454]

Crabb JW, Miyagi M, et al. Drusen proteome analysis: An approach to the etiology of age-related macular degeneration. PNAS. 2002; 99:14682-14687. [PubMed: 12391305]

Cryotherapy for Retinopathy of Prematurity Cooperative Group: Multicenter Trial of Cryotherapy for Retinopathy of Prematurity. Preliminary results. Arch Ophthalmol. 1988; 106:471-479. [PubMed: 2895630]

Csaky KG, Baffi JZ, et al. Recruitment of marrow-derived endothelial cells to experimental choroidal neovascularization by local expression of vascular endothelial growth factor. Exp Eye Res. 2004; 78:1107-1116. [PubMed: 15109917]

Cunningham ET, Adamis AP, et al. A phase II randomized double-masked trial of pegaptanib, an antivascular endothelial growth factor aptamer, for diabetic macular edema. Ophthalmology. 2005; 112:1747-1757. [PubMed: 16154196]

Curcio CA, Presley JB, et al. Esterified and unesterified cholesterol in drusen and basal deposits of eyes with age-related maculopathy. Exp Eye Res. 2005; 81:731-741. [PubMed: 16005869]

Damert A, Ikeda E, et al. Activator-protein-1 binding potentiates the hypoxia-induciblefactor-1mediated hypoxia-induced transcriptional activation of vascular-endothelial growth factor expression in C6 glioma cells. Biochem J. 1997; 327:419-423. [PubMed: 9359410]

D'Amico DJ, Goldberg MF, et al. Anecortave acetate as monotherapy for the treatment of subfoveal lesions in patients with exudative age-related macular degeneration (AMD): interim (month 6) analysis of clinical safety and efficacy. Retina. 2003; 23:14-23. [PubMed: 12652226]

Darland DC, Massingham LJ. Pericyte production of cell-associated VEGF is differentiationdependent and is associated with endothelial survival. Dev Biol. 2003; 264:275-288. [PubMed: 14623248]

Das A, McGuire P. Role of urokinase inhibitors in choroidal neovascularization. Semin Ophthalmol. 2006; 21:23-27. [PubMed: 16517441]

DCCT. The effect of intensive treatment of diabetes on the development and progression of long-term complications in insulin-dependent diabetes mellitus. N Engl J Med. 1993; 329:977-986. [PubMed: 8366922]

de Gooyer TE, Stevenson KA, et al. Retinopathy is reduced during experimental diabetes in a mouse model of outer retinal degeneration. Invest Ophthalmol Vis Sci. 2006; 47:5561-5568. [PubMed: 17122149]

Dejneka NS, Kuroki AM, et al. Systemic rapamycin inhibits retinal and choroidal neovascularization in mice. Mol Vis. 2004; 10:964-972. [PubMed: 15623986]

Delbosc S, Morena M, et al. Statins, 3-hydroxy-3-methylglutaryl coenzyme A reductase inhibitors, are able to reduce superoxide anion production by NADPH oxidase in THP-1-derived monocytes. J Cardiovasc Pharmacol. 2002; 40:611-617. [PubMed: 12352324]

Delori FC, Goger DG, et al. Age-Related Accumulation and Spatial Distribution of Lipofuscin in RPE of Normal Subjects. Invest Ophthalmol Vis Sc. 2001; 42:1855-1866. [PubMed: 11431454]

Deng J, Grande F, et al. Small molecule inhibitors of Stat3 signaling pathway. Curr Cancer Drug Targets. 2007; 7:91-107. [PubMed: 17305481] 
Despriet DD, Klaver CC, et al. Complement factor $\mathrm{H}$ polymorphism, complement activators, and risk of age-related macular degeneration. JAMA. 2006; 296:301-309. [PubMed: 16849663]

Dixelius J, Makinen T, et al. Ligand-induced vascular endothelial growth factor receptor-3 (VEGFR-3) heterodimerization with VEGFR-2 in primary lymphatic endothelial cells regulates tyrosine phosphorylation sites. J Biol Chem. 2003; 278:40973-40979. [PubMed: 12881528]

Dor Y, Porat R, et al. Vascular endothelial growth factor and vascular adjustments to perturbations in oxygen homeostasis. Am J Physiol Cell Physiol. 2001; 280:C1367-1374. [PubMed: 11350731]

Dorey CK, Wu G, et al. Cell loss in the aging retina. Relationship to lipofuscin accumulation and macular degeneration. Invest Ophthalmol Vis Sci. 1989; 30:1691-1699. [PubMed: 2759786]

Dorey CK, Aouididi S, et al. Correlation of vascular permeability factor/vascular endothelial growth factor with extraretinal neovascularization in the rat. Arch Ophthalmol. 1996; 114:1210-1217. [PubMed: 8859080]

Dorrell MI, Aguilar E, et al. Retinal vascular development is mediated by endothelial filopodia, a preexisting astrocytic template and specific R-cadherin adhesion. Invest Ophthalmol Vis Sci. 2002; 43:3500-3510. [PubMed: 12407162]

Dorrell MI, Friedlander M. Mechanisms of endothelial cell guidance and vascular patterning in the developing mouse retina. Prog Retin Eye Res. 2006; 25:277-295. [PubMed: 16515881]

Dreher B, Robinson SR. Development of the retinofugal pathway in birds and mammals: evidence for a common "timetable. Brain Behav Evol. 1988; 31:369-390. [PubMed: 3046710]

Du Y, Miller CM, et al. Hyperglycemia increases mitochondrial superoxide in retina and retinal cells. Free Radic Biol Med. 2003; 35:1491-1499. [PubMed: 14642397]

Du Y, Smith MA, et al. Diabetes-induced nitrative stress in the retina, and correction by aminoguanidine. J Neurochem. 2002; 80:771-779. [PubMed: 11948240]

Dugel PU. Ranibizumab Treatment of Patients With Ocular Diseases. International Ophthalmology Clinics. 2006; 46:131-140. [PubMed: 17060799]

Duh E, Aiello LP. Vascular endothelial growth factor and diabetes: the agonist versus antagonist paradox. Diabetes. 1999; 48:1899-1906. [PubMed: 10512352]

Dunaief JL. Iron Induced Oxidative Damage As a Potential Factor in Age-Related Macular Degeneration: The Cogan Lecture. Invest Ophthalmol Vis Sci. 2006; 47:4660-4664. [PubMed: 17065470]

Edwards AO, Ritter RI, et al. Complement factor $\mathrm{H}$ polymorphism and age-related macular degeneration. Science. 2005; 308:421-424. [PubMed: 15761121]

Eichler W, Yafai Y, et al. Angiogenesis-related factors derived from retinal glial (Muller) cells in hypoxia. Neuroreport. 2004; 15:1633-1637. [PubMed: 15232297]

Ellis EA, Guberski DL, et al. Increased H2O2, vascular endothelial growth factor and receptors in the retina of the BBZ/Wor diabetic rat. Free Radic Biol Med. 2000; 28:91-101. [PubMed: 10656295]

Ellis EA, Grant MB, et al. Increased NADH oxidase activity in the retina of the BBZ/Wor diabetic rat. Free Radic Biol Med. 1998; 24:111-120. [PubMed: 9436620]

El-Remessy A, Abou-Mohamed G, et al. High glucose increases tyrosine nitration and superoxide anion formation in endothelial cells: Role of eNOS uncoupling and aldose reductase activation. Invest Ophthalmol Vis Sci. 2003a; 44:3135-3143. [PubMed: 12824263]

El-Remessy A, Behzadian MA, et al. Peroxynitrite increases vascular permeability in experimental diabetes by a mechanism involving increased expression of VEGF and urokinase plasminogen activator receptor (uPAR). Am J Pathol. 2003b; 162:1995-2004. [PubMed: 12759255]

El-Remessy AB, Al-Shabrawey M, et al. Peroxynitrite mediates VEGF's angiogenic signal and function via a nitration-independent mechanism in endothelial cells. Faseb J. 2007; 21:25282539. [PubMed: 17384142]

El-Remessy AB, Al-Shabrawey M, et al. Neuroprotective and blood-retinal barrier-preserving effects of cannabidiol in experimental diabetes. Am J Pathol. 2006; 168:235-244. [PubMed: 16400026]

El-Remessy AB, Bartoli M, et al. Oxidative stress inactivates VEGF survival signaling in retinal endothelial cells via PI 3-kinase tyrosine nitration. J Cell Sci. 2005; 118:243-252. [PubMed: $15615788]$ 
Ema M, Taya S, et al. A novel bHLH-PAS factor with close sequence similarity to hypoxia-inducible factor 1alpha regulates the VEGF expression and is potentially involved in lung and vascular development. Proc Natl Acad Sci U S A. 1997; 94:4273-4278. [PubMed: 9113979]

Engerman RL, Meyer RK. Development of retinal vasculature in rats. Am J Ophthalmol. 1965; 60:628-641. [PubMed: 5841570]

Espinosa-Heidmann DG, Caicedo A, et al. Bone Marrow-Derived Progenitor Cells Contribute to Experimental Choroidal Neovascularization. Invest Ophthalmol Vis Sci. 2003; 44:4914-4919. [PubMed: 14578417]

Espinosa-Heidmann DG, Suner IJ, et al. Cigarette smoke-related oxidants and the development of subRPE deposits in an experimental animal model of dry AMD. Invest Ophthalmol Vis Sci. 2006; 47:729-737. [PubMed: 16431974]

Esser P, Bresgen M, et al. Intercellular adhesion molecule-1 levels in plasma and vitreous from patients with vitreoretinal disorders. Ger J Ophthalmol. 1995; 4:269-274. [PubMed: 7496336]

Eye disease Case Control Group. Antioxidant status and neovascular age-related macular degeneration. Eye Disease Case-Control Study Group. Arch Ophthalmol. 1993; 111:104-109. [PubMed: 7678730]

Eye Disease Case Control Study Group . Risk factors for neovascular age-related macular degeneration. The Eye Disease Case-Control Study Group. Arch Ophthalmol. 1992; 110:17011708. [PubMed: 1281403]

Fattal E, Bochot A. Ocular delivery of nucleic acids: antisense oligonucleotides, aptamers and siRNA. Adv Drug Deliv Rev. 2006; 58:1203-1223. [PubMed: 17097190]

Feeney SA, Simpson DA, et al. Role of vascular endothelial growth factor and placental growth factors during retinal vascular development and hyaloid regression. Invest Ophthalmol Vis Sci. 2003; 44:839-847. [PubMed: 12556420]

Feng Y, Venema VJ, et al. VEGF-induced permeability increase is mediated by caveolae. Invest Ophthalmol Vis Sci. 1999a; 40:157-167. [PubMed: 9888439]

Feng Y, Venema VJ, et al. VEGF induces nuclear translocation of Flk-1/KDR, endothelial nitric oxide synthase and Caveolin-1 in vascular endothelial cells. Biochem Biophys Res Commun. 1999b; 256:192-197. [PubMed: 10066445]

Ferrara N. Molecular and biological properties of vascular endothelial growth factor. J Mol Med. 1999; 77:527-543. [PubMed: 10494799]

Ferrara N. Role of vascular endothelial growth factor in regulation of physiological angiogenesis. Am J Physiol Cell Physiol. 2001; 280:C1358-1366. [PubMed: 11350730]

Ferrara N. Vascular endothelial growth factor: basic science and clinical progress. Endocr Rev. 2004; 25:581-611. [PubMed: 15294883]

Ferrara N, Carver-Moore K, et al. Heterozygous embryonic lethality induced by targeted inactivation of the VEGF gene. Nature. 1996; 380:439-442. [PubMed: 8602242]

Ferrara N, Davis-Smyth T. The biology of vascular endothelial growth factor. Endocr Rev. 1997; 18:4-25. [PubMed: 9034784]

Ferrara N, Gerber HP, et al. The biology of VEGF and its receptors. Nat Med. 2003; 9:669-676. [PubMed: 12778165]

Flower RW, McLeod DS, et al. Postnatal retinal vascular development of the puppy. Invest Ophthalmol Vis Sci. 1985; 26:957-968. [PubMed: 3159707]

Flynn JT, Bancalari E, et al. Retinopathy of prematurity. A randomized, prospective trial of transcutaneous oxygen monitoring. Ophthalmology. 1987; 94:630-638. [PubMed: 3627711]

Fons P, Herault JP, et al. VEGF-R2 and neuropilin-1 are involved in VEGF-A-induced differentiation of human bone marrow progenitor cells. J Cell Physiol. 2004; 200:351-359. [PubMed: 15254962]

Forstreuter F, Lucius R, et al. Vascular endothelial growth factor induces chemotaxis and proliferation of microglial cells. J Neuroimmunol. 2002; 132:93-98. [PubMed: 12417438]

Forsythe JA, Jiang BH, et al. Activation of vascular endothelial growth factor gene transcription by hypoxia-inducible factor 1. Mol Cell Biol. 1996; 16:4604-4613. [PubMed: 8756616] 
Frank RN, Amin RH, et al. Basic fibroblast growth factor and vascular endothelial growth factor are present in epiretinal and choroidal neovascular membranes. Am J Ophthalmol. 1996; 122:393403. [PubMed: 8794712]

Frank RN. Potential new medical therapies for diabetic retinopathy: protein kinase C inhibitors. Am J Ophthalmol. 2002; 133:693-698. [PubMed: 11992868]

Frank RN. Diabetic retinopathy. N Engl J Med. 2004; 350:48-58. [PubMed: 14702427]

Fruttiger M. Development of the mouse retinal vasculature: angiogenesis versus vasculogenesis. Invest Ophthalmol Vis Sci. 2002; 43:522-527. [PubMed: 11818400]

Fruttiger M. Development of the retinal vasculature. Angiogenesis. 2007; 10:77-88. [PubMed: 17322966]

Fruttiger M, Calver AR, et al. PDGF mediates a neuron-astrocyte interaction in the developing retina. Neuron. 1996; 17:1117-1131. [PubMed: 8982160]

Fuh G, Garcia KC, et al. The interaction of neuropilin-1 with vascular endothelial growth factor and its receptor flt-1. J Biol Chem. 2000; 275:26690-26695. [PubMed: 10842181]

Fujio Y, Walsh K. Akt mediates cytoprotection of endothelial cells by vascular endothelial growth factor in an anchorage-dependent manner. J Biol Chem. 1999; 274:16349-16354. [PubMed: 10347193]

Funatsu H, Yamashita H, et al. Outcome of vitreous surgery and the balance between vascular endothelial growth factor and endostatin. Invest Ophthalmol Vis Sci. 2003; 44:1042-1047. [PubMed: 12601027]

Fung AE, Rosenfeld PJ, et al. The International Intravitreal Bevacizumab Safety Survey: using the internet to assess drug safety worldwide. Br J Ophthalmol. 2006; 90:1344-1349. [PubMed: 16854824]

Gao G, Li Y, et al. Unbalanced expression of VEGF and PEDF in ischemia-induced retinal neovascularization. FEBS Lett. 2001; 489:270-276. [PubMed: 11165263]

Gariano RF. Cellular mechanisms in retinal vascular development. Prog Retin Eye Res. 2003; 22:295306. [PubMed: 12852488]

Gariano RF, Hu D, et al. Expression of angiogenesis-related genes during retinal development. Gene Expr Patterns. 2006; 6:187-92. [PubMed: 16330258]

Gariano RF, Iruela-Arispe ML, et al. Vascular development in primate retina: comparison of laminar plexus formation in monkey and human. Invest Ophthalmol Vis Sci. 1994; 35:3442-3455. [PubMed: 8056520]

Gariano RF, Iruela-Arispe ML, et al. Immunohistochemical characterization of developing and mature primate retinal blood vessels. Invest Ophthalmol Vis Sci. 1996; 37:93-103. [PubMed: 8550339]

Gass JD, Agarwal A, et al. Focal inner retinal hemorrhages in patients with drusen: an early sign of occult choroidal neovascularization and chorioretinal anastomosis. Retina. 2003; 23:741-751. [PubMed: 14707822]

Gavard J, Gutkind JS. VEGF controls endothelial-cell permeability by promoting the beta-arrestindependent endocytosis of VE-cadherin. Nat Cell Biol. 2006; 8:1223-1234. [PubMed: 17060906]

Geisen P, McColm JR, et al. Choroidal endothelial cells transmigrate across the retinal pigment epithelium but do not proliferate in response to soluble vascular endothelial growth factor. Exp Eye Res. 2006; 82:608-619. [PubMed: 16259980]

Gerber HP, McMurtrey A, et al. Vascular endothelial growth factor regulates endothelial cell survival through the phosphatidylinositol 3'-kinase/Akt signal transduction pathway. Requirement for Flk-1/KDR activation. J Biol Chem. 1998b; 273:30336-30343. [PubMed: 9804796]

Gerber HP, Hillan KJ, et al. VEGF is required for growth and survival in neonatal mice. Development. 1999; 126:1149-1159. [PubMed: 10021335]

Gerber HP, Dixit V, et al. Vascular endothelial growth factor induces expression of the antiapoptotic proteins Bcl-2 and A1 in vascular endothelial cells. J Biol Chem. 1998a; 273:13313-13316. [PubMed: 9582377]

Ghirlanda G, Di Leo MA, et al. From functional to microvascular abnormalities in early diabetic retinopathy. Diabetes Metab Rev. 1997; 13:15-35. [PubMed: 9134346] 
Gibran SK, Sachdev A, et al. Histological findings of a choroidal neovascular membrane removed at the time of macular translocation in a patient previously treated with intravitreal bevacizumab treatment (Avastin). Br J Ophthalmol. 2007; 91:602-604. [PubMed: 17166893]

Gilbert RE, Vranes D, et al. Vascular endothelial growth factor and its receptors in control and diabetic rat eyes. Lab Invest. 1998; 78:1017-1027. [PubMed: 9714188]

Gluzman-Poltorak Z, Cohen T, et al. Vascular endothelial growth factor receptor-1 and neuropilin-2 form complexes. J Biol Chem. 2001; 276:18688-18694. [PubMed: 11278319]

Goad DL, Rubin J, et al. Enhanced expression of vascular endothelial growth factor in human SaOS-2 osteoblast-like cells and murine osteoblasts induced by insulin-like growth factor I. Endocrinology. 1996; 137:2262-2268. [PubMed: 8641174]

Gogat K, Le Gat L, et al. VEGF and KDR gene expression during human embryonic and fetal eye development. Invest Ophthalmol Vis Sci. 2004; 45:7-14. [PubMed: 14691147]

Goldberg MA, Schneider TJ. Similarities between the oxygen-sensing mechanisms regulating the expression of vascular endothelial growth factor and erythropoietin. J Biol Chem. 1994; 269:4355-4359. [PubMed: 8308005]

Gole GA, Gannon BJ, et al. Oxygen induced retinopathy: the kitten model reexamined. Aust J Ophthalmol. 1982; 10:223-232. [PubMed: 6186236]

Gordan JD, et al. HIF-2alpha promotes hypoxic cell proliferation by enhancing c-myc transcriptional activity. Cancer Cell. 2007; 11:335-347. [PubMed: 17418410]

Gragoudas ES, Adamis AP, et al. Pegaptanib for neovascular age-related macular degeneration. N Engl J Med. 2004; 351:2805-2816. [PubMed: 15625332]

Graier WF, Posch K, et al. Increased superoxide anion formation in endothelial cells during hyperglycemia: an adaptive response or initial step of vascular dysfunction? Diabetes Res Clin Pract. 1999; 45:153-160. [PubMed: 10588368]

Graier WF, Posch K, et al. Role of superoxide anions in changes of endothelial vasoactive response during acute hyperglycemia. Horm Metab Res. 1997; 29:622-626. [PubMed: 9497899]

Graier WF, Simecek S, et al. High D-glucose-induced changes in endothelial Ca2+/EDRF signaling are due to generation of superoxide anions. Diabetes. 1996; 45:1386-1395. [PubMed: 8826976]

Grant MB, Mames RN, et al. Insulin-like growth factor I as an angiogenic agent. In vivo and in vitro studies. Ann N Y Acad Sci. 1993; 692:230-242. [PubMed: 7692789]

Grant MB, May WS, et al. Adult hematopoietic stem cells provide functional hemangioblast activity during retinal neovascularization. Nat Med. 2002; 8:607-612. [PubMed: 12042812]

Gratton JP, Morales-Ruiz M, et al. Akt down-regulation of p38 signaling provides a novel mechanism of vascular endothelial growth factor-mediated cytoprotection in endothelial cells. J Biol Chem. 2001; 276:30359-30365. [PubMed: 11387313]

Griendling KK, Sorescu D, et al. NAD(P)H oxidase: role in cardiovascular biology and disease. Circ Res. 2000; 86:494-501. [PubMed: 10720409]

Grossniklaus HE, Gass JD. Clinicopathologic correlations of surgically excised type 1 and type 2 submacular choroidal neovascular membranes. Am J Ophthalmol. 1998; 126:59-69. [PubMed: 9683150]

Grossniklaus HE, Green WR. Choroidal neovascularization. Am J Ophthalmol. 2004; 137:496-503. [PubMed: 15013874]

Grossniklaus HE, Ling JX, et al. Macrophage and retinal pigment epithelium expression of angiogenic cytokines in choroidal neovascularization. Mol Vis. 2002; 8:119-126. [PubMed: 11979237]

Gu X, El-Remessy AB, et al. Hyperoxia induces retinal vascular endothelial cell apoptosis through formation of peroxynitrite. Am J Physiol Cell Physiol. 2003; 285:C546-554. [PubMed: 12736139]

Gu X, Samuel S, et al. Effects of sustained hyperoxia on revascularization in experimental retinopathy of prematurity. Invest Ophthalmol Vis Sci. 2002; 43:496-502. [PubMed: 11818396]

Guba M, von Breitenbuch P, et al. Rapamycin inhibits primary and metastatic tumor growth by antiangiogenesis: involvement of vascular endothelial growth factor. Nat Med. 2002; 8:128-135. [PubMed: 11821896] 
Gupta K, Zhang J. Angiogenesis: a curse or cure? Postgrad Med J. 2005; 81:236-242. [PubMed: 15811887]

Guthrie SM, Curtis LM, et al. The nitric oxide pathway modulates hemangioblast activity of adult hematopoietic stem cells. Blood. 2005; 105:1916-1922. [PubMed: 15546953]

Hageman GS, Luthert PJ, et al. An integrated hypothesis that considers drusen as biomarkers of immune-mediated processes at the RPE-Bruch's membrane interface in aging and age-related macular degeneration. Prog Retin Eye Res. 2001; 20:705-732. [PubMed: 11587915]

Haines JL, Hauser MA, et al. Complement factor $\mathrm{H}$ variant increases the risk of age-related macular degeneration. Science. 2005; 308:419-421. [PubMed: 15761120]

Hammes HP, Lin J, et al. Upregulation of the vascular endothelial growth factor/vascular endothelial growth factor receptor system in experimental background diabetic retinopathy of the rat. Diabetes. 1998; 47:401-406. [PubMed: 9519746]

Hammes HP, Lin J, et al. Pericytes and the pathogenesis of diabetic retinopathy. Diabetes. 2002; 51:3107-3112. [PubMed: 12351455]

Han Y, Adams AJ, et al. Multifocal electroretinogram and short-wavelength automated perimetry measures in diabetic eyes with little or no retinopathy. Arch Ophthalmol. 2004; 122:1809-1815. [PubMed: 15596584]

Hanks SK, Quinn AM. Protein kinase catalytic domain sequence database: identification of conserved features of primary structure and classification of family members. Methods Enzymol. 1991; 200:38-62. [PubMed: 1956325]

Harhaj NS, Felinski EA, et al. VEGF activation of protein kinase $\mathrm{C}$ stimulates occludin phosphorylation and contributes to endothelial permeability. Invest Ophthalmol Vis Sci. 2006; 47:5106-5115. [PubMed: 17065532]

Haritoglou C, Kook D, et al. Intravitreal bevacizumab (Avastin) therapy for persistent diffuse diabetic macular edema. Retina. 2006; 26:999-1005. [PubMed: 17151486]

Hartnett ME, Elsner AE. Characteristics of exudative age-related macular degeneration determined in vivo with confocal and indirect infrared imaging. Ophthalmology. 1996; 103:58-71. [PubMed: 8628562]

Hartnett ME, Lappas A, et al. Retinal pigment epithelium and endothelial cell interaction causes retinal pigment epithelial barrier dysfunction via a soluble VEGF-dependent mechanism. Exp Eye Res. 2003; 77:593-599. [PubMed: 14550401]

Hartnett ME, Weiter JJ, et al. Classification of retinal pigment epithelial detachments associated with drusen. Graefes Arch Clin Exper Ophthalmol. 1992; 230:11-19. [PubMed: 1547961]

Hartnett ME, Weiter JJ, et al. Deep retinal vascular anomalous complexes in advanced age-related macular degeneration. Ophthalmology. 1996; 103:2042-2053. [PubMed: 9003338]

Hashimoto T, Zhang XM, et al. VEGF activates divergent intracellular signaling components to regulate retinal progenitor cell proliferation and neuronal differentiation. Development. 2006; 133:2201-2210. [PubMed: 16672338]

Hata Y, Nakagawa K, et al. Hypoxia-induced expression of vascular endothelial growth factor by retinal glial cells promotes in vitro angiogenesis. Virchows Arch. 1995; 426:479-486. [PubMed: 7543339]

$\mathrm{He} \mathrm{H}$, Venema VJ, et al. Vascular endothelial growth factor signals endothelial cell production of nitric oxide and prostacyclin through flk-1/KDR activation of c-Src. J Biol Chem. 1999; 274:25130-25135. [PubMed: 10455194]

Hellberg CB, Boggs SE, et al. Phosphatidylinositol 3-kinase is a target for protein tyrosine nitration. Biochem Biophys Res Commun. 1998; 252:313-317. [PubMed: 9826526]

Hellstrom A, Perruzzi C, et al. Low IGF-I suppresses VEGF-survival signaling in retinal endothelial cells: direct correlation with clinical retinopathy of prematurity. Proc Natl Acad Sci U S A. 2001; 98:5804-5808. [PubMed: 11331770]

Hellstrom A, Engstrom E, et al. Postnatal serum insulin-like growth factor I deficiency is associated with retinopathy of prematurity and other complications of premature birth. Pediatrics. 2003; 112:1016-1020. [PubMed: 14595040] 
Hirano T, Ishihara K, et al. Roles of STAT3 in mediating the cell growth, differentiation and survival signals relayed through the IL-6 family of cytokine receptors. Oncogene. 2000; 19:2548-2556. [PubMed: 10851053]

Hiratsuka S, Minowa O, et al. Flt-1 lacking the tyrosine kinase domain is sufficient for normal development and angiogenesis in mice. PNAS. 1998; 95:9349-9354. [PubMed: 9689083]

Hirschi KK, D'Amore PA. Pericytes in the microvasculature. Cardiovasc Res. 1996; 32:687-698. [PubMed: 8915187]

Hoffmann S, Masood R, et al. Selective killing of RPE with a vascular endothelial growth factor chimeric toxin. Invest Ophthalmol Vis Sci. 2000; 41:2389-2393. [PubMed: 10892888]

Hoffmann S, He S, et al. MMP-2 and MMP-9 secretion by rpe is stimulated by angiogenic molecules found in choroidal neovascular membranes. Retina. 2006; 26:454-461. [PubMed: 16603966]

Hoffmann S, Friedrichs U, et al. Advanced glycation end products induce choroidal endothelial cell proliferation, matrix metalloproteinase-2 and VEGF upregulation in vitro. Graefes Arch Clin Exp Ophthalmol. 2002; 240:996-1002. [PubMed: 12483322]

Holash JA, Stewart PA. The relationship of astrocyte-like cells to the vessels that contribute to the blood-ocular barriers. Brain Res. 1993; 629:218-224. [PubMed: 7906600]

Holekamp NM, Bouck N, et al. Pigment epithelium-derived factor is deficient in the vitreous of patients with choroidal neovascularization due to age-related macular degeneration. Am J Ophthalmol. 2002; 134:220-227. [PubMed: 12140029]

Hollborn M, Tenckhoff S, et al. Human retinal epithelium produces and responds to placenta growth factor. Graefes Arch Clin Exp Ophthalmol. 2006; 244:732-741. [PubMed: 16341703]

Hollyfield JG, Salomon RG, et al. Proteomic approaches to understanding age-related macular degeneration. Adv Exp Med Biol. 2003; 533:83-89. [PubMed: 15180251]

Holmqvist K, Cross MJ, et al. The adaptor protein shb binds to tyrosine 1175 in vascular endothelial growth factor (VEGF) receptor-2 and regulates VEGF-dependent cellular migration. J Biol Chem. 2004; 279:22267-22275. [PubMed: 15026417]

Hong KH, Ryu J, et al. Monocyte chemoattractant protein-1-induced angiogenesis is mediated by vascular endothelial growth factor-A. Blood. 2005; 105:1405-1407. [PubMed: 15498848]

Horiuchi M, Cui TX, et al. Fluvastatin enhances the inhibitory effects of a selective angiotensin II type 1 receptor blocker, valsartan, on vascular neointimal formation. Circulation. 2003; 107:106-112. [PubMed: 12515751]

Hu CJ, Wang LY, et al. Differential roles of hypoxia-inducible factor 1alpha (HIF-1alpha) and HIF-2alpha in hypoxic gene regulation. Mol Cell Biol. 2003; 23:9361-9374. [PubMed: 14645546]

Hudson CC, Liu M, et al. Regulation of hypoxia-inducible factor 1alpha expression and function by the mammalian target of rapamycin. Mol Cell Biol. 2002; 22:7004-7014. [PubMed: 12242281]

Huez I, Bornes S, et al. New vascular endothelial growth factor isoform generated by internal ribosome entry site-driven CUG translation initiation. Mol Endocrinol. 2001; 15:2197-2210. [PubMed: 11731620]

Hughes S, Yang H, et al. Vascularization of the human fetal retina: roles of vasculogenesis and angiogenesis. Invest Ophthalmol Vis Sci. 2000; 41:1217-1228. [PubMed: 10752963]

Ida $\mathrm{H}$, Tobe $\mathrm{T}$, et al. RPE cells modulate subretinal neovascularization, but do not cause regression in mice with sustained expression of VEGF. Invest Ophthalmol Vis Sci. 2003; 44:5430-5437. [PubMed: 14638748]

Ideno J, Mizukami H, et al. Prevention of diabetic retinopathy by intraocular soluble flt-1 gene transfer in a spontaneously diabetic rat model. Int J Mol Med. 2007; 19:75-79. [PubMed: 17143550]

Igarashi K, Shigeta K, et al. Sck interacts with KDR and Flt-1 via its SH2 domain. Biochem Biophys Res Comm. 1998; 251:77-82. [PubMed: 9790910]

Ikeda E, Achen MG, et al. Hypoxia-induced transcriptional activation and increased mRNA stability of vascular endothelial growth factor in C6 glioma cells. J Biol Chem. 1995; 270 (34):1976119766. [PubMed: 7544346]

Imamura Y, Noda S, et al. Drusen, choroidal neovascularization, and retinal pigment epithelium dysfunction in SOD1-deficient mice: A model of age-related macular degeneration. PNAS. 2006; 103:11282-11287. [PubMed: 16844785] 
Inoguchi T, Sonta $\mathrm{T}$, et al. Protein kinase $\mathrm{C}$-dependent increase in reactive oxygen species (ROS) production in vascular tissues of diabetes: role of vascular NAD(P)H oxidase. J Am Soc Nephrol. 2003a; 14:S227-232. [PubMed: 12874436]

Inoguchi $\mathrm{T}$, Tsubouchi $\mathrm{H}$, et al. A possible target of antioxidative therapy for diabetic vascular complications-vascular NAD(P)H oxidase. Curr Med Chem. 2003b; 10:1759-1764. [PubMed: 12871120]

Ishida S, Usui T, et al. VEGF164 is proinflammatory in the diabetic retina. Invest Ophthalmol Vis Sci. 2003; 44:2155-2162. [PubMed: 12714656]

Issbrucker K, Marti HH, et al. p38 MAP kinase - a molecular switch between VEGF-induced angiogenesis and vascular hyperpermeability. FASEB J. 2003; 17:262-264. [PubMed: 12490545]

Iyer NV, Kotch LE, et al. Cellular and developmental control of $\mathrm{O} 2$ homeostasis by hypoxia-inducible factor 1 alpha. Genes Dev. 1998; 12:149-162. [PubMed: 9436976]

Jain SK. Superoxide dismutase overexpression and cellular oxidative damage in diabetes. A commentary on "Overexpression of mitochondrial superoxide dismutase in mice protects the retina from diabetes-induced oxidative stress”. Free Radic Biol Med. 2006; 41:1187-1190. [PubMed: 17015164]

Jakeman LB, Armanini M, et al. Developmental expression of binding sites and messenger ribonucleic acid for vascular endothelial growth factor suggests a role for this protein in vasculogenesis and angiogenesis. Endocrinology. 1993; 133:848-859. [PubMed: 7688292]

Jalkh AE, Nasrallah FP, et al. Inactive subretinal neovascularization in age-related macular degeneration. Ophthalmology. 1990; 97:1614-1618. [PubMed: 2087293]

Jiang B, Bezhadian MA, et al. Astrocytes modulate retinal vasculogenesis: effects on endothelial cell differentiation. Glia. 1995; 15:1-10. [PubMed: 8847096]

Jiang B, Liou GI, et al. Astrocytes modulate retinal vasculogenesis: effects on fibronectin expression. J Cell Sci. 1994; 107:2499-2508. [PubMed: 7844167]

Johnson LV, Ozaki S, et al. A Potential Role for Immune Complex Pathogenesis in Drusen Formation. Exp Eye Res. 2000; 70:441-449. [PubMed: 10865992]

Jorge R, Costa RA, et al. Intravitreal bevacizumab (Avastin) for persistent new vessels in diabetic retinopathy (IBEPE study). Retina. 2006; 26:1006-1013. [PubMed: 17151487]

Joussen AM, Murata T, et al. Leukocyte-mediated endothelial cell injury and death in the diabetic retina. Am J Pathol. 2001; 158:147-152. [PubMed: 11141487]

Joussen AM, Poulaki V, et al. A central role for inflammation in the pathogenesis of diabetic retinopathy. Faseb J. 2004; 18:1450-1452. [PubMed: 15231732]

Joussen AM, Poulaki V, et al. Retinal vascular endothelial growth factor induces intercellular adhesion molecule-1 and endothelial nitric oxide synthase expression and initiates early diabetic retinal leukocyte adhesion in vivo. Am J Pathol. 2002; 160:501-509. [PubMed: 11839570]

Jussila L, Alitalo K. Vascular growth factors and lymphangiogenesis. Physiol Rev. 2000; 82:673-700. [PubMed: 12087132]

Juul SE, Anderson DK, et al. Erythropoietin and erythropoietin receptor in the developing human central nervous system. Pediatr Res. 1998; 43:40-49. [PubMed: 9432111]

Kalayoglu MV, Bula D, et al. Identification of Chlamydia pneumoniae within human choroidal neovascular membranes secondary to age-related macular degeneration. Graefes Arch Clin Exp Ophthalmol. 2005; 243:1080-1090. [PubMed: 15909160]

Kannan R, Zhang N, et al. Stimulation of apical and basolateral VEGF-A and VEGF-C secretion by oxidative stress in polarized retinal pigment epithelial cells. Mol Vis. 2006; 12:1649-1659. [PubMed: 17200665]

Kao JC, Geroski DH, et al. Transscleral permeability of fluorescent-labeled antibiotics. J Ocul Pharmacol Ther. 2005; 21:1-10. [PubMed: 15718823]

Karpanen T, Heckman CA, et al. Functional interaction of VEGF-C and VEGF-D with neuropilin receptors. FASEB J. 2006; 20:1462-1472. [PubMed: 16816121]

Keck PJ, Hauser SD, et al. Vascular permeability factor, an endothelial cell mitogen related to PDGF. Science. 1989; 246:1309-1312. [PubMed: 2479987] 
Kelly DJ, Zhang Y, et al. Cells expressing the stem cell factor receptor, c-kit, contribute to neoangiogenesis in diabetes. Diab Vasc Dis Res. 2005; 2:76-80. [PubMed: 16305063]

Kent D, Vinores SA, et al. Macular oedema: the role of soluble mediators. Br J Ophthalmol. 2000; 84:542-545. [PubMed: 10781524]

Keyt BA, Berleau LT, et al. Identification of vascular endothelial growth factor determinants for binding KDR and FLT-1 receptors. Generation of receptor-selective VEGF variants by sitedirected mutagenesis. J Biol Chem. 1996; 271:7788-7795. [PubMed: 8631822]

Kim I, Ryan AM, et al. Constitutive expression of VEGF, VEGFR-1, and VEGFR-2 in normal eyes. Invest Ophthalmol Vis Sci. 1999; 40:2115-2121. [PubMed: 10440268]

Kimura S, Zhang GX, et al. Role of NAD(P)H oxidase- and mitochondria-derived reactive oxygen species in cardioprotection of ischemic reperfusion injury by angiotensin II. Hypertension. 2005; 45:860-866. [PubMed: 15824196]

King B, Geisen P, et al. The role of cell-associated VEGF isoforms on the transmigration of choroidal endothelial cells across the retinal pigment epithelium. Invest Ophthalmol Vis Sci. 2006 ARVO abstract.

King GL, Goodman AD, et al. Receptors and growth-promoting effects of insulin and insulinlike growth factors on cells from bovine retinal capillaries and aorta. J Clin Invest. 1985; 75:10281036. [PubMed: 2984251]

Kinose F, Roscilli G, et al. Inhibition of retinal and choroidal neovascularization by a novel KDR kinase inhibitor. Mol Vis. 2005; 11:366-373. [PubMed: 15951738]

Kinsey VE, Arnold $\mathrm{HJ}$, et al. $\mathrm{PaO} 2$ levels and retrolental fibroplasia: a report of the cooperative study. Pediatrics. 1977; 60:655-668. [PubMed: 578921]

Klampfer L. Signal transducers and activators of transcription (STATs): Novel targets of chemopreventive and chemotherapeutic drugs. Curr Cancer Drug Targets. 2006; 6:107-121. [PubMed: 16529541]

Klein RJ, Zeiss C, et al. Complement factor $\mathrm{H}$ polymorphism in age-related macular degeneration. Science. 2005; 308:385-389. [PubMed: 15761122]

Klein R, Klein BE, et al. The Wisconsin epidemiologic study of diabetic retinopathy. II Prevalence and risk of diabetic retinopathy when age at diagnosis is less than 30 years. Arch Ophthalmol. 1984; 102:520-526. [PubMed: 6367724]

Klein R, Klein BE. Diabetic eye disease. Lancet. 1997; 350:197-204. [PubMed: 9250197]

Klein, R.; Klein, BE. Diabetes in America. In: Harris, MI., editor. National Institutes of health, National Institute of Diabetes and Digestive and Kidney diseases. 2. US public Health Service; 1995. (NIH Publ no. 95-1468)

Klenotic PA, Munier FL, et al. Tissue Inhibitor of Metalloproteinases-3 (TIMP-3) Is a Binding Partner of Epithelial Growth Factor-containing Fibulin-like Extracellular Matrix Protein 1 (EFEMP1): IMPLICATIONS FOR MACULAR DEGENERATIONS. J Biol Chem. 2004; 279:30469_ 30473. [PubMed: 15123717]

Kociok N, Joussen AM. Varied expression of functionally important genes of RPE and choroid in the macula and in the periphery of normal human eyes. Graefes Arch Clin Exp Ophthalmol. 2007; 245:101-113. [PubMed: 16598467]

Konopatskaya O, Churchill AJ, et al. VEGF165b, an endogenous C-terminal splice variant of VEGF, inhibits retinal neovascularization in mice. Mol Vis. 2006; 12:626-632. [PubMed: 16735996]

Korte GE, Reppucci V, et al. RPE destruction causes choriocapillary atrophy. Invest Ophthalmol Vis Sci. 1984; 25:1135-1145. [PubMed: 6480292]

Kowluru RA. Effect of reinstitution of good glycemic control on retinal oxidative stress and nitrative stress in diabetic rats. Diabetes. 2003; 52:818-23. [PubMed: 12606525]

Kowluru RA, Tang J, et al. Abnormalities of retinal metabolism in diabetes and experimental galactosemia. VII Effect of long-term administration of antioxidants on the development of retinopathy. Diabetes. 2001; 50:1938-1942. [PubMed: 11473058]

Kowluru RA, Koppolu P. Termination of experimental galactosemia in rats, and progression of retinal metabolic abnormalities. Invest Ophthalmol Vis Sci. 2002; 43:3287-3291. [PubMed: 12356836] 
Kowluru RA, Engerman RL, et al. Abnormalities of retinal metabolism in diabetes or experimental galactosemia VIII. Prevention by aminoguanidine. Curr Eye Res. 2000; 21:814-819. [PubMed: 11120572]

Kowluru RA, Odenbach S. Effect of long-term administration of alpha-lipoic acid on retinal capillary cell death and the development of retinopathy in diabetic rats. Diabetes. 2004; 53:3233-3238. [PubMed: 15561955]

Krantz SB. Erythropoietin. Blood. 1991; 77:419-434. [PubMed: 1991159]

Kretzer FL, Hittner HM. Spindle cells and retinopathy of prematurity: interpretations and predictions. Birth Defects Orig Artic Ser. 1988; 24:147-168. [PubMed: 3052608]

Kuhn D, Meunier I, et al. Imaging of chorioretinal anastomoses in vascularized retinal pigment epithelium detachments. Arch Ophthalmol. 1995; 113:1392-1398. [PubMed: 7487600]

Kunisaki M, Bursell SE, et al. Vitamin E prevents diabetes-induced abnormal retinal blood flow via the diacylglycerol-protein kinase C pathway. Am J Physiol. 1995; 269:E239-246. [PubMed: 7653541]

Kuroki M, Voest EE, et al. Reactive oxygen intermediates increase vascular endothelial growth factor expression in vitro and in vivo. J Clin Invest. 1996; 98:1667-1675. [PubMed: 8833917]

Ladomery MR, Harper SJ, et al. Alternative splicing in angiogenesis: the vascular endothelial growth factor paradigm. Cancer Lett. 2007; 249:133-142. [PubMed: 17027147]

Lamalice L, Houle F, et al. Phosphorylation of tyrosine 1214 on VEGFR2 is required for VEGFinduced activation of Cdc42 upstream of SAPK2/p38. Oncogene. 2004; 23:434-445. [PubMed: 14724572]

Lambert V, Wielockx B, et al. MMP-2 and MMP-9 synergize in promoting choroidal neovascularization. FASEB J. 2003; 17:2290-2292. [PubMed: 14563686]

Lamoreaux WJ, Fitzgerald MEC, et al. Vascular endothelial growth factor increases release of gelatinase A and decreases release of tissue inhibitor of metalloproteinases by microvascular endothelial cells in vitro. Microvasc Res. 1998; 55:29-42. [PubMed: 9473407]

Laughner E, Taghavi P, et al. HER2 (neu) signaling increases the rate of hypoxia-inducible factor 1alpha (HIF-1alpha) synthesis: novel mechanism for HIF-1-mediated vascular endothelial growth factor expression. Mol Cell Biol. 2001; 21:3995-4004. [PubMed: 11359907]

Leaf AA, Green CR, et al. Maturation of electroretinograms and visual evoked potentials in preterm infants. Dev Med Child Neurol. 1995; 37:814-826. [PubMed: 7589864]

Lee JH, Canny MD, et al. A therapeutic aptamer inhibits angiogenesis by specifically targeting the heparin binding domain of VEGF165. PNAS. 2005; 102:18902-18907. [PubMed: 16357200]

Lee IG, Chae SL, et al. Involvement of circulating endothelial progenitor cells and vasculogenic factors in the pathogenesis of diabetic retinopathy. Eye. 2006a; 20:546-552. [PubMed: 15905870]

Lee SB, Bae IH, et al. Link between mitochondria and NADPH oxidase 1 isozyme for the sustained production of reactive oxygen species and cell death. J Biol Chem. 2006b; 281:36228-36235. [PubMed: 17015444]

Levy AP, Levy NS, et al. Regulation of vascular endothelial growth factor in cardiac myocytes. Circ Res. 1995; 76:758-766. [PubMed: 7728992]

Levy AP, Levy NS, et al. Post-transcriptional regulation of vascular endothelial growth factor by hypoxia. J Biol Chem. 1996; 271:2746-2753. [PubMed: 8576250]

Levy NS, Chung S, et al. Hypoxic stabilization of vascular endothelial growth factor mRNA by the RNA-binding protein HuR. J Biol Chem. 1998; 273:6417-6423. [PubMed: 9497373]

Li CM, Clark ME, et al. Apolipoprotein Localization in Isolated Drusen and Retinal Apolipoprotein Gene Expression. Invest Ophthalmol Vis Sci. 2006a; 47:3119-3128. [PubMed: 16799058]

Li M, Atmaca-Sonmez P, et al. CFH haplotypes without the Y402H coding variant show strong association with susceptibility to age-related macular degeneration. Nat Genet. 2006b; 38:10491054. [PubMed: 16936733]

Li J, Perrella MA, et al. Induction of vascular endothelial growth factor gene expression by interleukin-1 beta in rat aortic smooth muscle cells. J Biol Chem. 1995; 270:308-312. [PubMed: 7814392] 
Liao JK. Beyond lipid lowering: the role of statins in vascular protection. Int J Cardiol. 2002; 86:5-18. [PubMed: 12243846]

Lindahl P, Johansson BR, et al. Pericyte loss and microaneurysm formation in PDGF-B-deficient mice. Science. 1997; 277:242-245. [PubMed: 9211853]

Ling TL, Stone J. The development of astrocytes in the cat retina: evidence of migration from the optic nerve. Brain Res Dev Brain Res. 1988; 44:73-85.

Lopez PF, Sippy BD, et al. Transdifferentiated retinal pigment epithelial cells are immunoreactive for vascular endothelial growth factor in surgically excised age-related macular degeneration-related choroidal neovascular membranes. Invest Ophthalmol Vis Sci. 1996; 37:855-868. [PubMed: 8603870]

Lu K, Zhou Y, et al. Rat strain-dependent susceptibility to ischemia-induced retinopathy associated with retinal vascular endothelial growth factor regulation. J Mol Endocrinol. 2007; 38:423-432. [PubMed: 17446232]

Lu M, Perez VL, et al. VEGF increases retinal vascular ICAM-1 expression in vivo. Invest Ophthalmol Vis Sci. 1999; 40:1808-1812. [PubMed: 10393052]

Lucey JF, Dangman B. A reexamination of the role of oxygen in retrolental fibroplasia. Pediatrics. 1984; 73:82-96. [PubMed: 6419199]

Lutty GA, Cao J, et al. Relationship of polymorphonuclear leukocytes to capillary dropout in the human diabetic choroid. Am J Pathol. 1997; 151:707-714. [PubMed: 9284819]

Lutty GA, McLeod DS. A new technique for visualization of the human retinal vasculature. Arch Ophthalmol. 1992; 110:267-276. [PubMed: 1736877]

Ma W, Lee SE, et al. RAGE Ligand Upregulation of VEGF Secretion in ARPE-19 Cells. Invest Ophthalmol Vis Sci. 2007; 48:1355-1361. [PubMed: 17325184]

Macular Photocoagulation Study Group. Occult choroidal neovascularization: Influence on visual outcome in patients with age-related macular degeneration. Arch Ophthalmol. 1996; 114:400411. [PubMed: 8602776]

Madan A, Penn JS. Animal models of oxygen-induced retinopathy. Front Biosci. 2003; 8:d1030-1043. [PubMed: 12700061]

Maharaj ASR, Saint-Geniez M, et al. Vascular Endothelial Growth Factor Localization in the Adult. Am J Path. 2006; 168:639-648. [PubMed: 16436677]

Maisonpierre PC, Suri C, et al. Angiopoietin-2, a natural antagonist for Tie2 that disrupts in vivo angiogenesis. Science. 1997; 277:55-60. [PubMed: 9204896]

Makowski GS, Ramsby ML. Binding of latent matrix metalloproteinase 9 to fibrin: activation via a plasmin-dependent pathway. Inflammation. 1998; 22:287-305. [PubMed: 9604716]

Malek G, Johnson LV, et al. Apolipoprotein E allele-dependent pathogenesis: A model for age-related retinal degeneration. PNAS. 2005; 102:11900-11905. [PubMed: 16079201]

Maller J, George S, et al. Common variation in three genes, including a noncoding variant in $\mathrm{CFH}$, strongly influences risk of age-related macular degeneration. Nat Genet. 2006; 38:1055-1059. [PubMed: 16936732]

Maminishkis A, Chen S, et al. Confluent monolayers of cultured human fetal retinal pigment epithelium (hfRPE) exhibit morphology and physiology of native tissue. Invest Ophthalmol Vis Sci. 2006; 47:3612-3624. [PubMed: 16877436]

Mandriota SJ, Seghezzi G, et al. Vascular endothelial growth factor increases urokinase receptor expression in vascular endothelial cells. J Biol Chem. 1995; 270:9709-9716. [PubMed: 7730348]

Marcus DM, Peskin E. The age-related macular degeneration radiotherapy trial (AMDRT): One year results from a pilot study. Am J Ophthalmol. 2004; 138:818-828. [PubMed: 15531318]

Margrain TH, Boulton M, et al. Do blue light filters confer protection against age-related macular degeneration? Prog Retin Eye Res. 2004; 23:523-531. [PubMed: 15302349]

Marneros AG, Fan J, et al. Vascular Endothelial Growth Factor Expression in the Retinal Pigment Epithelium Is Essential for Choriocapillaris Development and Visual Function. Am J Path. 2005; 167:1451-1459. [PubMed: 16251428] 
Matsumoto K, Sera Y, et al. Serum concentrations of soluble adhesion molecules are related to degree of hyperglycemia and insulin resistance in patients with type 2 diabetes mellitus. Diabetes Res Clin Pract. 2002; 55:131-138. [PubMed: 11796179]

Matsumoto T, Bohman S, et al. VEGF receptor-2 Y951 signaling and a role for the adapter molecule TSAd in tumor angiogenesis. EMBO J. 2005; 24:2342-2353. [PubMed: 15962004]

Matsumoto T, Turesson I, et al. p38 MAP kinase negatively regulates endothelial cell survival, proliferation, and differentiation in FGF-2-stimulated angiogenesis. J Cell Biol. 2002; 156:149_ 160. [PubMed: 11781339]

Maturi RK, Bleau LA, et al. Electrophysiologic findings after intravitreal bevacizumab (Avastin) treatment. Retina. 2006; 26:270-274. [PubMed: 16508425]

McColm JR, Geisen P, et al. VEGF isoforms and their expression after a single episode of hypoxia or repeated fluctuations between hyperoxia and hypoxia: relevance to clinical ROP. Mol Vis. 2004; 21:512-520. [PubMed: 15303088]

McFarlane S, Glenn JV, et al. Characterisation of the advanced glycation endproduct receptor complex in the retinal pigment epithelium. Br J Ophthalmol. 2005; 89:107-112. [PubMed: 15615757]

McLeod DS, D'Anna SA, et al. Clinical and histopathologic features of canine oxygen-induced proliferative retinopathy. Invest Ophthalmol Vis Sci. 1998; 39:1918-1932. [PubMed: 9727415]

McLeod DS, Hasegawa T. The initial fetal human retinal vasculature develops by vasculogenesis. Dev Dyn. 2006; 235 (12):3336-3347. [PubMed: 17061263]

McLeod DS, Lutty GA, et al. Visualization of a developing vasculature. Microvasc Res. 1987; 33:257-269. [PubMed: 2438539]

McMullen ME, Bryant PW, et al. Activation of p38 has opposing effects on the proliferation and migration of endothelial cells. J Biol Chem. 2005; 280:20995-21003. [PubMed: 15790570]

Melder RJ, Koenig GC, et al. During angiogenesis, vascular endothelial growth factor and basic fibroblast growth factor regulate natural killer cell adhesion to tumor endothelium. Nat Med. 1996; 2:992-997. [PubMed: 8782456]

Meleth AD, Agron E, et al. Serum inflammatory markers in diabetic retinopathy. Invest Ophthalmol Vis Sci. 2005; 46:4295-4301. [PubMed: 16249511]

Meyer CH, Mennel S, et al. Acute retinal pigment epithelial tear following intravitreal bevacizumab (Avastin) injection for occult choroidal neovascularisation secondary to age related macular degeneration. Br J Ophthalmol. 2006; 90:1207-1208. [PubMed: 16929069]

Meyerle CB, Freund KB, et al. Intravitreal bevacizumab (avastin) for retinal angiomatous proliferation. Retina. 2007; 27:451-457. [PubMed: 17420697]

Miao HQ, Hu K, et al. Potent neutralization of VEGF biological activities with a fully human antibody Fab fragment directed against VEGF receptor 2. Biochemical and Biophysical Research Communications. 2006; 345:438-445. [PubMed: 16682007]

Michaelson IC. Vascular morphogenesis in the retina of the cat. J Anat. 1948; 82:167-174.

Michaelson IC, Herz N, et al. Effect of increased oxygen on the development of the retinal vessels; an experimental study. Br J Ophthalmol. 1954; 38:577-587. [PubMed: 13199228]

Michels S, Rosenfeld PJ, et al. Systemic bevacizumab (Avastin) therapy for neovascular age-related macular degeneration: twelve-week results of an uncontrolled open-label clinical study. Ophthalmology. 2005; 112:1035-1047. [PubMed: 15936441]

Miller JW, Adamis AP, et al. Vascular endothelial growth factor in ocular neovascularization and proliferative diabetic retinopathy. Diabetes Metab Rev. 1997; 13:37-50. [PubMed: 9134347]

Misko TP, Highkin MK, et al. Characterization of the cytoprotective action of peroxynitrite decomposition catalysts. J Biol Chem. 1998; 273:15646-15653. [PubMed: 9624158]

Mitamura Y, Takeuchi S, et al. Monocyte chemotactic protein-1 in the vitreous of patients with proliferative diabetic retinopathy. Ophthalmologica. 2001; 215:415-418. [PubMed: 11741107]

Miyahara S, Kiryu J, et al. Simvastatin inhibits leukocyte accumulation and vascular permeability in the retinas of rats with streptozotocin-induced diabetes. Am J Pathol. 2004; 164:1697-1706.

[PubMed: 15111316] 
Miyamoto K, Khosrof S, et al. Prevention of leukostasis and vascular leakage in streptozotocininduced diabetic retinopathy via intercellular adhesion molecule-1 inhibition. PNAS. 1999; 96:10836-10841. [PubMed: 10485912]

Mizutani M, Kern TS, et al. Accelerated death of retinal microvascular cells in human and experimental diabetic retinopathy. J Clin Invest. 1996; 97:2883-2890. [PubMed: 8675702]

Morrison DG, Aschner M, et al. Vascular endothelial growth factor response to insulin-like growth factor in normoxic and hypoxic cell culture. Invest Ophthalmol Vis Sci. 2007; 48:E-Abstract 1735.

Mullins RF, Russell SR, et al. Drusen associated with aging and age-related macular degeneration contain proteins common to extracellular deposits associated with atherosclerosis, elastosis, amyloidosis, and dense deposit disease. FASEB J. 2000; 14:835-846. [PubMed: 10783137]

Murata T, Nakagawa K, et al. The relation between expression of vascular endothelial growth factor and breakdown of the blood-retinal barrier in diabetic rat retinas. Lab Invest. 1996; 74:819-825. [PubMed: 8606491]

Nakata K, Crabb JW, et al. Crystallin distribution in Bruch's membrane-choroid complex from AMD and age-matched donor eyes. Exp Eye Res. 2005; 80:821-826. [PubMed: 15939038]

Navaratna D, McGuire PG, et al. Proteolytic degradation of VE-cadherin alters the blood-retinal barrier in diabetes. Diabetes. 2007a; 56:2380-2387. [PubMed: 17536065]

Navaratna D, Menicucci G, et al. A peptide inhibitor of the uPA/uPAR system inhibits alteration of the blood-retinal barrier in diabetes. Invest Ophthalmol Vis Sci. 2007b; 48:E-Abstract 4025.

Neagoe PE, Lemieux C, et al. Vascular endothelial growth factor (VEGF)-A165-induced prostacyclin synthesis requires the activation of VEGF receptor-1 and -2 heterodimer. J Biol Chem. 2005; 280:9904-9912. [PubMed: 15637071]

Neu J, Afzal A, et al. The Dipeptide Arg-Gln Inhibits Retinal Neovascularization in the Mouse Model of Oxygen-Induced Retinopathy. Invest Ophthalmol Vis Sci. 2006; 47:3151-3155. [PubMed: 16799062]

Nguyen QD, Tatlipinar S, et al. Vascular endothelial growth factor is a critical stimulus for diabetic macular edema. Am J Ophthalmol. 2006; 142:961-969. [PubMed: 17046701]

Nilausen $\mathrm{K}$. The vasoformative tissue in the foetal retina with particular reference to the histochemical demonstration of its alkaline phosphatase activity. Acta Ophthalmol. 1958; 36:65-70. [PubMed: 13532616]

Nishijima K, Ng YS, et al. Vascular Endothelial Growth Factor-A Is a Survival Factor for Retinal Neurons and a Critical Neuroprotectant during the Adaptive Response to Ischemic Injury. Am J Pathol. 2007; 171:53-67. [PubMed: 17591953]

Nishimura M, Taniguchi Y. Retinal vascular patterns in the macula and the perimacular area in premature and full-term infants. Ophthalmologica. 1982; 185:147-157. [PubMed: 7133629]

Noda K, Ishida S, et al. Hypoxia induces the expression of membrane-type 1 matrix metalloproteinase in retinal glial cells. Invest Ophthalmol Vis Sci. 2005; 46:3817-3824. [PubMed: 16186369]

Notari L, Miller A, et al. Pigment Epithelium-Derived Factor Is a Substrate for Matrix Metalloproteinase Type 2 and Type 9: Implications for Downregulation in Hypoxia. Invest Ophthalmol Vis Sci. 2005; 46:2736-2747. [PubMed: 16043845]

Nozaki M, Raisler BJ, et al. Drusen complement components C3a and C5a promote choroidal neovascularization. Proc Natl Acad Sci U S A. 2006; 103:2328-2333. [PubMed: 16452172]

Obrosova IG, Minchenko AG, et al. Antioxidants attenuate early up regulation of retinal vascular endothelial growth factor in streptozotocin-diabetic rats. Diabetologia. 2001; 44:1102-1110. [PubMed: 11596663]

Obrosova IG, Pacher P, et al. Aldose reductase inhibition counteracts oxidative-nitrosative stress and poly(ADP-ribose) polymerase activation in tissue sites for diabetes complications. Diabetes. 2005; 54:234-242. [PubMed: 15616034]

Ogunshola OO, Antic A, et al. Paracrine and autocrine functions of neuronal vascular endothelial growth factor (VEGF) in the central nervous system. J Biol Chem. 2002; 277:11410-11415. [PubMed: 11777931] 
Oh H, Takagi H, et al. Selective induction of neuropilin-1 by vascular endothelial growth factor (VEGF): a mechanism contributing to VEGF-induced angiogenesis. Proc Natl Acad Sci USA. 2002; 99:383-388. [PubMed: 11756651]

Ohlsson A, Aher SM. Early erythropoietin for preventing red blood cell transfusion in preterm and/or low birth weight infants. Cochrane Database Syst Rev. 2006; 3:CD004863. [PubMed: 16856062]

Ohno-Matsui K, Hirose A, et al. Inducible expression of vascular endothelial growth factor in adult mice causes severe proliferative retinopathy and retinal detachment. Am J Path. 2002; 160:711719. [PubMed: 11839592]

Ohno-Matsui K, Morita I, et al. Novel mechanism for age-related macular degeneration: An equilibrium shift between the angiogenesis factors VEGF and PEDF. J Cell Physiol. 2001; 189:323-333. [PubMed: 11748590]

Okamoto N, Tobe T, et al. Transgenic mice with increased expression of vascular endothelial growth factor in the retina: a new model of intraretinal and subretinal neovascularization. Am J Pathol. 1997; 151:281-291. [PubMed: 9212753]

Okamoto T, Yamagishi S, et al. Angiogenesis induced by advanced glycation end products and its prevention by cerivastatin. Faseb J. 2002; 16:1928-1930. [PubMed: 12368225]

Oosthuyse B, Moons L, et al. Deletion of the hypoxia-response element in the vascular endothelial growth factor promoter causes motor neuron degeneration. Nat Genet. 2001; 28:131-138. [PubMed: 11381259]

Otani A, Kinder K, et al. Bone marrow-derived stem cells target retinal astrocytes and can promote or inhibit retinal angiogenesis. Nat Med. 2002; 8:1004-1010. [PubMed: 12145646]

Otrock ZK, Makarem JA, et al. Vascular endothelial growth factor family of ligands and receptors: review. Blood Cells Mol Dis. 2007; 38:258-268. [PubMed: 17344076]

Ozaki H, Yu AY, et al. Hypoxia inducible factor-1alpha is increased in ischemic retina: temporal and spatial correlation with VEGF expression. Invest Ophthalmol Vis Sci. 1999; 40:182-189. [PubMed: 9888442]

Ozawa K, Kondo T, et al. Expression of the oxygen-regulated protein ORP150 accelerates wound healing by modulating intracellular VEGF transport. J Clin Invest. 2001; 108:41-50. [PubMed: 11435456]

Palmer EA, Flynn JT, et al. Incidence and early course of retinopathy of prematurity. The Cryotherapy for Retinopathy of Prematurity Cooperative Group. Ophthalmology. 1991; 98:1628-1640. [PubMed: 1800923]

Patz A, Hoeck LE, et al. Studies on the effect of high oxygen administration in retrolental fibroplasia. I Nursery observations. Am J Ophthalmol. 1952; 35:1248-1253. [PubMed: 12976495]

Patz A. The role of oxygen in retrolental fibroplasia. Pediatrics. 1957; 19:504-524. [PubMed: 13408030]

Pe'er J, Shweiki D, et al. Hypoxia-induced expression of vascular endothelial growth factor by retinal cells is a common factor in neovascularizing ocular diseases. Lab Invest. 1995; 72:638-645. [PubMed: 7540233]

Penfold PL, Provis JM, et al. Angiogenesis in normal human retinal development: the involvement of astrocytes and macrophages. Graefes Arch Clin Exp Ophthalmol. 1990; 228:255-263. [PubMed: 2361598]

Penn JS, Henry MM, et al. Exposure to alternating hypoxia and hyperoxia causes severe proliferative retinopathy in the newborn rat. Pediatr Res. 1994; 36:724-731. [PubMed: 7898981]

Penn JS, Henry MM, et al. The range of $\mathrm{PaO} 2$ variation determines the severity of oxygen-induced retinopathy in newborn rats. Invest Ophthalmol Vis Sci. 1995; 36:2063-2070. [PubMed: 7657545]

Penn JS, Li TS, et al. Hypoxia reverses vascular attenuation in a transgenic mouse model of autosomal dominant retinitis pigmentosa. Invest Ophthalmol Vis Sci. 2000; 41:4007-4013. [PubMed: 11053306]

Penn JS, Tolman BL, et al. Variable oxygen exposure causes preretinal neovascularization in the newborn rat. Invest Ophthalmol Vis Sci. 1993; 34:576-585. [PubMed: 8449677] 
Perrin RM, Konopatskaya O, et al. Diabetic retinopathy is associated with a switch in splicing from anti- to pro-angiogenic isoforms of vascular endothelial growth factor. Diabetologia. 2005; 48:2422-2427. [PubMed: 16193288]

Pertovaara L, Kaipainen A, et al. Vascular endothelial growth factor is induced in response to transforming growth factor-beta in fibroblastic and epithelial cells. J Biol Chem. 1994; 269:6271-6274. [PubMed: 8119973]

Peterson LJ, Wittchen ES, et al. Heterotypic RPE-choroidal endothelial cell contact increases choroidal endothelial cell transmigration via PI 3-kinase and Rac1. Exp Eye Res. 2007 In Press.

Phelps DL. Reduced severity of oxygen-induced retinopathy in kittens recovered in $28 \%$ oxygen. Pediatr Res. 1988; 24:106-109. [PubMed: 3412844]

Phelps DL. Oxygen and Developmental Retinal Capillary Remodeling in the Kitten. Invest Ophthalmol Vis Sci. 1990; 31:2194-2200. [PubMed: 2211017]

Phelps DL, Rosenbaum AL. Effects of marginal hypoxemia on recovery from oxygen-induced retinopathy in the kitten model. Pediatrics. 1984; 73:1-6. [PubMed: 6546319]

Phelps DL, Rosenbaum AL. Effects of variable oxygenation and gradual withdrawal of oxygen during the recovery phase in oxygen-induced retinopathy: kitten model. Pediatr Res. 1987; 22:297-301. [PubMed: 3658549]

Pierce EA, Avery RL, et al. Vascular endothelial growth factor/vascular permeability factor expression in a mouse model of retinal neovascularization. Proc Natl Acad Sci U S A. 1995; 92:905-909. [PubMed: 7846076]

Pierce EA, Foley ED, et al. Regulation of vascular endothelial growth factor by oxygen in a model of retinopathy of prematurity. Arch Ophthalmol. 1996; 114:1219-1228. [PubMed: 8859081]

Platt DH, Bartoli M, et al. Peroxynitrite increases VEGF expression in vascular endothelial cells via STAT3. Free Radic Biol Med. 2005; 39:1353-1361. [PubMed: 16257644]

Poulaki V, Qin W, et al. Acute intensive insulin therapy exacerbates diabetic blood-retinal barrier breakdown via hypoxia-inducible factor-1alpha and VEGF. J Clin Invest. 2002; 109:805-15. [PubMed: 11901189]

Provis JM. Development of the primate retinal vasculature. Prog Retin Eye Res. 2001; 20:799-821. [PubMed: 11587918]

Provis JM, Leech J, et al. Development of the human retinal vasculature: cellular relations and VEGF expression. Exp Eye Res. 1997; 65:555-568. [PubMed: 9464188]

Provis JM, Sandercoe T. Astrocytes and blood vessels define the foveal rim during primate retinal development. Invest Ophthalmol Vis Sci. 2000; 41:2827-2836. [PubMed: 10967034]

Provis JM, van Driel D, et al. Development of the human retina: patterns of cell distribution and redistribution in the ganglion cell layer. J Comp Neurol. 1985; 233:429-451. [PubMed: 3980779]

Qaum T, Xu Q, et al. VEGF-initiated blood-retinal barrier breakdown in early diabetes. Invest Ophthalmol Vis Sci. 2001; 42:2408-2413. [PubMed: 11527957]

Rahimi N. Vascular endothelial growth factor receptors: Molecular mechanisms of activation and therapeutic potentials. Exp, Eye Res. 2006; 83:1005-1016. [PubMed: 16713597]

Rahimi N, Dayani V, et al. Receptor chimeras indicate that the vascular endothelial growth factor receptor-1 (VEGFR-1) modulates mitogenic activity of VEGFR-2 in endothelial cells. J Biol Chem. 2000; 275:16986-16992. [PubMed: 10747927]

Rakic JM, Lambert V, et al. Mice without uPA, tPA, or Plasminogen Genes Are Resistant to Experimental Choroidal Neovascularization. Invest Ophthalmol Vis Sci. 2003; 44:1732-1739. [PubMed: 12657615]

Rapisarda A, Uranchimeg B, et al. Identification of small molecule inhibitors of hypoxia-inducible factor 1 transcriptional activation pathway. Cancer Res. 2002; 62:4316-4324. [PubMed: 12154035]

Ratcliffe PJ. HIF-1 and HIF-2: working alone or together in hypoxia? J Clin Invest. 2007; 117:10681077. [PubMed: 17404621]

Raviola G. The structural basis of the blood-ocular barriers. Exp Eye Res. 1977; 25(Suppl):27-63. [PubMed: 412691] 
Rehman J, Li J, et al. Peripheral blood 'endothelial progenitor cells' are derived from monocyte/ macrophages and secrete angiogenic growth factors. Circulation. 2003; 107:1164-1169. [PubMed: 12615796]

Resnikoff S, Pascolini D, et al. Global data on visual impairment in the year 2002. Bull World Health Org. 2004; 82:844-851. [PubMed: 15640920]

Revised Indications for the Treatment of Retinopathy of Prematurity: Results of the Early Treatment for Retinopathy of Prematurity Randomized Trial. Early Treatment for Retinopathy of Prematurity Cooperative Group. Arch Ophthalmol. 2003; 121:1684-1694. [PubMed: 14662586]

Risau W. Mechanisms of angiogenesis. Nature. 1997; 386:671-674. [PubMed: 9109485]

Risau W, Flamme I. Vasculogenesis. Annu Rev Cell Dev Biol. 1995; 11:73-91. [PubMed: 8689573]

Rivera A, Fisher SA, et al. Hypothetical LOC387715 is a second major susceptibility gene for agerelated macular degeneration, contributing independently of complement factor $\mathrm{H}$ to disease risk. Hum Mol Genet. 2005; 14:3227-3236. [PubMed: 16174643]

Robbins RA, Nelson KJ, et al. Complement activation by cigarette smoke. Am J Physiol. 1991; 260:L254-L259. [PubMed: 1902064]

Robbins SG, Conaway JR, et al. Detection of vascular endothelial growth factor (VEGF) protein in vascular and non-vascular cells of the normal and oxygen-injured rat retina. Growth Factors. 1997; 14:279-295. [PubMed: 9386992]

Robbins SG, Rajaratnam VS, et al. Evidence for upregulation and redistribution of vascular endothelial growth factor (VEGF) receptors flt-1 and flk-1 in the oxygen-injured rat retina. Growth Factors. 1998; 16:1-9. [PubMed: 9777366]

Roberts WG, Palade GE. Increased microvascular permeability and endothelial fenestration induced by vascular endothelial growth factor. J Cell Sci. 1995; 108:2369-2379. [PubMed: 7673356]

Robinow S, Campos KM, et al. The elav gene product of Drosophila, required in neurons, has three RNP consensus motifs. Science. 1988; 242:1570-1572. [PubMed: 3144044]

Robinson GS, Ju M, et al. Nonvascular role for VEGF: VEGFR-1, 2 activity is critical for neural retinal development. Faseb J. 2001; 15:1215-1217. [PubMed: 11344092]

Romagnoli C, Zecca E, et al. Do recombinant human erythropoietin and iron supplementation increase the risk of retinopathy of prematurity? Eur J Pediatr. 2000; 159:627-628. [PubMed: 10968244]

Romero MJ, Platt DH, et al. Does Elevated Arginase Activity Contribute to Diabetes-Induced Endothelial Dysfunction. Circulation. submitted.

Rosenfeld PJ, Moshfeghi AA, et al. Optical coherence tomography findings after an intravitreal injection of bevacizumab (avastin) for neovascular age-related macular degeneration. Ophthalmic Surg Lasers Imaging. 2005; 36:331-335. [PubMed: 16156152]

Rosenfeld PJ, Brown DM, et al. Ranibizumab for Neovascular Age-Related Macular Degeneration. N Engl J Med. 2006a; 355:1419-1431. [PubMed: 17021318]

Rosenfeld PJ, Heier JS, et al. Tolerability and efficacy of multiple escalating doses of ranibizumab (Lucentis) for neovascular age-related macular degeneration. Ophthalmology. 2006b; 113:632.

Rousseau S, Houle F, et al. p38 MAP kinase activation by vascular endothelial growth factor mediates actin reorganization and cell migration in human endothelial cells. Oncogene. 1997; 15:21692177. [PubMed: 9393975]

Rudolf M, Winkler B, et al. Increased expression of vascular endothelial growth factor associated with accumulation of lipids in Bruch's membrane of LDL receptor knockout mice. Br J Ophthalmol. 2005; 89:1627-1630. [PubMed: 16299144]

Ryuto M, Ono M, et al. Induction of vascular endothelial growth factor by tumor necrosis factor alpha in human glioma cells. Possible roles of SP-1. J Biol Chem. 1996; 271:28220-28228. [PubMed: 8910439]

Saint-Geniez M, Maldonado AE, et al. VEGF Expression and Receptor Activation in the Choroid during Development and in the Adult. Invest Ophthalmol Vis Sci. 2006; 47:3135-3142. [PubMed: 16799060]

Saint-Geniez M, D'Amore PA. Development and pathology of the hyaloid, choroidal and retinal vasculature. Int J Dev Biol. 2004; 48:1045-1058. [PubMed: 15558494] 
Saishin Y, Saishin Y, Takahashi K, et al. VEGF-TRAP(R1R2) suppresses choroidal neovascularization and VEGF-induced breakdown of the blood-retinal barrier. J Cell Physiol. 2003; 195:241-248. [PubMed: 12652651]

Sakai R, Henderson JT, et al. The mammalian ShcB and ShcC phosphotyrosine docking proteins function in the maturation of sensory and sympathetic neurons. Neuron. 2000; 28:819-833. [PubMed: 11163269]

Sakamoto T, Sakamoto H, et al. Vessel formation by choroidal endothelial cells in vitro is modulated by retinal pigment epithelial cells. Arch Ophthalmol. 1995; 113:512-520. [PubMed: 7536000]

Sakurai Y, Ohgimoto K, et al. Essential role of Flk-1 (VEGF receptor 2) tyrosine residue 1173 in vasculogenesis in mice. Proc Natl Acad Sci. 2005; 102:1076-1081. [PubMed: 15644447]

Salhia B, Angelov L, et al. Expression of vascular endothelial growth factor by reactive astrocytes and associated neoangiogenesis. Brain Res. 2000; 883:87-97. [PubMed: 11063991]

Salvemini D, Wang ZQ, et al. Peroxynitrite decomposition catalysts: therapeutics for peroxynitritemediated pathology. Proc Natl Acad Sci U S A. 1998; 95:2659-2663. [PubMed: 9482943]

Sandercoe TM, Madigan MC, et al. Astrocyte proliferation during development of the human retinal vasculature. Exp Eye Res. 1999; 69:511-523. [PubMed: 10548471]

Sato TN, Tozawa Y. Distinct roles of the receptor tyrosine kinases Tie-1 and Tie-2 in blood vessel formation. Nature. 1995; 376:70-74. [PubMed: 7596437]

Scalia R, Stalker TJ. Microcirculation as a target for the anti-inflammatory properties of statins. Microcirculation. 2002; 9:431-42. [PubMed: 12483541]

Schaefer LK, Ren Z, et al. Constitutive activation of Stat3alpha in brain tumors: localization to tumor endothelial cells and activation by the endothelial tyrosine kinase receptor (VEGFR-2). Oncogene. 2002; 21:2058-2065. [PubMed: 11960378]

Schafer M, Schafer C, et al. Role of redox signaling in the autonomous proliferative response of endothelial cells to hypoxia. Circ Res. 2003; 92:1010-1015. [PubMed: 12690038]

Schatteman GC, Hanlon HD, et al. Blood-derived angioblasts accelerate blood-flow restoration in diabetic mice. J Clin Invest. 2000; 106:571-578. [PubMed: 10953032]

Schofield CJ, Ratcliffe PJ. Oxygen sensing by HIF hydroxylases. Nat Rev Mol Cell Biol. 2004; 5:343354. [PubMed: 15122348]

Schwesinger C, Yee C, et al. Intrachoroidal neovascularization in transgenic mice overexpressing vascular endothelial growth factor in the retinal pigment epithelium. Am J Path. 2001; 158:11611172. [PubMed: 11238064]

Seddon JM, Gensler G, et al. Association between C-reactive protein and age-related macular degeneration. JAMA. 2004; 291:704-710. [PubMed: 14871913]

Seddon JM, George S, et al. Cigarette smoking, fish consumption, omega-3 fatty acid intake, and associations with age-related macular degeneration: the US Twin Study of Age-Related Macular Degeneration. Arch Ophthalmol. 2006a; 124:995-1001. [PubMed: 16832023]

Seddon JM, George S, Rosner, et al. CFH Gene Variant, Y402H, and Smoking, Body Mass Index, Environmental Associations with Advanced Age-Related Macular Degeneration. Hum Hered. 2006b; 61:157-165. [PubMed: 16816528]

Seddon JM, Willett WC, et al. A prospective study of cigarette smoking and age-related macular degeneration in women. JAMA. 1996; 276:1141-1146. [PubMed: 8827966]

Senger DR, Galli SJ, et al. Tumor cells secrete a vascular permeability factor that promotes accumulation of ascites fluid. Science. 1983; 219:983-985. [PubMed: 6823562]

Shankar A, Mitchell P, et al. Association between Circulating White Blood Cell Count and Long-Term Incidence of Age-related Macular Degeneration: The Blue Mountains Eye Study. Am J Epidemiol. 2007; 165:375-382. [PubMed: 17110636]

Sheetz MJ, King GL. Molecular understanding of hyperglycemia's adverse effects for diabetic complications. Jama. 2002; 288:2579-2588. [PubMed: 12444865]

Sheraidah G, Steinmetz R, Maguire, et al. Correlation between lipids extracted from Bruch's membrane and age. Ophthalmology. 1993; 100:47-51. [PubMed: 8433826] 
Shi Q, Le X, et al. Constitutive Sp1 activity is essential for differential constitutive expression of vascular endothelial growth factor in human pancreatic adenocarcinoma. Cancer Res. 2001; 61:4143-4154. [PubMed: 11358838]

Shibuya M, Ito N, et al. Structure and function of vascular endothelial growth factor receptor-1 and -2 . Curr Top Microbiol Immunol. 1999; 237:59-83. [PubMed: 9893346]

Shih SC, Ju M, et al. Selective stimulation of VEGFR-1 prevents oxygen-induced retinal vascular degeneration in retinopathy of prematurity. J Clin Invest. 2003; 112:50-57. [PubMed: 12840058]

Shima DT, Kuroki M, et al. The mouse gene for vascular endothelial growth factor. Genomic structure, definition of the transcriptional unit, and characterization of transcriptional and posttranscriptional regulatory sequences. J Biol Chem. 1996; 271:3877-3883. [PubMed: 8632007]

Sho K, Takahashi K, et al. Polypoidal Choroidal Vasculopathy: Incidence, Demographic Features, and Clinical Characteristics. Arch Ophthalmol. 2003; 121:1392-1396. [PubMed: 14557174]

Shweiki D, Itin A, et al. Vascular endothelial growth factor induced by hypoxia may mediate hypoxiainitiated angiogenesis. Nature. 1992; 359:843-845. [PubMed: 1279431]

Simpson DA, Murphy GM, et al. Expression of the VEGF gene family during retinal vaso-obliteration and hypoxia. Biochem Biophys Res Commun. 1999; 262:333-340. [PubMed: 10462475]

Sjolie AK, Stephenson J, et al. Retinopathy and vision loss in insulin-dependent diabetes in Europe. The EURODIAB IDDM Complications Study. Ophthalmology. 1997; 104:252-260. [PubMed: 9052629]

Smith G, McLeod D, et al. Immunolocalisation of the VEGF receptors FLT-1, KDR, and FLT-4 in diabetic retinopathy. Br J Ophthalmol. 1999; 83:486-494. [PubMed: 10434875]

Smith LE, Wesolowski E, et al. Oxygen-induced retinopathy in the mouse. Invest Ophthalmol Vis Sci. 1994; 35:101-111. [PubMed: 7507904]

Smith LE, Shen W, et al. Regulation of vascular endothelial growth factor-dependent retinal neovascularization by insulin-like growth factor-1 receptor. Nat Med. 1999; 5:1390-1395. [PubMed: 10581081]

Soker S, Miao HQ, et al. VEGF165 mediates formation of complexes containing VEGFR-2 and neuropilin-1 that enhance VEGF165-receptor binding. J Cell Biochem. 2002; 85:357-368. [PubMed: 11948691]

Sonta T, Inoguchi T, et al. Evidence for contribution of vascular NAD(P)H oxidase to increased oxidative stress in animal models of diabetes and obesity. Free Radic Biol Med. 2004; 37:115123. [PubMed: 15183199]

Soriano FG, Pacher P, et al. Rapid reversal of the diabetic endothelial dysfunction by pharmacological inhibition of poly(ADP-ribose) polymerase. Circ Res. 2001; 89:684-691. [PubMed: 11597991]

Sowers JR. Effects of statins on the vasculature: Implications for aggressive lipid management in the cardiovascular metabolic syndrome. Am J Cardiol. 2003; 91:14B-22B.

Spaide RF, Armstrong D, et al. Continuing medical education review: choroidal neovascularization in age-related macular degeneration--what is the cause? Retina. 2003; 23:595-614. [PubMed: 14574243]

Sreekumar PG, Kannan R, et al. Thiol regulation of vascular endothelial growth factor-A and its receptors in human retinal pigment epithelial cells. Biochem Biophys Res Commun. 2006; 346:1200-1206. [PubMed: 16793007]

Stalmans I, Ng YS, et al. Arteriolar and venular patterning in retinas of mice selectively expressing VEGF isoforms. J Clin Invest. 2002; 109:327-336. [PubMed: 11827992]

Starita C, Patel M, et al. Vascular endothelial growth factor and the potential therapeutic use of pegaptanib (macugen) in diabetic retinopathy. Dev Ophthalmol. 2007; 39:122-148. [PubMed: 17245083]

Stevens TS, Bressler NM, et al. Occult choroidal neovascularization in age-related macular degeneration. A natural history study. Arch Ophthalmol. 1997; 115:345-350. [PubMed: 9076206]

Stitt AW, Simpson DA, et al. Expression of vascular endothelial growth factor (VEGF) and its receptors is regulated in eyes with intra-ocular tumours. J Pathol. 1998; 186:306-312. [PubMed: 10211121] 
Stone EM, Braun TA, et al. Missense variations in the fibulin 5 gene and age-related macular degeneration. N Engl J Med. 2004; 351:346-353. [PubMed: 15269314]

Stone J, Itin A, et al. Development of retinal vasculature is mediated by hypoxia-induced vascular endothelial growth factor (VEGF) expression by neuroglia. J Neurosci. 1995; 15:4738-4747. [PubMed: 7623107]

Stone J, Chan-Ling T, et al. Roles of vascular endothelial growth factor and astrocyte degeneration in the genesis of retinopathy of prematurity. Invest Ophthalmol Vis Sci. 1996; 37:290-299. [PubMed: 8603833]

Stone J, Dreher Z. Relationship between astrocytes, ganglion cells and vasculature of the retina. J Comp Neurol. 1987; 255:35-49. [PubMed: 3819008]

STOP-ROP Multicenter Study Group. Supplemental Therapeutic Oxygen for Prethreshold Retinopathy Of Prematurity (STOP-ROP), a randomized, controlled trial. I: primary outcomes Pediatrics. 2000; 105:295-310.

Storkebaum E, Lambrechts D, Dewerchin M, et al. Treatment of motoneuron degeneration by intracerebroventricular delivery of VEGF in a rat model of ALS. Nat Neurosci. 2005; 8:85-92. [PubMed: 15568021]

Sun FY, Guo X. Molecular and cellular mechanisms of neuroprotection by vascular endothelial growth factor. J Neurosci Res. 2007; 79:180-184. [PubMed: 15573409]

Sunness JS, Gonzalez-Baron J, et al. The development of choroidal neovascularization in eyes with the geographic atrophy form of age-related macular degeneration. Ophthalmology. 1999; 106:910919. [PubMed: 10328389]

Suri C, Jones PF, et al. Requisite role of angiopoietin-1, a ligand for the TIE2 receptor, during embryonic angiogenesis. Cell. 1996; 87:1171-1180. [PubMed: 8980224]

Takagi H, King GL, et al. Identification and characterization of vascular endothelial growth factor receptor (Flt) in bovine retinal pericytes. Diabetes. 1996; 45:1016-1023. [PubMed: 8690146]

Takagi H, Koyama S, et al. Potential role of the angiopoietin/tie2 system in ischemia-induced retinal neovascularization. Invest Opthalmol Vis Sci. 2003; 44:393-402.

Takahashi H, Obata R, et al. A novel vascular endothelial growth factor receptor 2 inhibitor, SU11248, suppresses choroidal neovascularization in vivo. J Ocul Pharmacol Ther. 2006; 22:213-218. [PubMed: 16910860]

Takahashi H, Yanagi Y, et al. Contribution of bone-marrow-derived cells to choroidal neovascularization. Biochemical and Biophysical Research Communications. 2004; 320:372375. [PubMed: 15219837]

Takahashi H, Shibuya M. The vascular endothelial growth factor (VEGF)/VEGF receptor system and its role under physiological and pathological conditions. Clin Sci (Lond). 2005; 109:227-241. [PubMed: 16104843]

Takahashi K, Saishin Y, et al. Intraocular expression of endostatin reduces VEGF-induced retinal vascular permeability, neovascularization, and retinal detachment. Faseb J. 2003; 28:28.

Takahashi T, Yamaguchi S, et al. A single autophosphorylation site on KDR/Flk-1 is essential for VEGF-A-dependent activation of PLC- $\gamma$ and DNA synthesis in vascular endothelial cells. EMBO J. 2001; 20:2768-2678. [PubMed: 11387210]

Takeda M, Mori F, et al. Constitutive nitric oxide synthase is associated with retinal vascular permeability in early diabetic rats. Diabetologia. 2001; 44:1043-1050. [PubMed: 11484083]

Taomoto M, McLeod DS, et al. Localization of adenosine A2a receptor in retinal development and oxygen-induced retinopathy. Invest Ophthalmol Vis Sci. 2000; 41:230-243. [PubMed: 10634625]

Tatar O, Adam A, et al. Expression of VEGF and PEDF in Choroidal Neovascular Membranes Following Verteporfin Photodynamic Therapy. Am J Ophthalmol. 2006; 142:95. [PubMed: 16815256]

Tepper OM, Galiano RD, et al. Human endothelial progenitor cells from type II diabetics exhibit impaired proliferation, adhesion, and incorporation into vascular structures. Circulation. 2002; 106:2781-2786. [PubMed: 12451003] 
Thelemann A, Petti F, et al. Phosphotyrosine signaling networks in epidermal growth factor receptor overexpressing squamous carcinoma cells. Mol Cell Proteomics. 2005; 4:356-76. [PubMed: 15657067]

Tian J, Ishibashi K, et al. Advanced glycation endproduct-induced aging of the retinal pigment epithelium and choroid: A comprehensive transcriptional response. PNAS. 2005; 102:1184611851. [PubMed: 16081535]

Tin W, Milligan DW, et al. Pulse oximetry, severe retinopathy, and outcome at one year in babies of less than 28 weeks gestation. Arch Dis Child Fetal Neonatal Ed. 2001; 84:F106-110. [PubMed: 11207226]

Treins C, Giorgetti-Peraldi S, et al. Insulin stimulates hypoxia-inducible factor 1 through a phosphatidylinositol 3-kinase/target of rapamycin-dependent signaling pathway. J Biol Chem. 2002; 277:27975-27981. [PubMed: 12032158]

UKPDS. Effect of intensive blood-glucose control with metformin on complications in overweight patients with type 2 diabetes (UKPDS 34). Lancet. 1998; 352:854-865. [PubMed: 9742977]

Urbich S, Dimmeler S. Endothelial progenitor cells characterization and role in vascular biology. Circ Res. 2004; 95:343-353. [PubMed: 15321944]

Ushio-Fukai M, Tang Y, et al. Novel role of gp91(phox)-containing NAD(P)H oxidase in vascular endothelial growth factor-induced signaling and angiogenesis. Circ Res. 2002; 91:1160-1167. [PubMed: 12480817]

Usui T, Ishida S, et al. VEGF164(165) as the pathological isoform: differential leukocyte and endothelial responses through VEGFR1 and VEGFR2. Invest Ophthalmol Vis Sci. 2004; 45:368-374. [PubMed: 14744874]

van der Velden AW, Thomas AA. The role of the $5^{\prime}$ untranslated region of an mRNA in translation regulation during development. Int J Biochem Cell Biol. 1999; 31:87-106. [PubMed: 10216946]

Vinores SA, Derevjanik NL, et al. Vascular endothelial growth factor (VEGF), transforming growth factor-beta (TGFbeta), and interleukin-6 (IL-6) in experimental herpesvirus retinopathy: association with inflammation and viral infection. Histol Histopathol. 2001; 16:1061-1071. [PubMed: 11642726]

Vuorela P, Helske S, et al. Amniotic Fluid-Soluble Vascular Endothelial Growth Factor Receptor-1 in Preeclampsia. Obstet Gynecol. 2000; 95:353-357. [PubMed: 10711543]

Wang F, Rendahl KG, et al. AAV-Mediated Expression of Vascular Endothelial Growth Factor Induces Choroidal Neovascularization in Rat. Invest Ophthalmol Vis Sci. 2003; 44:781-790. [PubMed: 12556414]

Wang GL, Semenza GL. Purification and characterization of hypoxia-inducible factor 1. J Biol Chem. 1995; 270:1230-1237. [PubMed: 7836384]

Watanabe T, Raff MC. Retinal astrocytes are immigrants from the optic nerve. Nature. 1988; 332:834837. [PubMed: 3282180]

Watanabe D, Suzuma K, et al. Erythropoietin as a retinal angiogenic factor in proliferative diabetic retinopathy. N Engl J Med. 2005; 353:782-792. [PubMed: 16120858]

Weber BHF, Vogt G, et al. Mutations in the tissue inhibitor of metalloproteinases-3 (TIMP3) in patients with Sorsby's fundus dystrophy. Nat Genet. 1994; 8:352-356. [PubMed: 7894485]

Weis M, Heeschen C, et al. Statins have biphasic effects on angiogenesis. Circulation. 2002; 105:739_ 745. [PubMed: 11839631]

Wenger RH. Cellular adaptation to hypoxia: O2-sensing protein hydroxylases, hypoxia-inducible transcription factors, and O2-regulated gene expression. FASEB J. 2002; 16:1151-62. [PubMed: 12153983]

Werdich XQ, McCollum GW, et al. Variable oxygen and retinal VEGF levels: correlation with incidence and severity of pathology in a rat model of oxygen-induced retinopathy. Exp Eye Res. 2004; 79:623-30. [PubMed: 15500821]

West H, Richardson WD, et al. Stabilization of the retinal vascular network by reciprocal feedback between blood vessels and astrocytes. Development. 2005; 132:1855-1862. [PubMed: 15790963] 
Wilkinson-Berka JL, Babic S, et al. Inhibition of platelet-derived growth factor promotes pericyte loss and angiogenesis in ischemic retinopathy. Am J Pathol. 2004; 164:1263-1273. [PubMed: 15039215]

Witmer AN, Vrensen GFJM, et al. Vascular endothelial growth factors and angiogenesis in eye disease. Prog Retin Eye Res. 2003; 22:1-29. [PubMed: 12597922]

Xu Q, Briggs J, et al. Targeting Stat3 blocks both HIF-1 and VEGF expression induced by multiple oncogenic growth signaling pathways. Oncogene. 2005; 24:5552-5560. [PubMed: 16007214]

Yamada Y, Ishibashi K, et al. The expression of advanced glycation endproduct receptors in rpe cells associated with basal deposits in human maculas. Exp Eye Res. 2006; 82:840-848. [PubMed: 16364296]

Yamagishi S, Yonekura H, et al. Advanced glycation end products-driven angiogenesis in vitro. Induction of the growth and tube formation of human microvascular endothelial cells through autocrine vascular endothelial growth factor. J Biol Chem. 1997; 272:8723-8730. [PubMed: 9079706]

Yamagishi S, Nakamura K, et al. Pigment epithelium-derived factor inhibits advanced glycation end product-induced retinal vascular hyperpermeability by blocking reactive oxygen speciesmediated vascular endothelial growth factor expression. J Biol Chem. 2006; 281:20213-20220. [PubMed: 16707486]

Yancopoulos GD, Davis S, et al. Vascular-specific growth factors and blood vessel formation. Nature. 2000; 407:242-248. [PubMed: 11001067]

Yang JC, Haworth L, et al. A Randomized Trial of Bevacizumab, an Anti-Vascular Endothelial Growth Factor Antibody, for Metastatic Renal Cancer. N Engl J Med. 2003; 349:427-434. [PubMed: 12890841]

Yang J, Duh E, et al. PEDF's Action Inhibiting VEGF-Induced Permeability in Retinal Endothelial Cells Involves Beta-Catenin Signaling Pathway and uPAR Gene Expression. Invest Ophthalmol Vis Sci. 2007; 48:E-Abstract 1777.

Yang K, Cepko CL. Flk-1, a receptor for vascular endothelial growth factor (VEGF), is expressed by retinal progenitor cells. J Neurosci. 1996; 16:6089-6099. [PubMed: 8815891]

Yannuzzi LA, Negrao S, et al. Retinal angiomatous proliferation in age-related macular degeneration. Retina. 2001; 21:416-434. [PubMed: 11642370]

Yi X, Ogata N, et al. Vascular endothelial growth factor expression in choroidal neovascularization in rats. Graefe's Arch Clin Exp Ophthalmol. 1997; 235:313-319. [PubMed: 9176680]

Yi X, Mai LC, et al. Time-course expression of vascular endothelial growth factor as related to the development of the retinochoroidal vasculature in rats. Exp Brain Res. 1998; 118:155-160. [PubMed: 9547084]

Yoo PS, Mulkeen AL, et al. Post-transcriptional regulation of vascular endothelial growth factor: implications for tumor angiogenesis. World J Gastroenterol. 2006; 12:4937-4942. [PubMed: 16937487]

York JR, Landers S, et al. Arterial oxygen fluctuation and retinopathy of prematurity in very-lowbirth-weight infants. J Perinatol. 2004; 24:82-87. [PubMed: 14762452]

Young TL, Anthony DC, et al. Histopathology and vascular endothelial growth factor in untreated and diode laser-treated retinopathy of prematurity. J AAPOS. 1997; 1:105-110. [PubMed: 10875087]

Yu DY, Cringle SJ. Oxygen distribution and consumption within the retina in vascularised and avascular retinas and in animal models of retinal disease. Prog Retin Eye Res. 2001; 20:175-208. [PubMed: 11173251]

Zeng H, Sanyal S, et al. Tyrosine residues 951 and 1059 of vascular endothelial growth factor receptor-2 (KDR) are essential for vascular permeability factor/vascular endothelial growth factor induced endothelium migration and proliferation, respectively. J Biol Chem. 2001; 276:32714-32719. [PubMed: 11435426]

Zhang SX, Wang JJ, et al. Pigment epithelium-derived factor downregulates vascular endothelial growth factor (VEGF) expression and inhibits VEGF-VEGF receptor 2 binding in diabetic retinopathy. J Mol Endocrinol. 2006b; 37:1-12. [PubMed: 16901919]

Zhang SX, Wang JJ, et al. Pigment epithelium-derived factor (PEDF) is an endogenous antiinflammatory factor. Faseb J. 2006a; 20:323-325. [PubMed: 16368716] 
Zhang Y, Stone J. Role of astrocytes in the control of developing retinal vessels. Invest Ophthalmol Vis Sci. 1997; 38:1653-1666. [PubMed: 9286254]

Zhong H, Chiles K, et al. Modulation of hypoxia-inducible factor 1alpha expression by the epidermal growth factor/phosphatidylinositol 3-kinase/PTEN/AKT/FRAP pathway in human prostate cancer cells: implications for tumor angiogenesis and therapeutics. Cancer Res. 2000; 60:15411545. [PubMed: 10749120]

Zhou J, Jang YP, et al. Complement activation by photooxidation products of A2E, a lipofuscin constituent of the retinal pigment epithelium. PNAS. 2006; 103:16182-16187. [PubMed: 17060630]

Zubilewicz A, Hecquet C, et al. Two distinct signalling pathways are involved in FGF2-stimulated proliferation of choriocapillary endothelial cells: A comparative study with VEGF. Oncogene. 2001; 20:1403-1413. [PubMed: 11313884] 


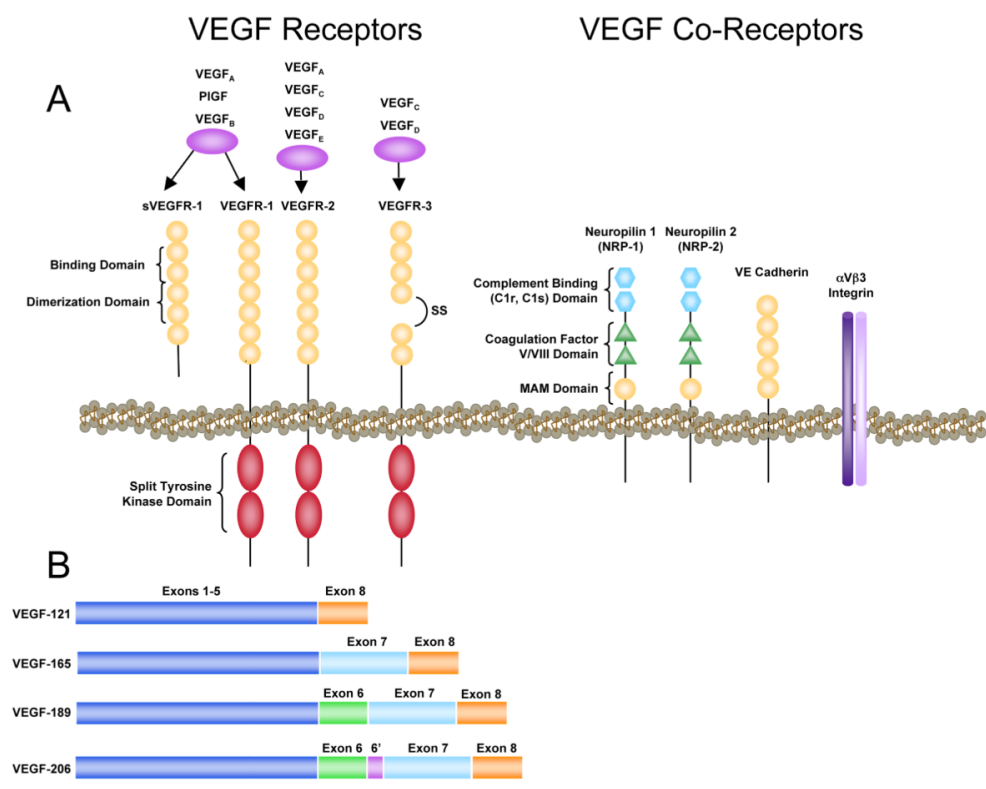

Figure 1.

A) Associations of various VEGF isoforms with VEGF receptors and co-receptors and basic receptor structure. B) Variants of VEGF-A are formed by alternative splicing. Exons 1 - 5 span the receptor binding domain, while exons 6 and 7 span the heparin binding domain. 


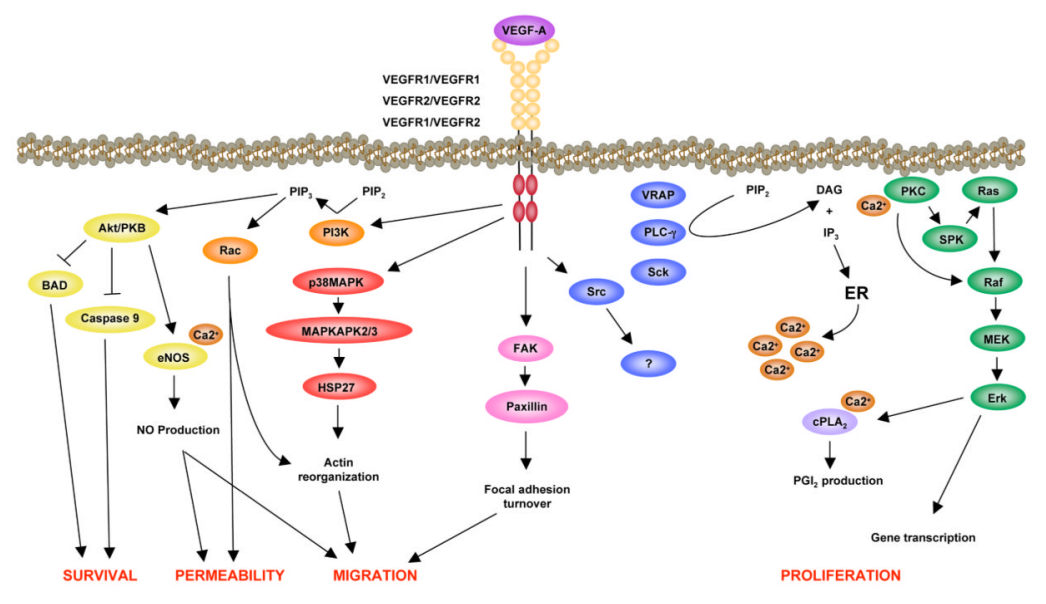

Figure 2.

VEGFR-2 signaling pathways leading to endothelial cell activities designated in red. 


\section{Adequate oxygenation: HIF inactive}

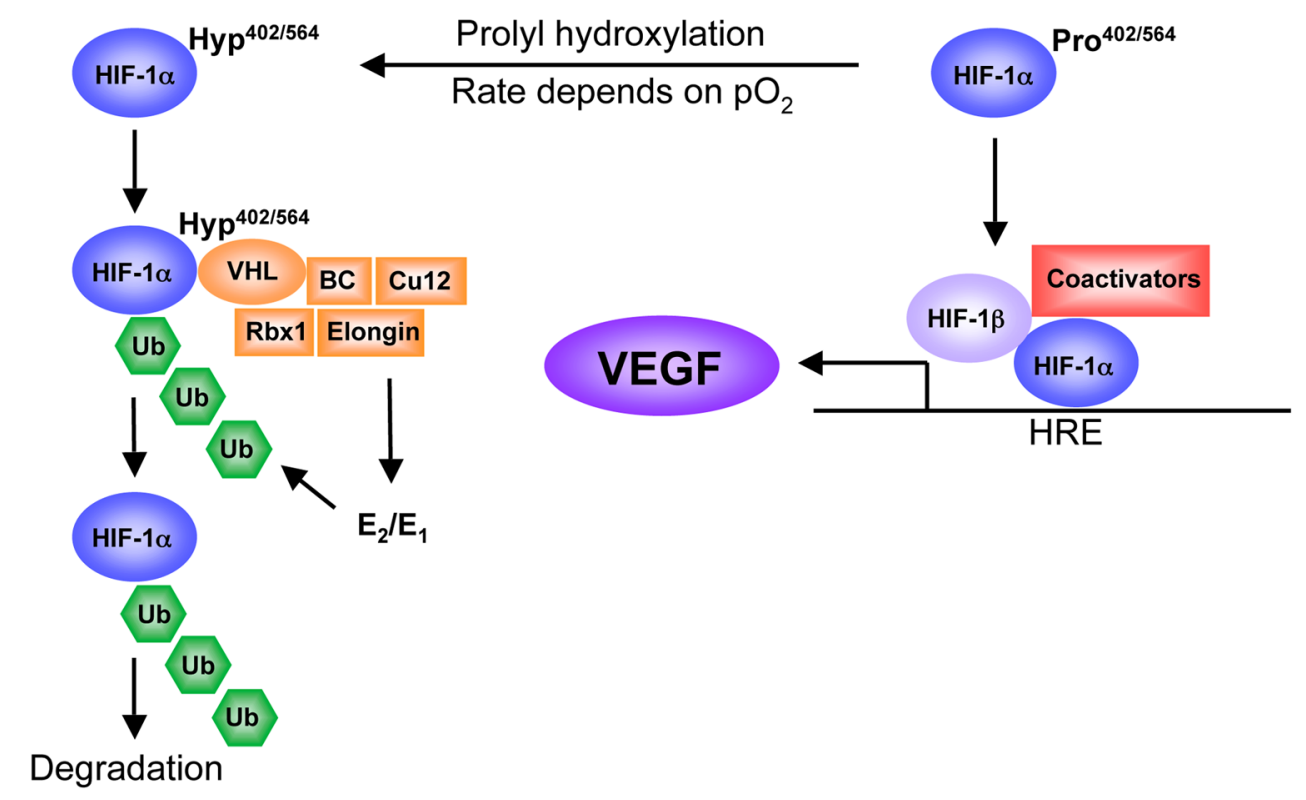

Hypoxia: HIF active
Figure 3.

In normoxia, $\mathrm{pVHL}$ binds a hydroxylated proline residue of HIF-1a, leading to ubiquitin attachment and degradation in the proteasome. In hypoxia, constitutively expressed HIF-1a dimerizes with HIF- $1 \beta$ and leads to transcription of hypoxia-inducible genes, including VEGF. 


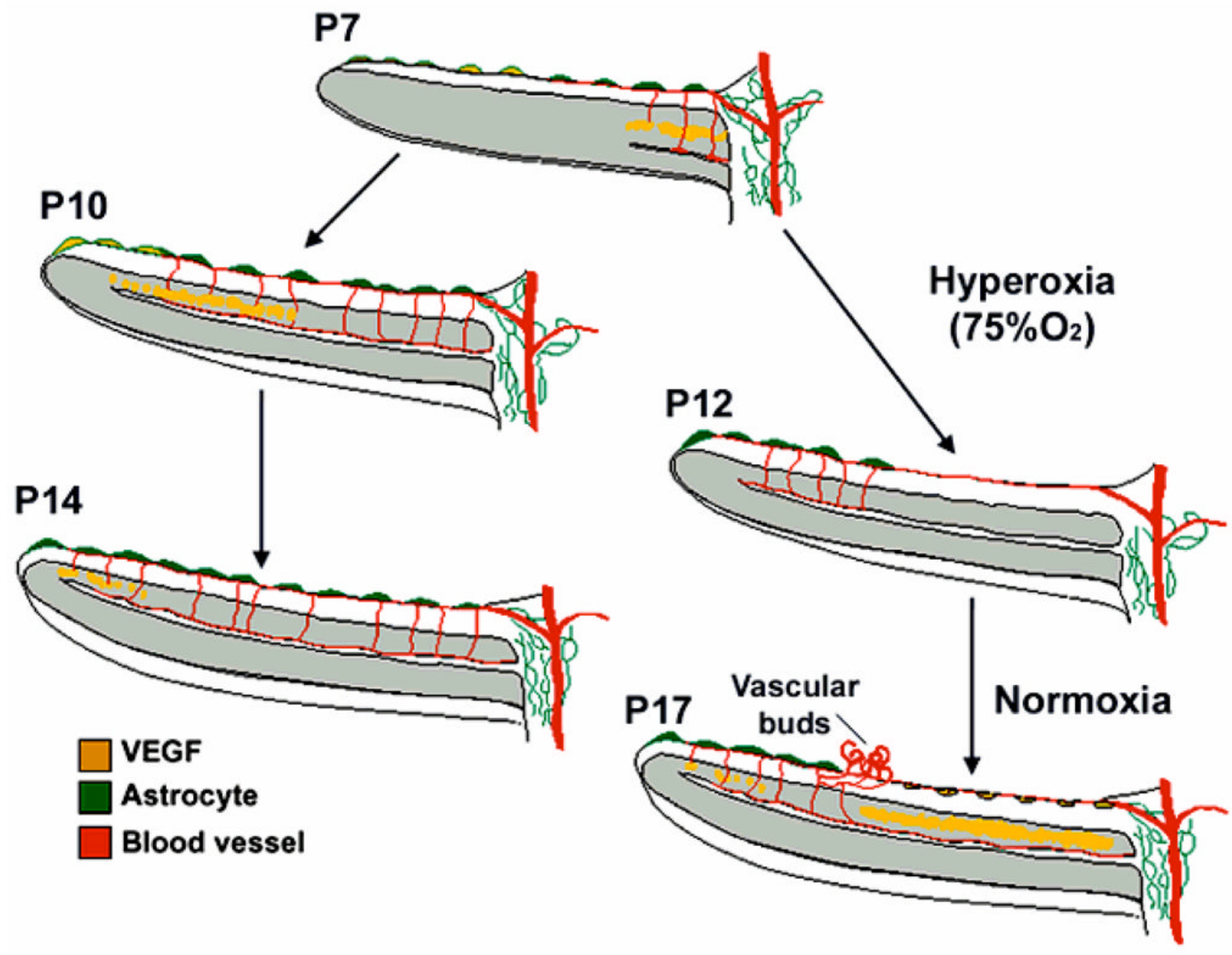

Figure 4.

Mouse model of retinal vascularization and OIR showing the temporal and spatial relationships between astrocyte growth, VEGF production and blood vessel development (Republished with permission of the University of the Basque Country Press from SaintGeniez M, D'Amore PA. Development and pathology of the hyaloid, choroidal and retinal vasculature. Int J Dev Biol 2004; 48:1045-58). 

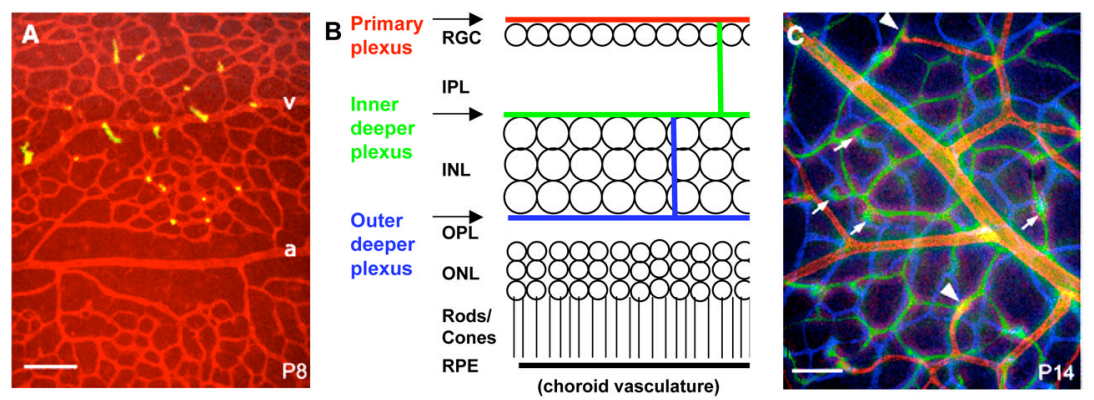

Figure 5.

Vascular networks in the retina are depicted by fluorescein-labeled lectin staining in retinal whole mount preparations (A, C) and in a schematic of a retinal cross-section (B). Images in $\mathrm{A}$ and $\mathrm{C}$ were taken at different focal planes and colored red (primary plexus), green (inner deeper plexus) and blue (outer deeper plexus), and superimposed using a computer. At P8 (A) sprouts (yellow) are emerging from veins (v) and capillaries but not arteries (a). At P14 (C) all three networks are established. Arrowheads indicate connections between the primary and the inner deeper plexus. Arrows indicate connections between the inner and outer deeper plexus (RGC, retinal ganglion cells; IPL, inner plexiform layer; INL, inner nuclear layer; OPL, outer plexiform layer; ONL, outer nuclear layer; RPE, retinal pigment epithelium). Scale bars are $100 \mu \mathrm{m}$. Adapted from Fruttiger, 2007. 

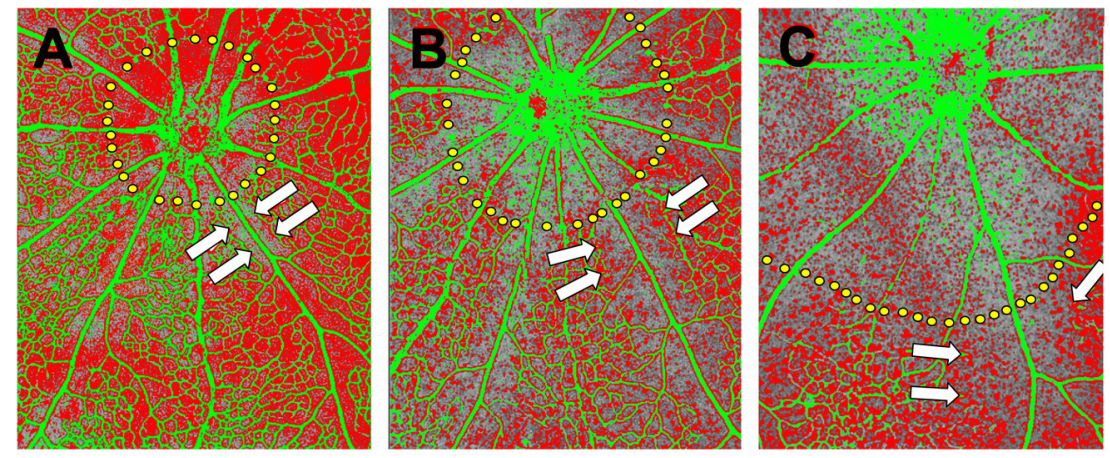

Figure 6.

P6 mice were exposed to $20 \%$ (A), $40 \%$ (B) or $80 \%$ (C) oxygen for 36 hours. Retinas were subsequently processed for in situ hybridization with a probe against mouse VEGF mRNA which is pseudo-colored red, while vessels were visualized with an antibody against mouse collagen type IV which is pseudo-colored green. Capillary-free zones around the optic nerve head (dotted yellow lines) and around retinal arteries (arrows) expand with increasing oxygen concentrations. Adapted from Claxton and Fruttiger, 2003. 

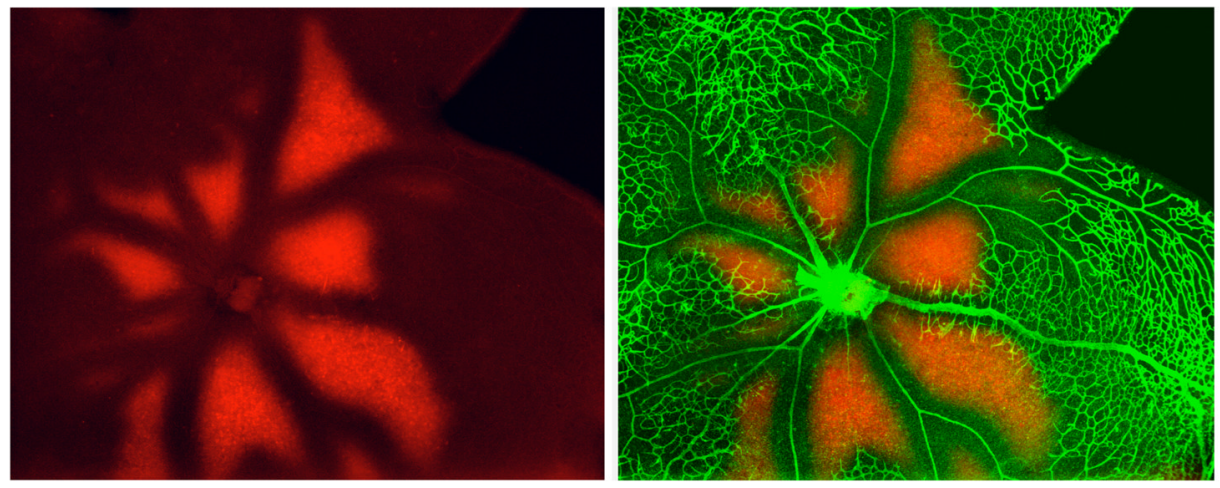

Figure 7.

Flat-mounted retina from P13 mouse (after 75\% O2 from P7 - P12) stained with anti collagen type IV (vessels in green) and anti EF5 (red, hypoxic areas). The animal was injected with the drug EF5 two hours before sacrifice. In hypoxic regions, the drug is reduced and forms permanent protein adducts that can be recognized with an antibody. (Unpublished, courtesy of Marcus Fruttiger). 

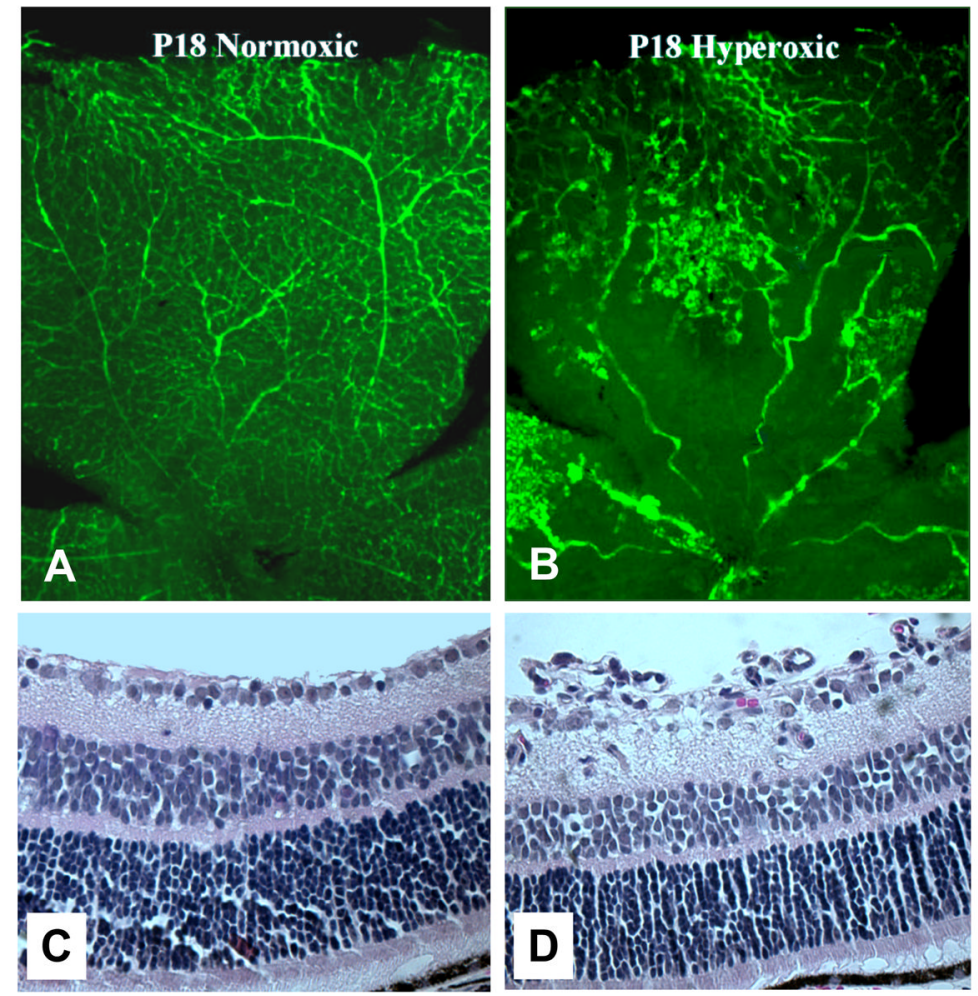

Figure 8.

Panels illustrate retinal neovascularization in the mouse OIR model. Fluorescein-infused retinal flat-mounts (A, B) and H\&E-stained transverse sections $(\mathrm{C}, \mathrm{D})$ of retinas from P18 mice raised in room air (A, C) or treated with hyperoxia between P7 and P12 (B, D). Neovascular tufts can be observed in the retinal mid-periphery at the boundary between vascular and avascular retinal regions. 

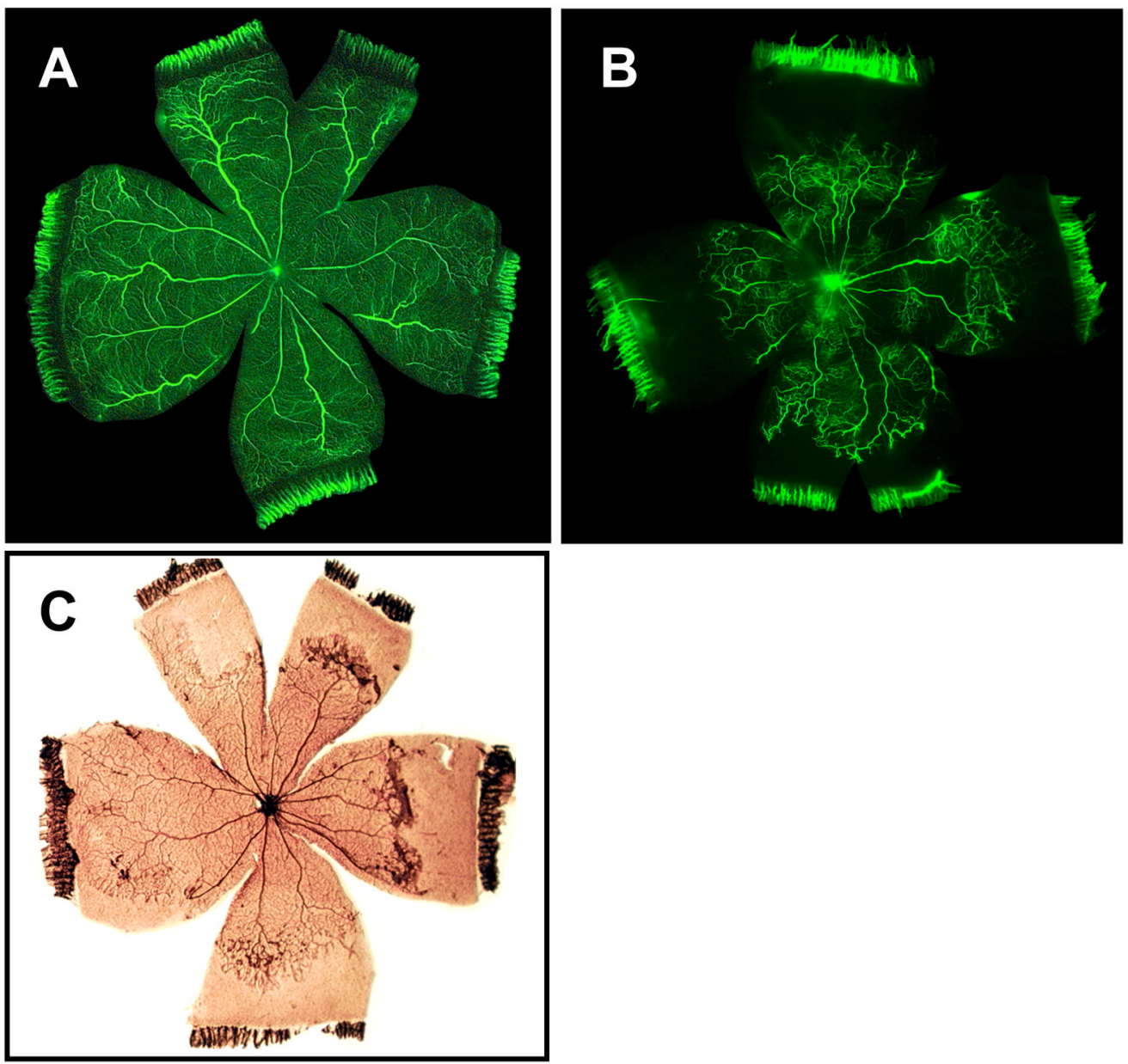

Figure 9.

Panels illustrate retinal neovascularization in the rat OIR model. Fluorescein-infused retinal flat-mounts from room air (A) and OIR (B) treatments show vaso-attenuation of the retinal periphery in the latter. After a brief post-oxygen exposure period in room air, neovascular tufts arise in the mid-periphery as illustrated in ADPase-stained retinal flat mounts (C). 


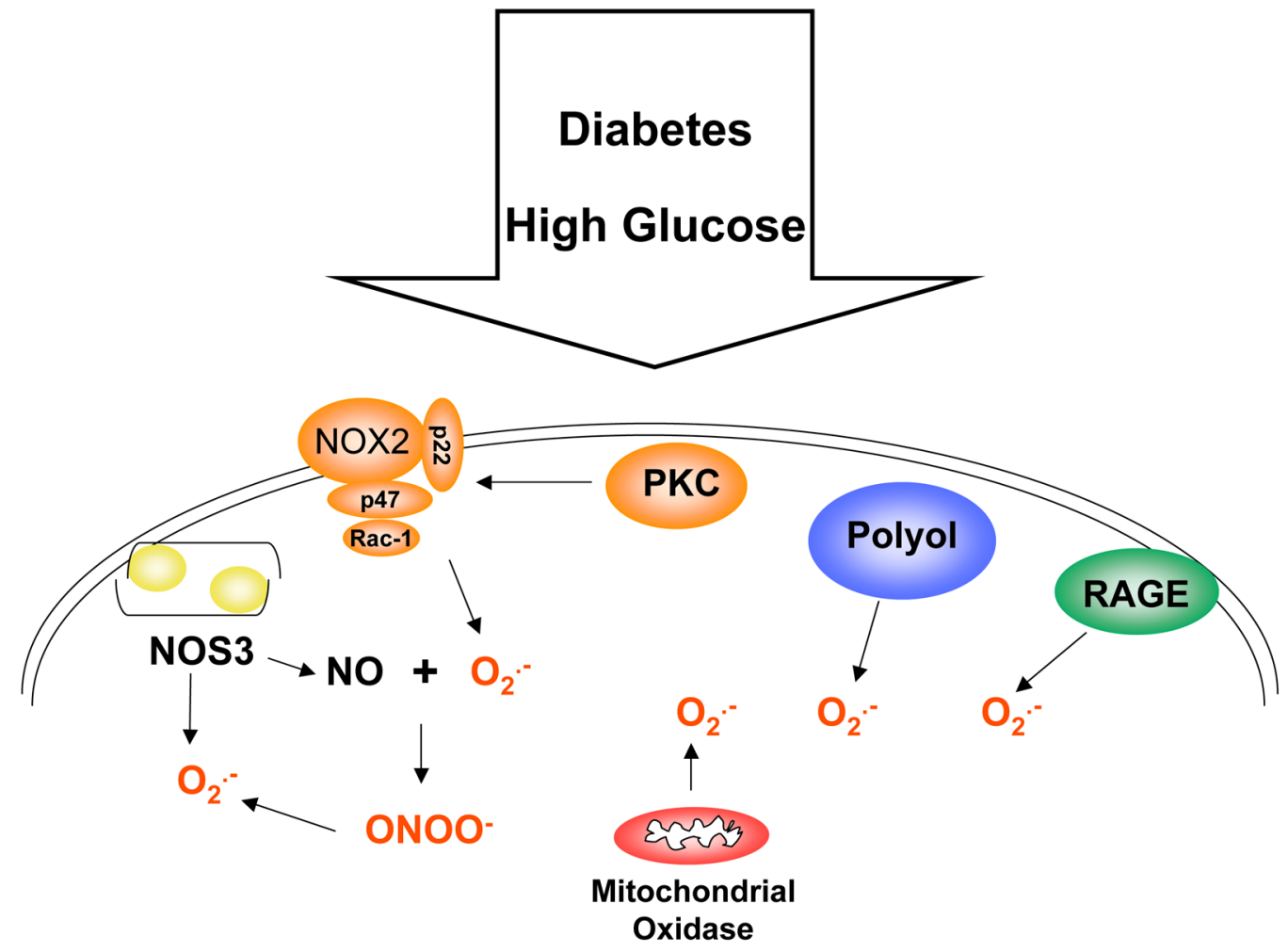

Figure 10.

High glucose treatment of EC leads to superoxide formation, an increase in intracellular calcium and activation of endothelial NOS (NOS3) to form NO, but it also causes NOS3 "uncoupling" to generate superoxide and NO. 


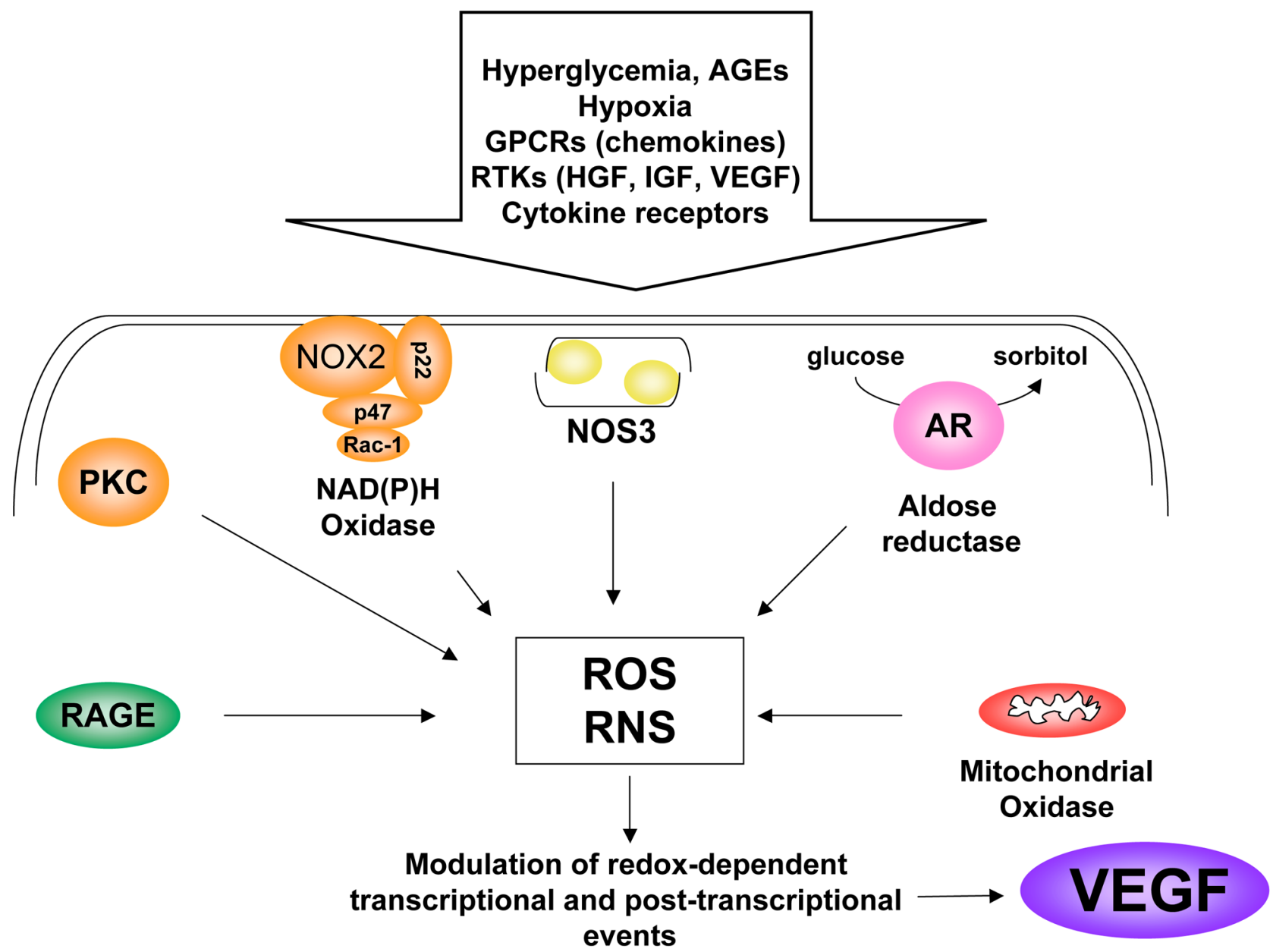

Figure 11.

Numerous factors can contribute to VEGF over-expression in diabetic retinopathy (ROS, reactive oxygen species; RNS, reactive nitrogen species). 


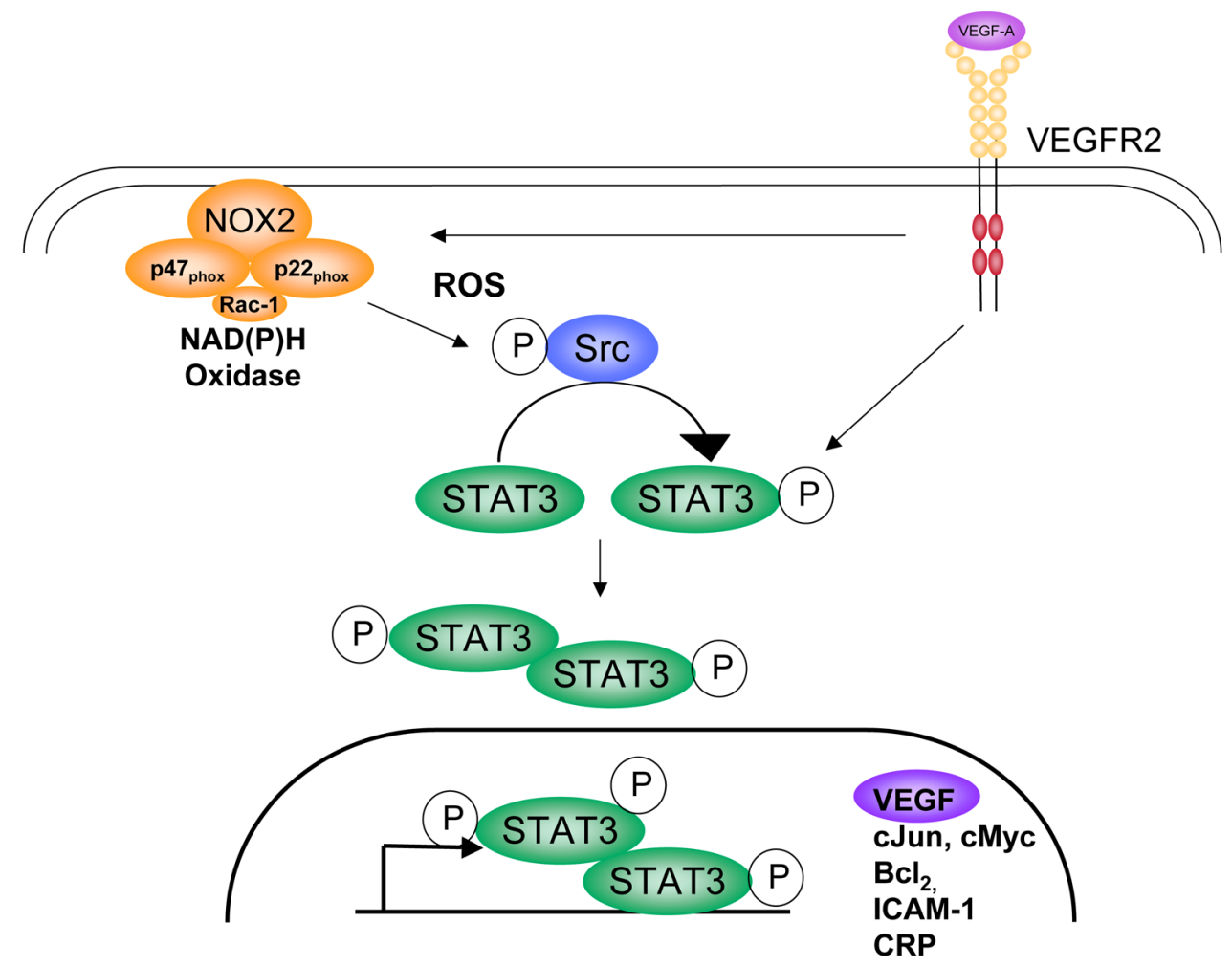

Figure 12.

Proposed role of STAT3 in oxidative stress-induced upregulation of VEGF. Oxidative stress can activate STAT3 by phosphorylation of tyrosine 705 within the activation domain. STAT3 activation may lead to VEGF production in response to both inflammatory and hypoxic stimuli. 


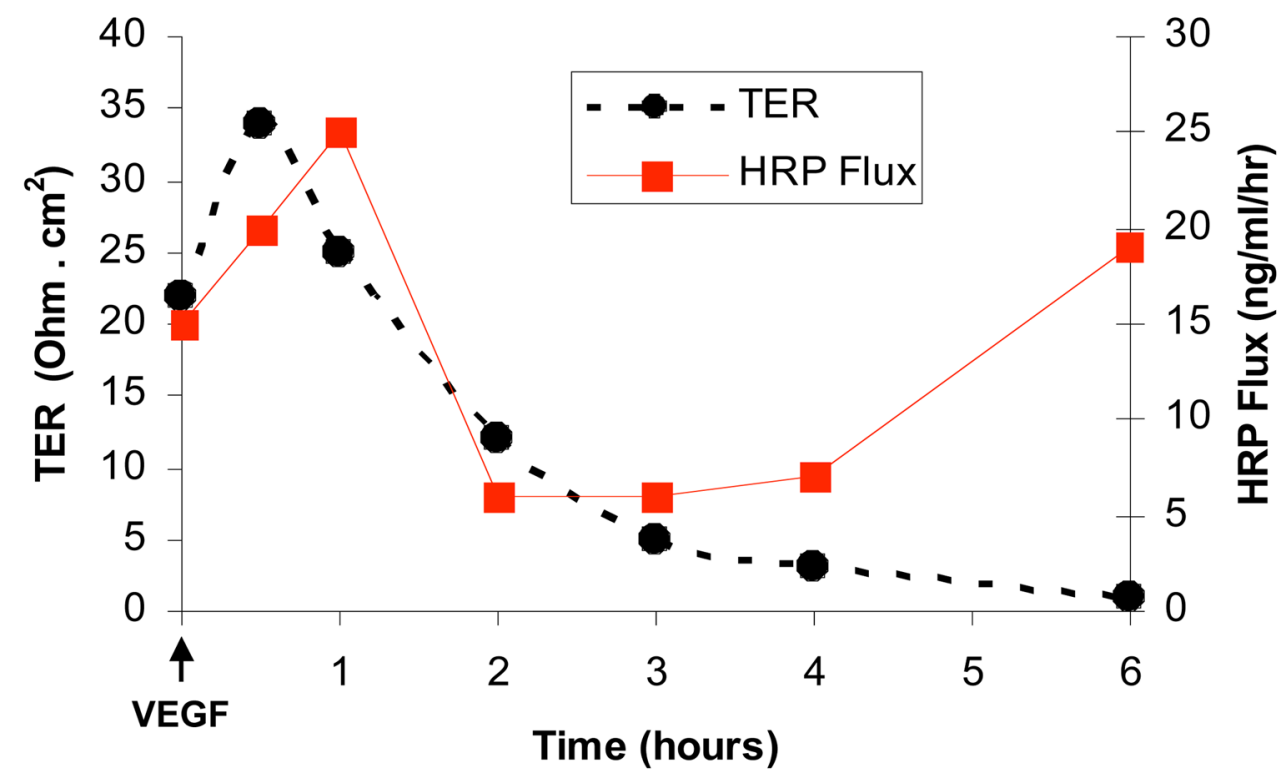

Figure 13.

VEGF stimulation causes a transient increase in transcellular electrical resistance (TER) across the retinal endothelial cell monolayer, which is followed by a gradual decline in TER. The TER increase is associated with a rapid, but transient increase in flux of horseradish peroxidase (HRP), which is followed by a transient recovery of the barrier function and a delayed, but sustained, increase in tracer flux. 

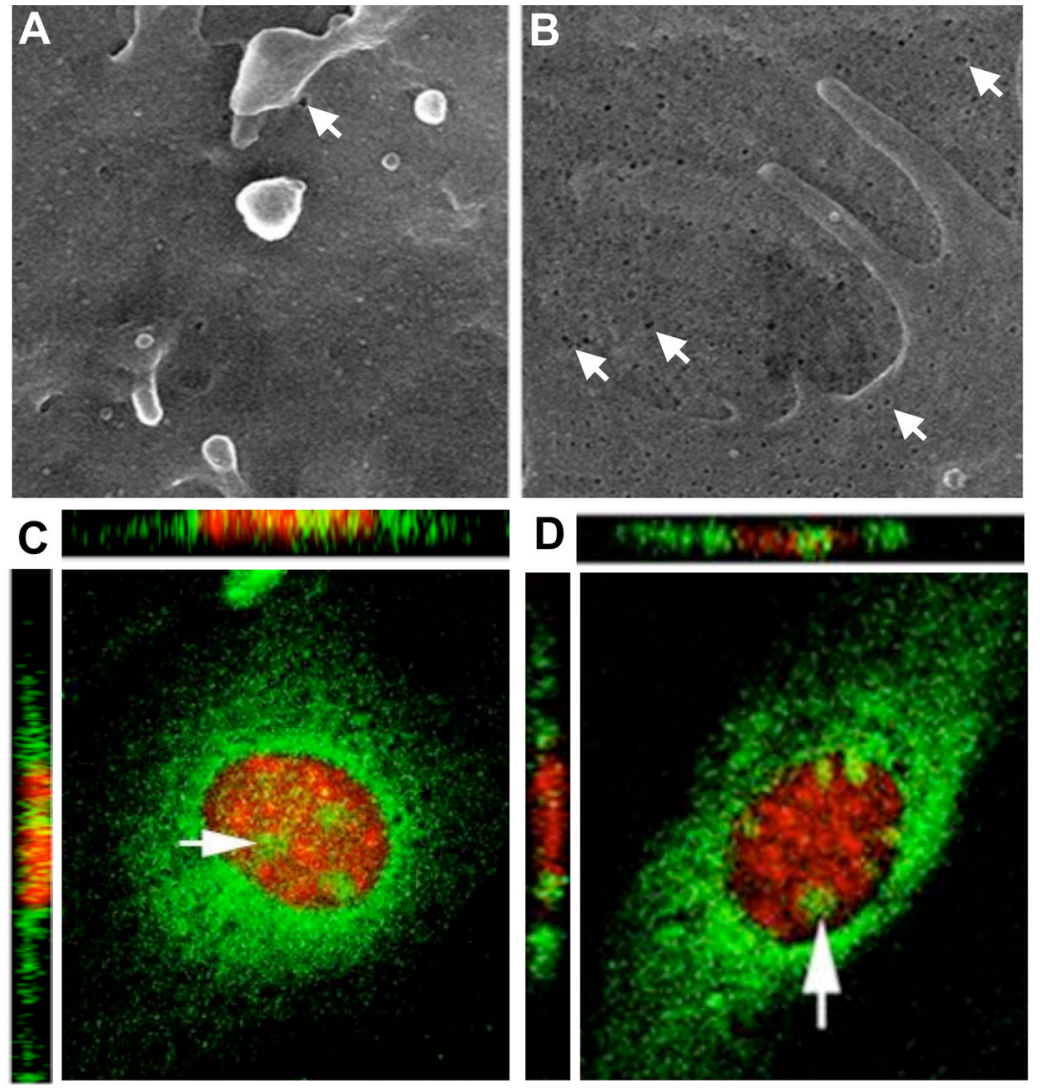

Figure 14.

Retinal endothelial cells exhibit very few plasma membrane caveolae (arrow in A). VEGF treatment induces a significant increase in caveolae formation (arrows in B) and promotes the nuclear translocation of VEGFR2 (C, green label) and NOS3 (D, green label). 

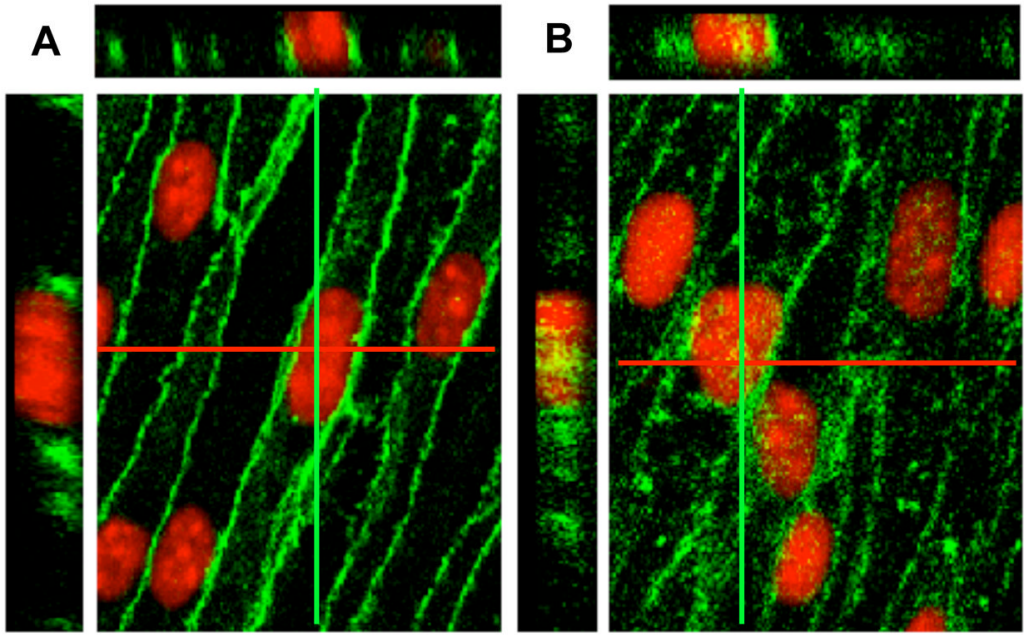

Figure 15.

$\beta$-catenin is localized to cell-cell junctions in untreated retinal endothelial cells (A), but redistributes to the cytosol and nucleus upon VEGF stimulation (B). 


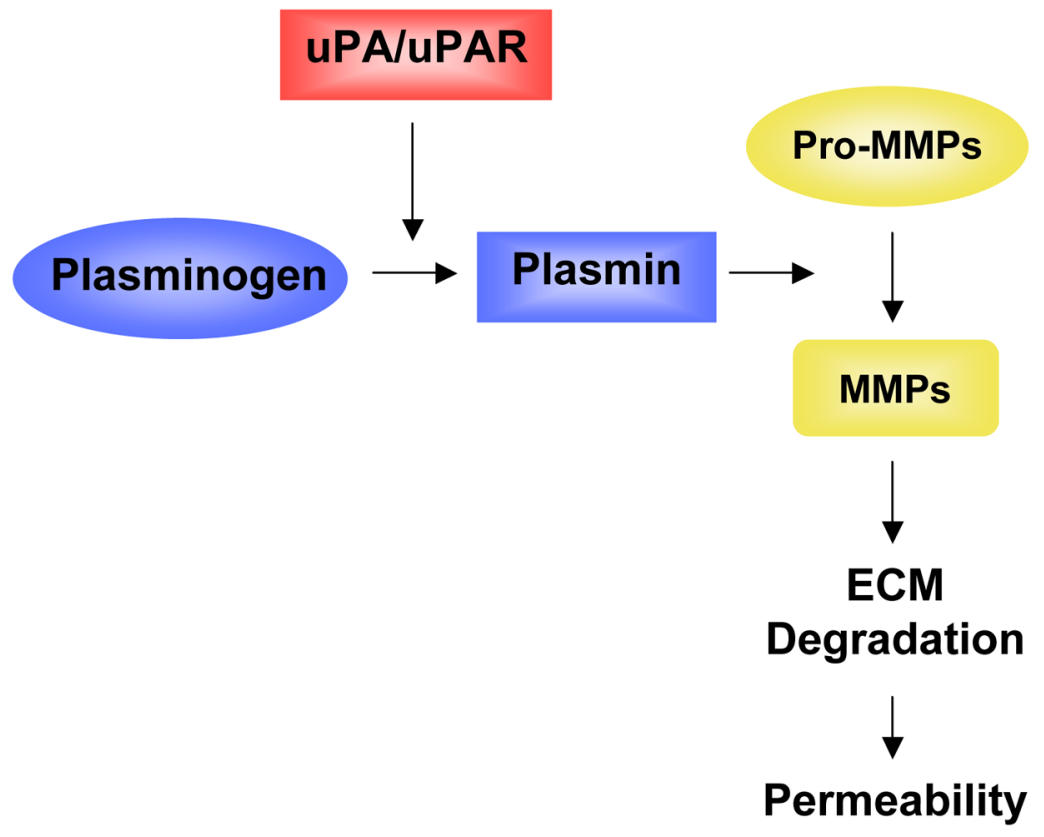

Figure 16.

Expression of UPAR initiates the UPA-mediated proteolytic cascade. MMP activity is associated with ECM degradation, increased vessel permeability and growth factor liberation, all of which promote diabetic vascular pathology. 


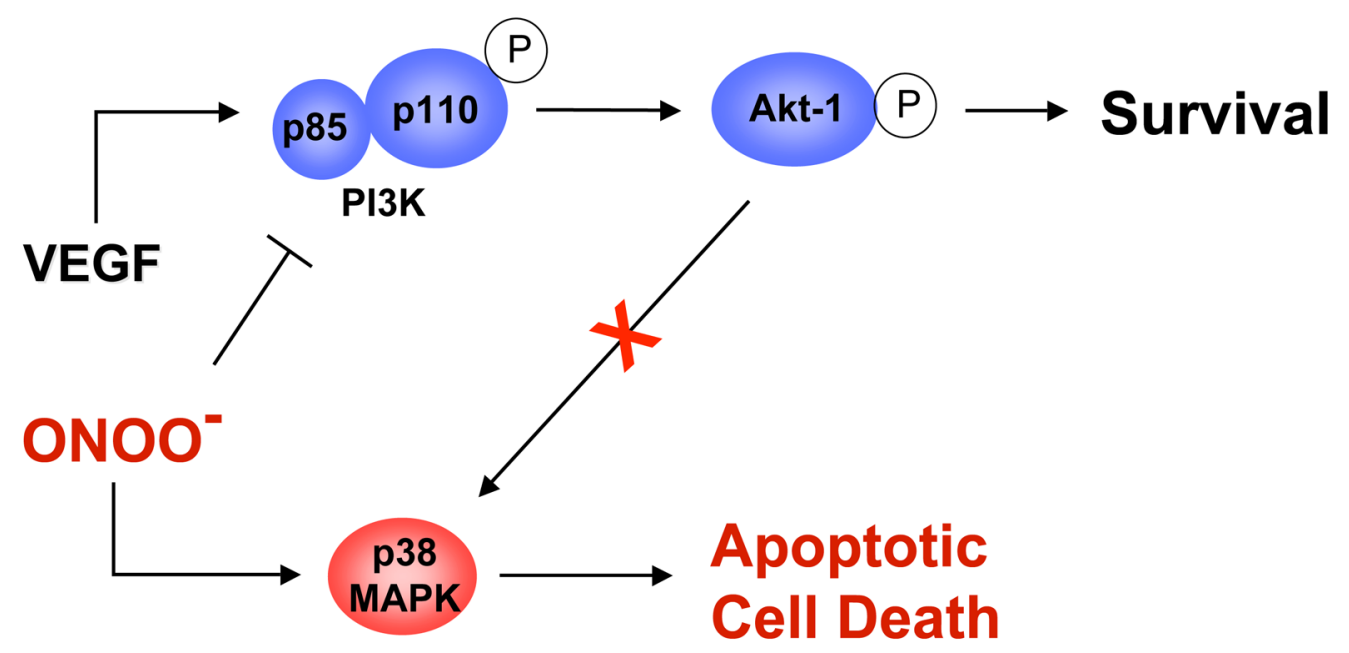

Figure 17.

A schematic representation of the proposed mechanism by which high glucose, via its effect on peroxynitrite, inactivates the VEGF/PI3K/Akt-1 pro-survival pathway and stimulates cell death via activation of p38 MAP kinase pathway. Nitration of PI3K is proposed as a mechanism by which high glucose switches off the VEGF pro-survival pathway and triggers the pro-apoptotic pathway. 


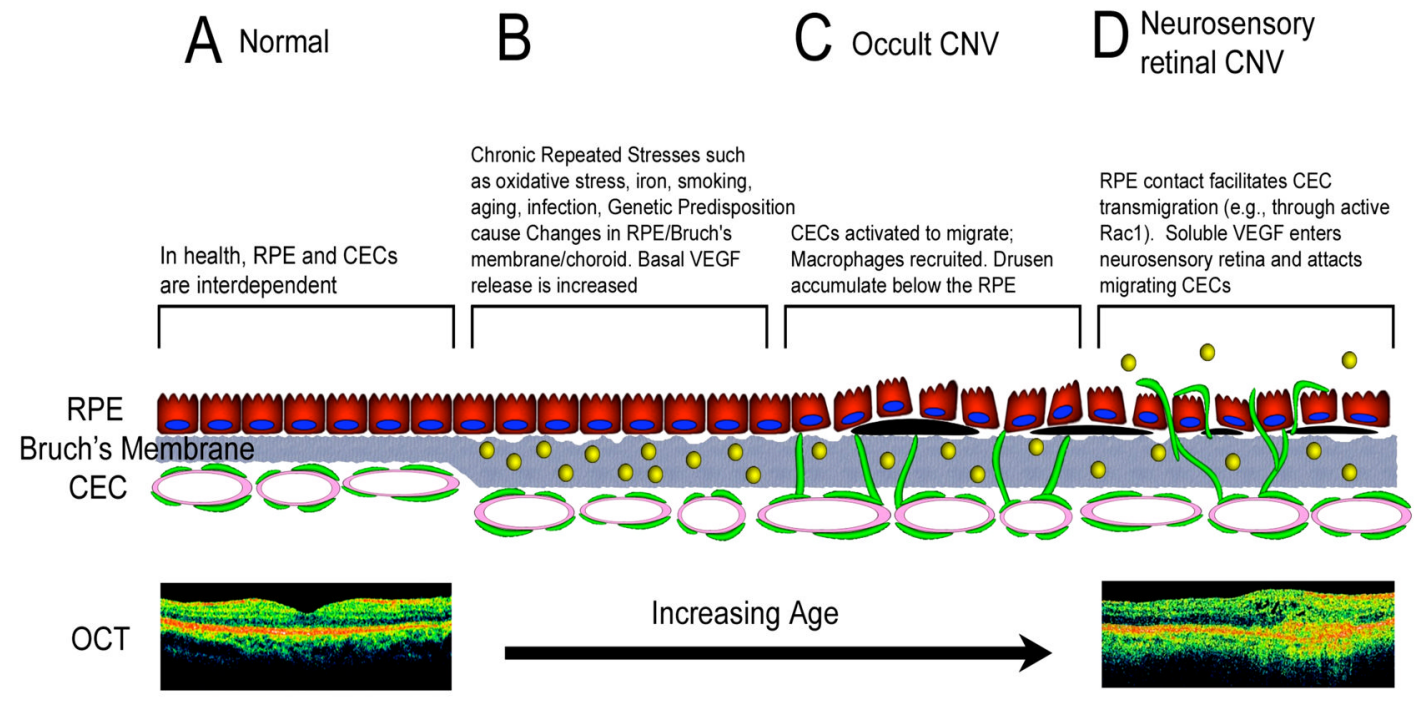

Figure 18.

Diagram illustrating the hypothesis linking VEGF expression, genetic predisposition and changes in the RPE/Bruch's membrane/choroid region with increasing age leading to neovascular AMD and culminating in neurosensory retinal CNV and vision loss. 
Figure 19A.
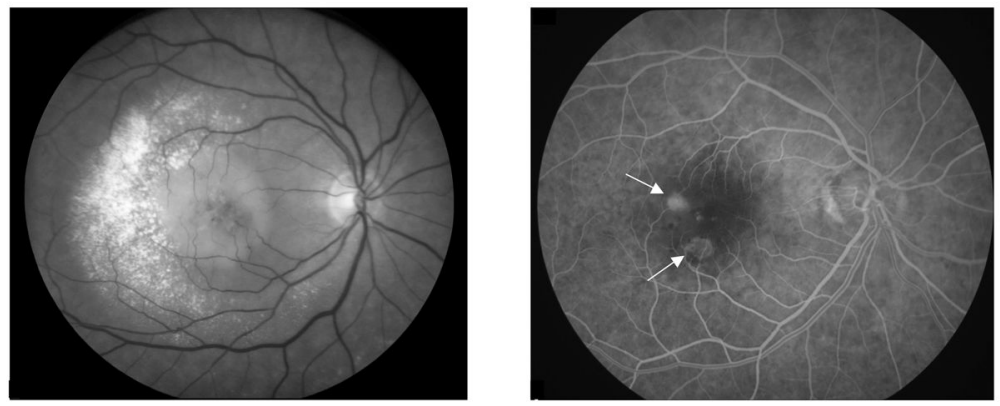

Figure 19B.
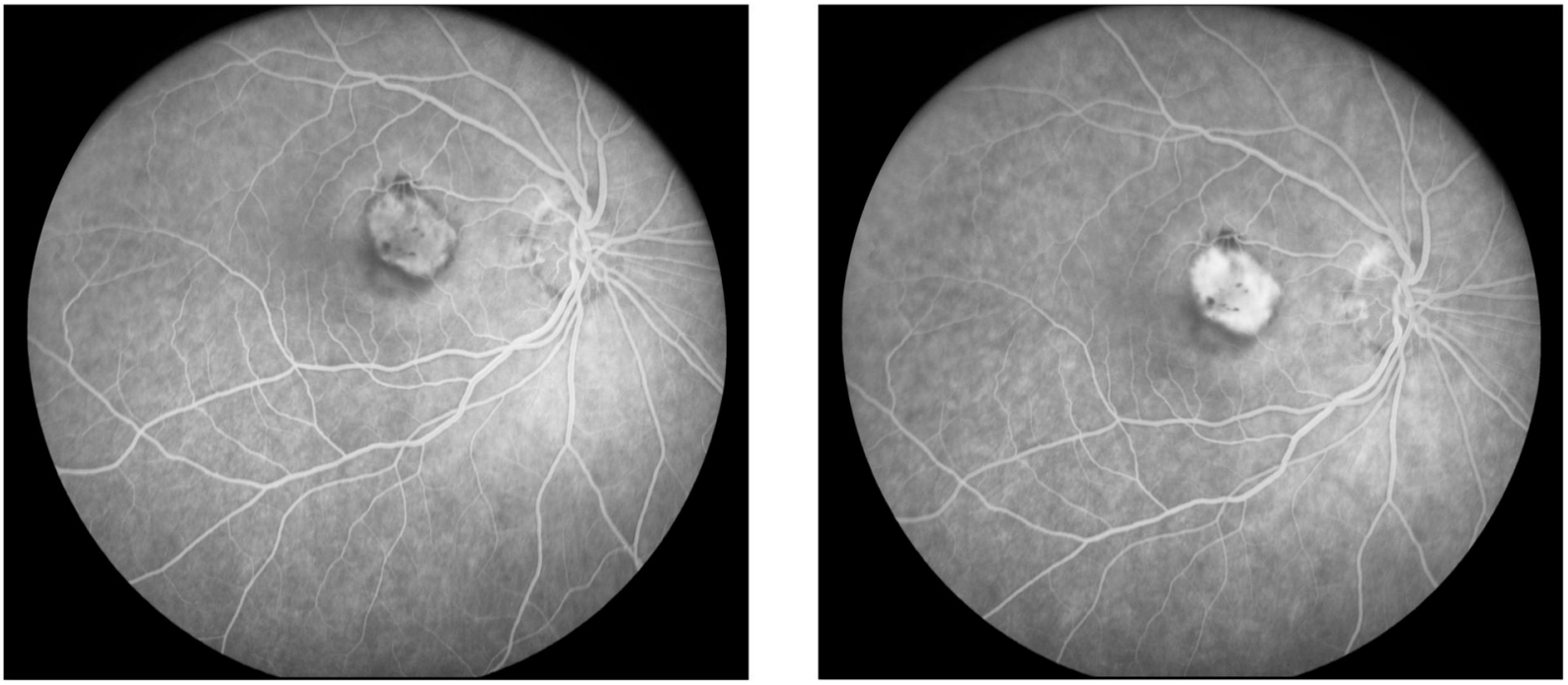

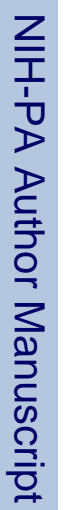

Prog Retin Eye Res. Author manuscript; available in PMC 2013 June 14. 
Figure 19C.
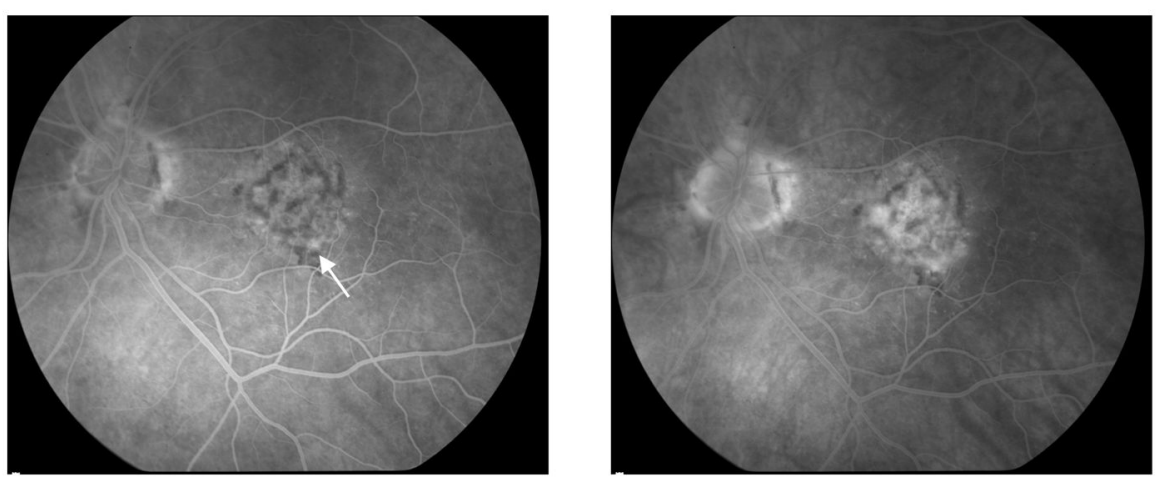

Figure 19.

Figure 19A. Red-free (left) and laminar phase fluorescein angiograms (right) demonstrating retinal vascular abnormalities/anomalous complexes (RVACs; arrows), in which retinovascular circulation feeds deep intra- or subretinal neovascular complexes. There is a pigment epithelial detachment best noted on the red-free image and exudation temporally. Figure 19B. Mid-phase (left) and later phase (right) fluorescein angiograms of mainly classic (neurosensory retinal) $\mathrm{CNV}$.

Figure 19C. Mid-phase (left) and late frame (right) fluorescein angiograms of predominantly occult CNV. There are several hyperfluorescent areas (example, arrow) in late frames showing possible "breakthrough" of CNV into the neurosensory retina. 

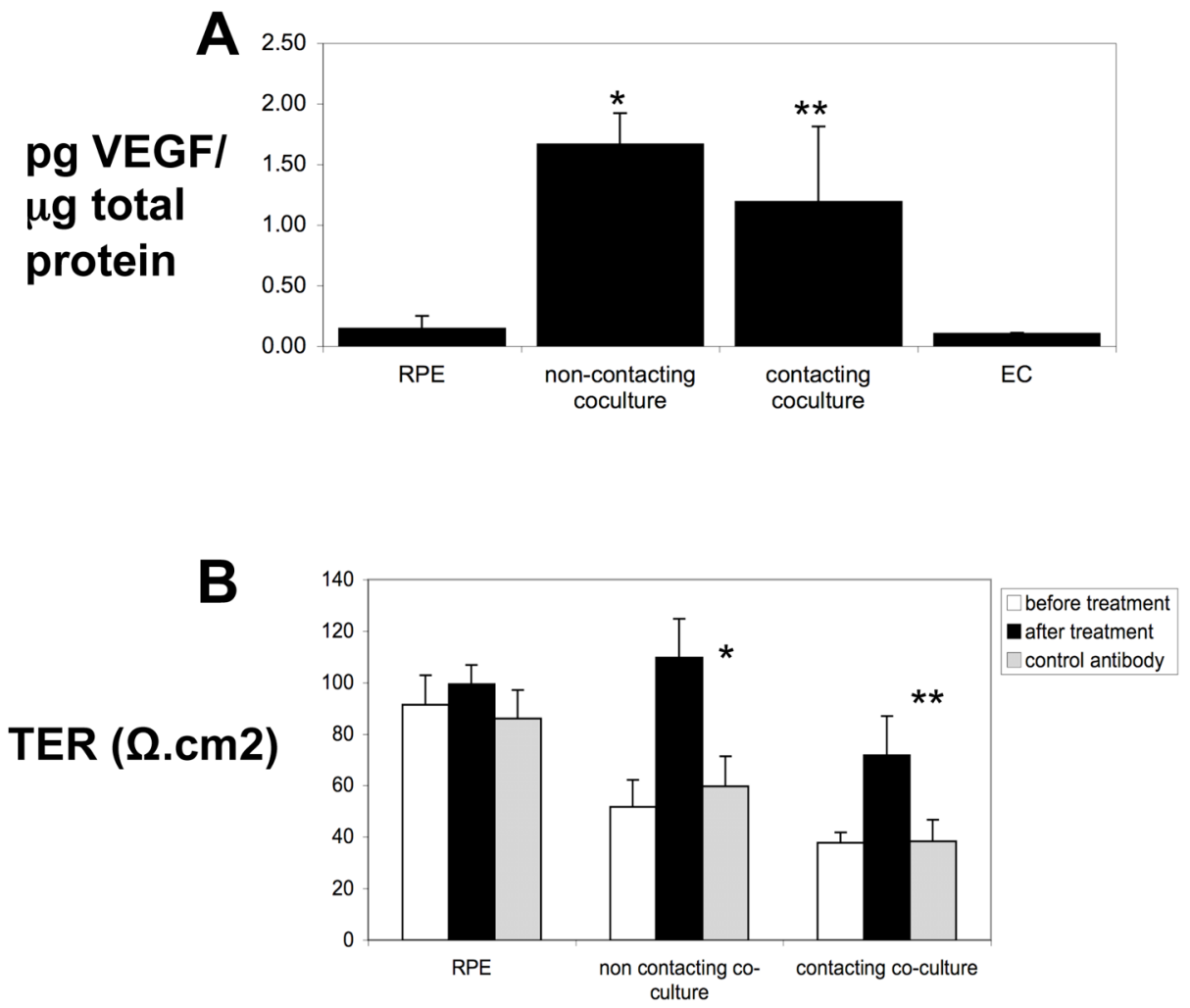

Figure 20.

Figure 20A. Co-culture of bovine retinal microvascular EC and bovine RPE increased the secretion of soluble VEGF into medium ( $*-p=0.008$; ** $-\mathrm{p}<0.05$; one-way ANOVA). B. Co-culture of EC and RPE reduced transepithelial electrical resistance (TER) of RPE through soluble VEGF. Neutralizing antibody to VEGF partially restored TER of RPE in coculture conditions $(*-p<0.001 ; * *-p<0.03$; two-way factorial ANOVA). 


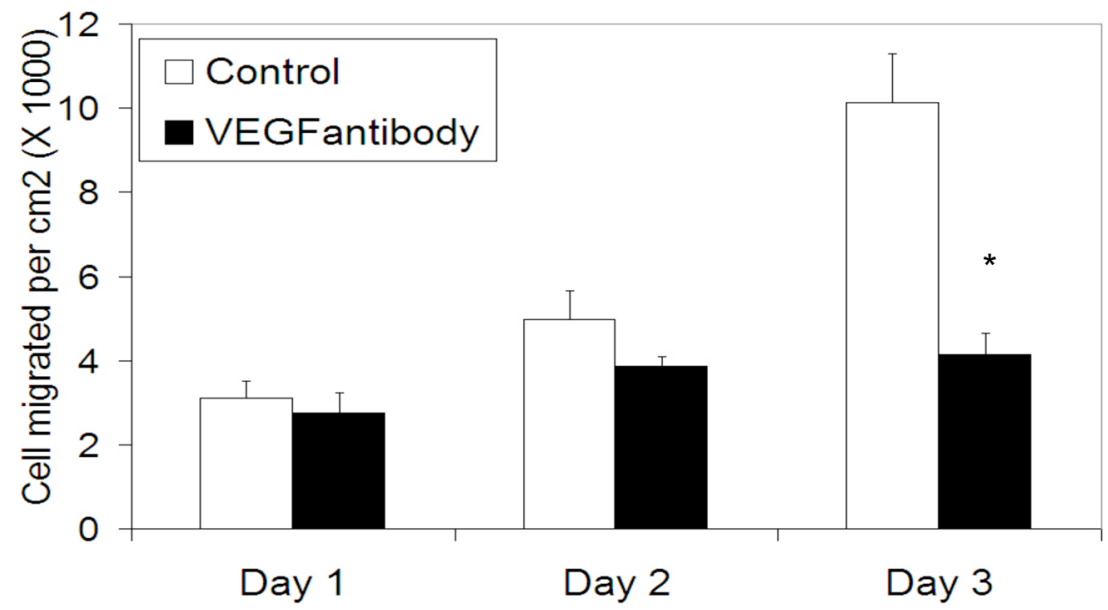

Figure 21.

CECs transmigrate across RPE cells (ARPE-19) over time (white bars). Administation of a neutralizing antibody to VEGF decreases CEC transmigration across ARPE-19 (black bars) $(200 \mathrm{ng} / \mathrm{mL}$ neutralizing antibody to VEGF; * $-\mathrm{p}=0.005$; Student's t-Test). 


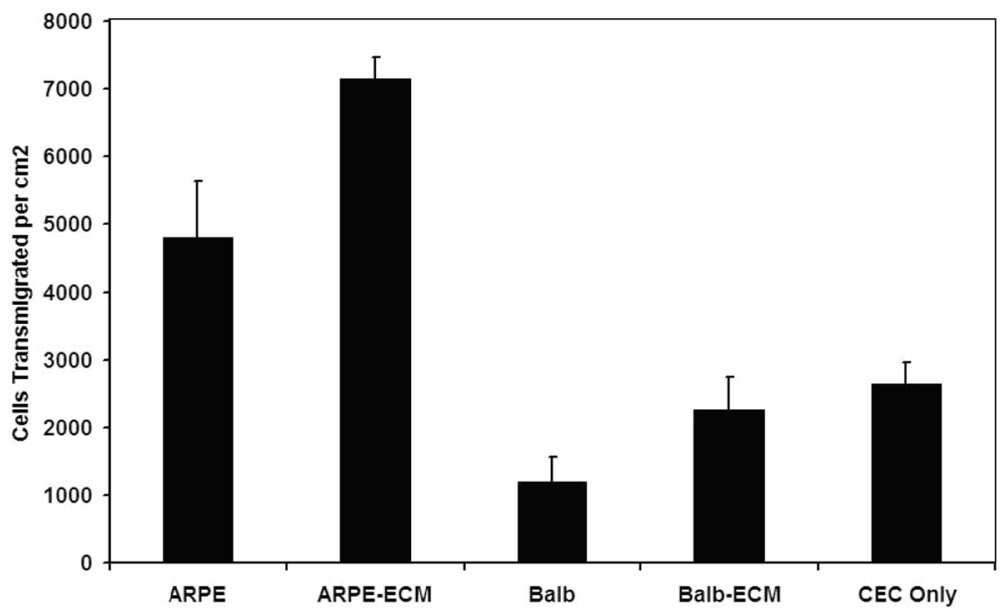

Figure 22.

Enhanced CEC transmigration is observed specifically when cultured in contact with RPE (ARPE-19) and not generally when cultured with a fibroblast cell line (Balb) or fibroblast ECM (Balb-ECM). 


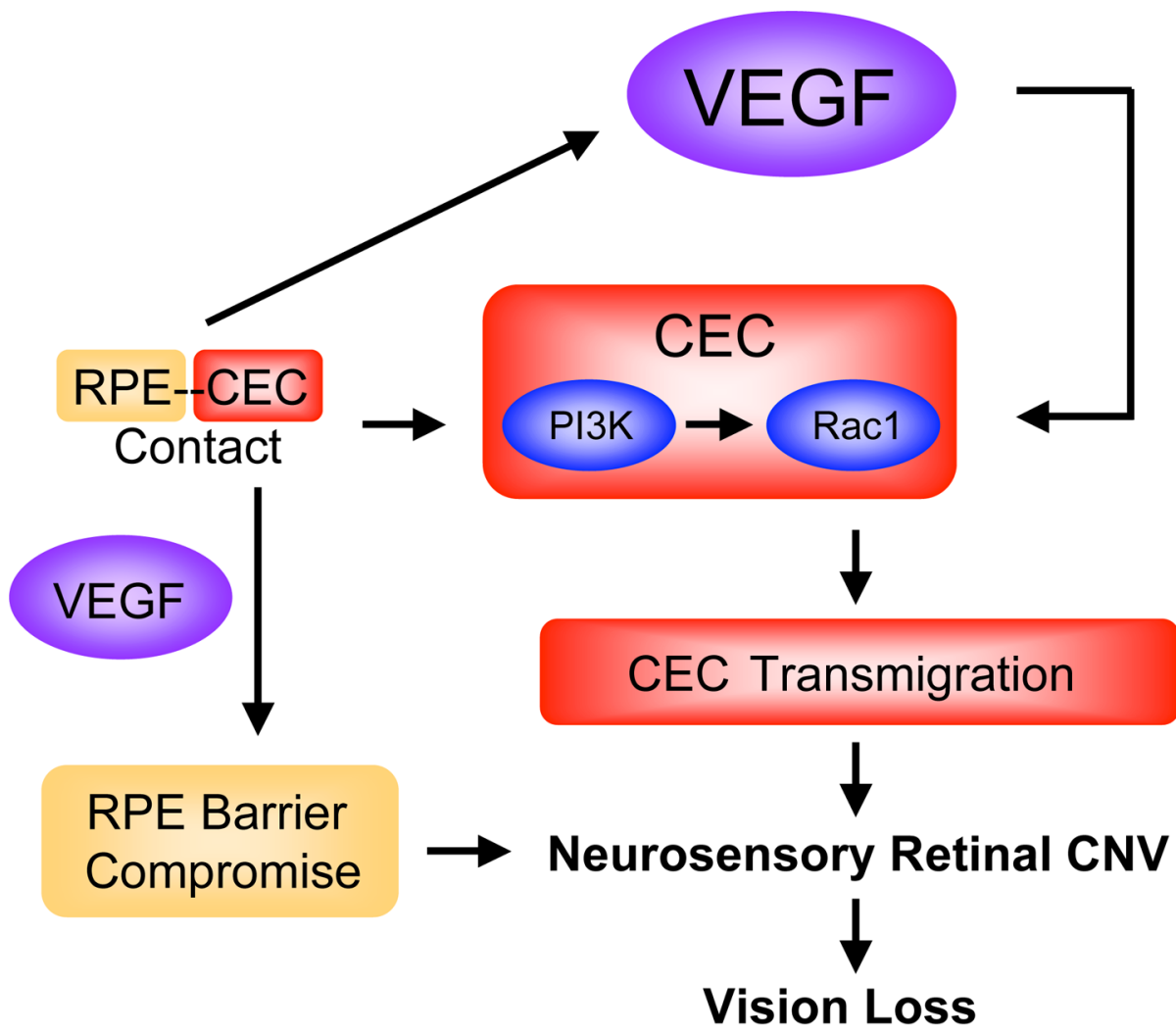

Figure 23.

Contact between RPE and CECs triggers a PI3K-dependent activation of Rac1 in CECs necessary for their transmigration toward the VEGF gradient in the neurosensory retina. VEGF in the neurosensory retina provides a chemotactic gradient for further migration of CECs resulting in "breakthrough" regions on fluorescein angiography, which represent welldefined and sight-threatening CNV. 


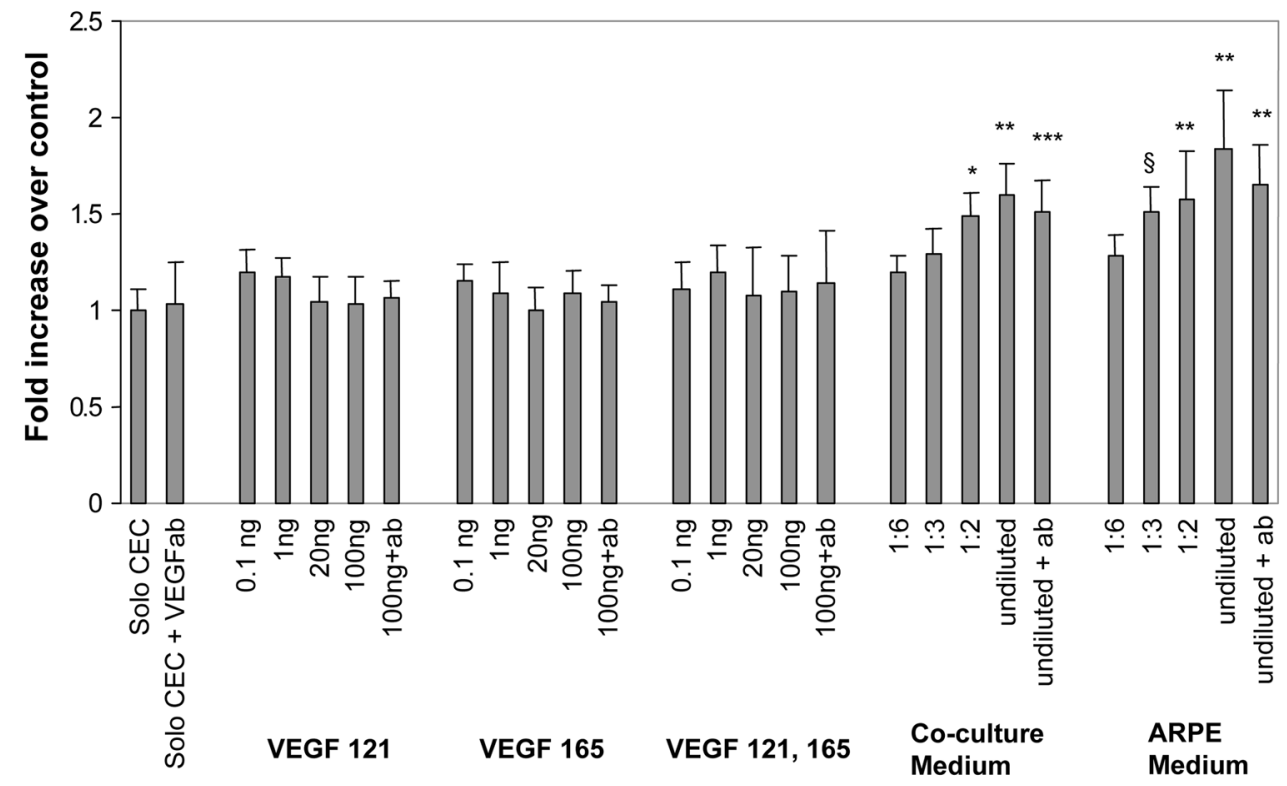

Figure 24.

VEGF did not increase CEC proliferation in vitro, however RPE-CEC co-cultureconditioned medium did. Neutralizing antibody to VEGF did not inhibit CEC proliferation caused by conditioned medium (ANOVA - $\mathrm{p}=0.001 ; *-\mathrm{p}=0.034 ; * *-\mathrm{p}=0.001 ; * * *-\mathrm{p}$ $=0.015 ; \S-\mathrm{p}=0.009 ;$ Student's t-Test with Bonferroni correction, $\mathrm{n}=$ at least 9 ). 